Illinois State University

ISU ReD: Research and eData

Theses and Dissertations

$5-24-2016$

\title{
Split Wounds: Diverging Formations of Trauma in The Diagnostic and Statistical Manual of Mental Disorders V, Girl with the Dragon Tattoo, And the Rat Laughed, and Once Were Warriors
}

Emily R. Johnston

Illinois State University, eronayjohnston@gmail.com

Follow this and additional works at: https://ir.library.illinoisstate.edu/etd

Part of the English Language and Literature Commons, Film and Media Studies Commons, and the Women's Studies Commons

\section{Recommended Citation}

Johnston, Emily R., "Split Wounds: Diverging Formations of Trauma in The Diagnostic and Statistical Manual of Mental Disorders V, Girl with the Dragon Tattoo, And the Rat Laughed, and Once Were Warriors" (2016). Theses and Dissertations. 574.

https://ir.library.illinoisstate.edu/etd/574

This Dissertation is brought to you for free and open access by ISU ReD: Research and eData. It has been accepted for inclusion in Theses and Dissertations by an authorized administrator of ISU ReD: Research and eData. For more information, please contact ISUReD@ilstu.edu. 


\author{
SPLIT WOUNDS: DIVERGING FORMATIONS OF TRAUMA \\ IN THE DIAGNOSTIC AND STATISTICAL MANUAL OF \\ MENTAL DISORDERS V, GIRL WITH THE DRAGON \\ TATTOO, AND THE RAT LAUGHED, \\ AND ONCE WERE WARRIORS
}

Emily Ronay Johnston

228 Pages

Split Wounds interrogates naturalized, normalized trauma wisdom—particularly the individualization and pathologization of sexualized trauma. Drawing on Foucault's concept of discursive formation, explicated in The Archaeology of Knowledge as a set of conditions that enables history, this dissertation elucidates differing discursive formations of trauma in contemporary medical documents, literary texts, and films. The introductory chapter explicates how founding texts in the field of trauma theory construct trauma as a preverbal, psychological experience that can only be represented through fragmented, non-linear, anti-narrative textual strategies. Chapter two exposes such Euro-American modernist ideology in the American Psychiatric Association's clinical definition of posttraumatic stress disorder outlined in The Diagnostic and Statistical Manual of Mental Disorders (DSM-5), which configures trauma as a personal failure to assimilate catastrophic events. Chapter three problematizes the pathologization of trauma survivors, 
illustrating how Stieg Larsson's Girl With the Dragon Tattoo and Niels Arden Oplev's and David Fincher's film adaptations condition us to perceive trauma as a systemic pathology that catalyzes feminist vigilantism. Chapter four demonstrates how collective acts of memorialization transform systemic pathologies that produce sexualized violence, showcasing co-created trauma testimonies in Nava Semel's hybrid Nazi Holocaust novel, And the Rat Laughed. Split Wounds concludes with a fifth chapter that articulates rhetorical strategies for teaching traumatic texts, demonstrating how Alan Duff's Once Were Warriors and Lee Tamahori's film adaptation call upon students to bear witness to the ongoing trauma of internalized oppression rampant in indigenous groups colonized by Europeans.

KEYWORDS: Biomedical, Discourse, Feminism, Gender, Sexualized Violence, Trauma 
SPLIT WOUNDS: DIVERGING FORMATIONS OF TRAUMA

IN THE DIAGNOSTIC AND STATISTICAL MANUAL OF

MENTAL DISORDERS V, GIRL WITH THE DRAGON

TATTOO, AND THE RAT LAUGHED,

AND ONCE WERE WARRIORS

EMILY RONAY JOHNSTON

A Dissertation Submitted in Partial

Fulfillment of the Requirements

for the Degree of

DOCTOR OF PHILOSOPHY

Department of English

ILLINOIS STATE UNIVERSITY

2016 
C 2016 Emily Ronay Johnston 
SPLIT WOUNDS: DIVERGING FORMATIONS OF TRAUMA

IN THE DIAGNOSTIC AND STATISTICAL MANUAL OF

MENTAL DISORDERS V, GIRL WITH THE DRAGON

TATTOO, AND THE RAT LAUGHED,

AND ONCE WERE WARRIORS

EMILY RONAY JOHNSTON

COMMITTEE MEMBERS:

Kirstin Hotelling Zona, Chair

Christopher Breu

Joyce R. Walker

Alison Bailey 


\section{ACKNOWLEDGMENTS}

The writer wishes to acknowledge the many, many people who have helped to make this dissertation possible. Firstly, thank you, Dr. Kirstin Hotelling Zona, my dissertation director, for your steadfast support, compassion, and friendship. At the same time as you remind me that I am infinitely loved, your mentorship also nudges me beyond my comfort zones, toward the heartbreaking, yet necessary writing I know I need to do as a trauma survivor, activist, and teacher-scholar.

Secondly, thank you to my dissertation committee members. Thank you, Dr. Alison Bailey, for pushing my work further than I thought it (or I) could go. Your incisive feedback on my dissertation as well as papers written in your feminist theory course, which planted seeds for this dissertation project, have helped me become a more ethical, articulate feminist. Thank you, Dr. Christopher Breu, for joining the committee and offering generous, much-needed feedback during a time of painful transition. Thank you, Dr. Joyce Walker, for being a personal and professional guide over these last six years.

Thirdly, thank you to other mentors who have guided this dissertation project as well. Thank you, Dr. Rebecca Saunders, for helping to shape the early stages of this project with your comprehensive knowledge of trauma theory/trauma studies. Other past and present mentors who have helped this project evolve include Ricardo Cruz, Dr. Angela Haas, Dr. Julie Jung, Dr. Lee Brasseur, Dr. Kass Fleisher, Dr. Paula Ressler, Dr. Becca Chase, Dr. Paul Ugor, and Dr. Shelly Clevenger, among many other faculty 
members at Illinois State University. Thank you for being a part of this journey with me. Thank you to Pamm Ambrose, Bunmi Adanri, and the rest of the professional staff at the Illinois State Julie N. Visor Academic Center. You have cheered me on this last year, providing much-needed motivation for crossing the finish line.

Fourthly, I want to acknowledge the beloved friends and family members who have loved me through the darkest of times throughout the dissertation process. Mom, Dad, and Alex - my achievements are yours. You have never stopped believing in me, and I hope that I can demonstrate to others the unconditional love with which you have gifted me. Thank you to my peeps: Summer Qabazard, Jessica Young, Dr. Kristi McDuffie, Jeffrey Higgins, Dr. Lasantha Rodrigo, Samantha Case, Christopher Wiman, Nathan Parks, Jeanna Campbell, Stephanie Jones, Laurel Perez, Dr. Barbi SmyserFauble, Michael Wollitz, Dr. Shailen Mishra, Michelle Wright-Dottore, Michelle Grzybowski, Gina Stinnett, and Denise LaGrassa.

Finally, thank you to the Illinois State University Graduate School for awarding me with a generous grant to complete this dissertation. Also, thank you to the editors of Antipodes: A Global Journal of Australian and New Zealand Literature for publishing a previous version of the fifth chapter, an article entitled, "Trauma Theory As Activist Pedagogy: Engaging Studies as Reader-Witnesses of Colonial Trauma in Once Were Warriors.

E. R. J. 


\section{CONTENTS}

Page

ACKNOWLEDGMENTS $\quad$ i

CONTENTS

CHAPTER

I. INTRODUCTION: THE EURO-AMERICANIZATION OF TRAUMA 1

II. A CASE STUDY OF MEDICAL TRAUMA DISCOURSES: DISCURSIVE FORMATIONS OF TRAUMA IN THE DIAGNOSTIC AND STATISTICAL MANUAL OF MENTAL DISORDERS V

DSM: An Epistemo-Politics of Health and Wellness 23

The Social Construction of Medical Knowledge 33

The Social Construction of Mental Illness $\quad 40$

"The medical polarity of the normal and the pathological" 47

Traumatic Exposure $\quad 56$

Traumatic Intrusion $\quad 59$

Traumatic Avoidance $\quad 62$

Traumatic Negativity $\quad 66$

Traumatic Arousal $\quad 74$

Effects of Trauma Symptoms $\quad 76$

Substances and Other Medical Conditions 78

$\begin{array}{ll}\text { Conclusion: Diagnosing Events } & 80\end{array}$

III. A CASE STUDY OF LITERARY AND CINEMATIC TRAUMA DISCOURSES: DISCURSIVE FORMATIONS OF TRAUMA IN GIRL WITH THE DRAGON TATTOO

Trauma: A Systemic Pathology 85

Restaging Trauma and Feminist Vigilantism 98

Challenging Traumatic Truths 114

Conclusion 
IV. A CASE STUDY OF LITERARY TRAUMA DISCOURSES: DISCURSIVE FORMATIONS OF TRAUMA IN AND THE RAT LAUGHED

The Hybridization of Nazi Holocaust Trauma

Collectivizing Traumatic Experience

121

Functionalizing Trauma Responses

Re-localizing Trauma

A Conclusion

V. CO-WITNESSING TRAUMA: BEARING TRAUMATIC WITNESS IN LITERATURE CLASSROOMS

Reading As Witnessing

Listening to the Text

Once Were Warriors: A Context for Understanding

Decolonial Activism in the Classroom

VI. CODA: TRAUMA-A RECONCILIATION 215

REFERENCES 


\section{CHAPTER I}

\section{INTRODUCTION: THE EURO-AMERICANIZATION OF TRAUMA}

Trauma comes from the Greek word for wound, and signifies bodily injury. In seventeenth century medicine when the term was first taken up in English, trauma was used to refer to both "what wounded" and "what cured"-for example, "traumatic balsams" soothed traumas to the skin, such as lacerations (Luckhurst 2). Contemporary medicine, particularly psychiatry, increasingly defines trauma as not only bodily, but also psychic, injury. As Roger Luckhurst explains in The Trauma Question, trauma has come to signify a kind of transmissible rupture — " a piercing or breach of a border that puts inside and outside into a strange communion [...] [that] leaks between mental and physical symptoms $[\ldots]$ between patients and doctors $[\ldots]$ and between victims and their listeners or viewers" (ibid). Trauma, that is, can be understood as a contagion, a wound that travels, dissolving boundaries between body and mind, patient and doctor, survivor and witness.

Trauma can also be understood as a breakage. Theories and clinical studies of trauma suggest that the traumatized psyche cannot process what is happening (a traumatic event) while it is happening, and stores the experience outside of consciousness. The aftermath of traumatic events, then — what psychiatry configures as the trauma (e.g., posttraumatic stress disorder) — is marked by a compulsive, repetitive return to the triggering event in an effort to master the traumatic memory - a compulsion that can 
never be satiated. The aftermath of traumatic events can thereby compound suffering and impede memory, as is evident in the plight of Nazi Holocaust survivors who grapple with having lived through World War II while others perished. As Cathy Caruth explicates in her landmark collection, Trauma: Explorations in Memory, trauma altogether resists static conceptualizations; it is an inevitable impossibility, as "the traumatized [...] carry an impossible history within them, or they become themselves the symptom of a history that they cannot entirely possess" (5). Traumatic events exceed the bounds of time and space, possessing survivors and returning them to the horror against their will in nightmares, flashbacks, and other forms of psychic intrusion.

Theories of trauma, especially those exploring how historical events like World War II live on through survivors and future generations, have long interested scholars and practitioners in the social sciences and more recently, in the humanities. In addition to circulating in different disciplinary and clinical settings, fascination with and theorization of trauma also increasingly occupies the social imaginary. The proliferation of news broadcasts, television shows, films, and other forms of mass media representing war, natural disasters, terrorism, bullying, domestic violence, sexual sadism, and other catastrophes evidences such a turn — as does the American Psychological Association's (APA) recent addition of a section on "Trauma- and Stressor-Related Disorders" in the current (fifth) edition of The Diagnostic and Statistical Manual of Mental Disorders (DSM-5), widely considered psychiatry's bible. The Task Force and Work Groups that collectively revised DSM between its fourth and fifth editions, comprised of worldrenowned clinicians and medical researchers, describe trauma as "increasingly at the center of public as well as professional discussion" ("Posttraumatic Stress Disorder"). 
Trauma has come to not only blur boundaries between psyche and body, patient and doctor, survivor and witness, it has also permeated boundaries demarcating public and professional, interpersonal and institutional, local and global.

While trauma is a fundamentally social, cultural, historical phenomenon, the very inclusion of posttraumatic stress disorder (PTSD) in DSM-5, as well as in its previous third and fourth editions, implies that trauma is a personal pathology that can (and should) be clinically treated. The very concept of mental disorder reveals such a politics of mental health. The APA claims that any diagnosis listed in the DSM is a "mental disorder," and that establishing "consistent, reliable diagnoses" for such disorders, the manual's stated aim, happens interpersonally, between doctor and patient (“American Psychiatric Association"). In order for clinicians to diagnose someone with a DSM disorder, the patient must meet diagnostic criteria listed for that specific disorder, in addition to the manual's general definition of mental disorder: "A syndrome characterized by clinically significant disturbance in an individual's cognition, emotion regulation, or behavior that reflects a dysfunction in the psychological, biological, or developmental processes underlying mental functioning" (emphasis added, DSM-5 20). From this definition, it follows that developing a mental disorder (e.g., PTSD) stems from a mental, rather than a sociocultural or other environmental, dysfunction. Such an assumption about the origins of mental illness pathologizes the person already suffering, locating the source of the problem to be treated (the dysfunction) within singular patients rather than in larger contexts and conditions that produce suffering. Given the absence of precise diagnostic tools for assessing psychological pathology, such as X-rays or blood tests, clinicians diagnose mental illness by way of what is known as a clinical case 
formulation: "a careful clinical history and concise summary of the social, psychological, and biological factors that may have contributed to developing a given mental disorder [...] [that] use[s] the available contextual and diagnostic information in developing a comprehensive treatment plan" (DSM-5 19). The clinical case formulation includes a "diagnostic interview," a 30-minute session in which the clinician asks the patient a series of questions to assess the presence and degree of "signs that indicate the dysfunction of neural circuits" (Nussbaum 5). Upon making a diagnosis, the clinician develops a treatment plan, which often includes ongoing care (e.g, medications, regular therapy sessions). As this protocol suggests, treating so-called "mental disorders" starts and ends with the individual. It assumes the location of illness or disease within a patient; the diagnostic interview facilitates diagnosis, and diagnosis facilitates treatment.

The naturalized belief that trauma is a personal pathology requiring interpersonal (clinical) intervention occludes trauma's aggregate, systemic situatedness, as well as the possibility that the term "trauma" may not effectively, accurately, or appropriately describe catastrophic experience across time and space. Trauma theory itself emerges from a Western biomedical model that prizes technoscientific innovations and commodifies health, and it developed in relation to clinical studies of survivor groups in Europe and the United States, especially war veterans and abused women and children. As a result of these studies, the APA added the category of PTSD to its DSM classification of mental disorders in 1980 (DSM-III), legitimizing traumatic stress as a medical condition necessitating psychiatric intervention.

Following psychiatric studies of PTSD, literary and cultural studies, among other branches of the humanities, took up the study of traumatic injury in relation to testimony, 
literature, and history of the Nazi Holocaust, theorizing trauma as both a psychological and a collective experience. As Jeffrey C. Alexander explains in "Toward A Theory of Cultural Trauma," mass traumas like the Nazi Holocaust signify "a horrendous event that leaves indelible marks upon group consciousness, marking their memories forever and changing their identity in fundamental and irreversible ways" (1). Across different disciplinary settings that engage with trauma theory, the study of trauma centers on the assumption that certain "horrendous events" produce shock, flashbacks, denial, insomnia, headaches, chronic pain, and other symptoms that interfere with one's ability to function in daily life —-from meeting basic needs for food, clothing and shelter, to fulfilling social roles and relating with others. However, as more recent trauma theory scholarship suggests, no reaction to or intervention into horrendous events is invariably necessary, appropriate, shared, or even culturally considered "pathological;" all possible responses to such events must be considered as potentially functional and therapeutic. There are, as this dissertation explicates, endless innovative ways to cope with what is happening right now.

The assumption that so-called "traumatic" events produce (or reveal) pathology, and moreover, the universalization of this assumption across diverse settings in which such events occur, is rooted in Freudian psychoanalysis, which largely functions as the theoretical basis of the field of trauma theory. Put differently, globalized conceptions of trauma are rooted in Euro-American ideologies, including the notion that "mind" is distinct/can be separated from "body;" that psychic stress can and should be treated at the level of the individual; that the psychosomatic symptoms associated with traumatic stress are dysfunctional — that is, that they indicate pathology; and that these symptoms are 
produced by singular events that are "one-off experience[s] safely in the past" (Watters 86). Such naturalized trauma ideologies configure the subject of trauma, the survivor, as "victim."

Psychoanalytic understandings of trauma tend to pathologize survivors of socalled interpersonal traumas in particular (e.g., domestic violence, sexual harassment, rape) as suffering from internal abnormalities that prevent them from avoiding or moving on from traumatic events, including low self-esteem, traumatic stress resulting from childhood abuse, substance abuse, and/or other alleged dysfunctionalities. While many adult victims of intimate partner and sexual abuse do contend with personal histories of violence and fractured self-esteem, pathologizing their trauma responses obscures, even erases altogether, the systemic roots of the violence that may have initially traumatized them. White misogynist systems of power and control produce paradigms around sexuality, gender, family structure, age, religious beliefs and practices, and other cultural constructs that render non-white, female bodies imminently subject to the wills of privileged men.

Psychoanalytic concepts foundational to the study of trauma illustrate Western ideological attachments: dissociation, a detachment or separation from reality; traumatic reenactment, or the repetition compulsion, in which someone repeats difficult or distressing behaviors, reenacting a traumatic event over and over again, or putting oneself in situations where the traumatic event is likely to recur; the unconscious, where painful memories are stored in the brain; and the talking cure, a therapeutic intervention for tapping into the unconscious and bringing painful memories into the open so that they can be dealt with and moved through, enabling the survivor to regain some sense of 
normalcy after the traumatic event(s) (Ringel \& Brandell). The Yale School of trauma theorists and its followers, ${ }^{1}$ widely considered the founding figures of trauma theory in the humanities, build their theories of trauma through these and other psychoanalytic concepts. In grounding their theorizations of trauma in Freudian psychoanalysis, which Freud himself developed through analyzing the experiences of white middle- and upperclass women, the center-staged Yale School (inadvertently) renders invisible nonWestern and minority experiences of, responses to, and strategies of healing from catastrophe, universalizing their theories as applicable to all traumatic experience.

Naturalizing and universalizing Western psychoanalytic conceptions of trauma (bio)medicalizes trauma and causes harm. As Ethan Watters cautions in Crazy Like Us: The Globalization of the American Psyche, when we (Euro-Americans) assume the universality of what the human mind is, we stamp out "the diversity of different cultural understandings of mental health and illness [...] at our own peril" (7). In the case of PTSD in particular, Watters explains, Western understandings of and treatments for traumatic stress "may be poised to spin" the very cycles of violence that incite catastrophic events "back into motion" (125). Trauma, that is, traumatizes.

Through a case study of relief aid in Sri Lanka in the wake of its 2004 tsunami, Watters finds that Western trauma counselors flocking to Sri Lanka effectively replicated settler colonialism in their mission to mediate the alleged psychological effects of this disaster. They believed that their resources for coping with the tsunami's aftermath were

\footnotetext{
${ }^{1}$ Cathy Caruth: Trauma: Explorations in Memory; Unclaimed Experience: Trauma, Narrative and History; Literature in the Ashes of History; and, Listening to Trauma: Conversations with Leaders in the Theory and Treatment of Catastrophic Experience. Shoshana Felman: Testimony: Crises of Witnessing in Literature, Psychoanalysis and History. Dori Laub: co-author of Testimony with Felman. Judith Herman, M.D.: Trauma and Recovery: The Aftermath of Violence-From Domestic Abuse to Political Terror. Bessel A. van der Kolk, M.D.: Traumatic Stress: The Effects of Overwhelming Experience on Mind, Body and Society; and, The Body Keeps the Score: Brain, Mind and Body in the Healing of Trauma.
} 
superior to those of the Sri Lankan people. Moreover, they assumed that the tsunami would, in fact, produce widespread PTSD among the Sri Lankan population, overlooking other responses, such as the possibility that "[ $\mathrm{t}]$ rauma reactions aren't automatic physiological reactions inside the brain [...] but rather cultural communications [with] nuance and meaning that can be misinterpreted or overlooked unless observers have a deep understanding of the culture at hand" (76-77). In addition, trauma relief counselors ignored the possibility that "because of their intense familiarity with poverty, hardship, and war, [Sri Lankans] had evolved a culture better able to integrate and give meaning to terrible events" (88). Where trauma survivors in Euro-American settings, who are medically insured and/or who can otherwise afford psychiatric treatment, might be conditioned to seek out individual counseling to process catastrophic events, many Sri Lankans experienced such interpersonal interventions as detrimental, insofar as they interfered with social roles and duties. Yet the imposition of Western PTSD treatments on the Sri Lankan population (e.g., art therapy as a means of accessing and mastering traumatic memories, counseling centered on airing personal emotions, and even handing out pharmaceutical medications) bypassed altogether Sri Lankan understandings of the tsunami "in terms of the damage it did to social relationships" (91). In naturalized (Western) trauma wisdom, there is lip-service paid to, but little action reflecting, the recognition of catastrophe as always-already cultural — at once located in and fracturing the bonds of community. Without such recognition, traumatologists risk glossing over crucial cultural differences, at best, and perpetuating the very conditions that impel violence and suffering, at worst. This dissertation recognizes—sees again — trauma as irreducibly cultural. 
PTSD is arguably among the most globally-recognized terms for describing the psychological aftermath of catastrophic events. As Watters puts it, PTSD has become "the international lingua franca of human suffering" (71). A category like PTSD has helped people make sense of unbearable suffering, dispelling the shame that can surround that suffering, and making professional interventions (e.g., psychotherapy, pharmaceutical medications) both possible and accessible.

However, as with any category, naturalizing and normalizing PTSD as an alwaysalready byproduct of traumatic events globally can erase crucial differences across different experiences of catastrophe. To define PTSD as a psychic wound, brought about by what traumatologist Judith Herman, M.D. describes as an "extraordinary" event that "overwhelm[s] the ordinary human adaptations to life," is to assume that what is deemed "ordinary" in one setting - and moreover, how humans adapt to and with their environsis basically the same everywhere (33). PTSD universalizes Western conceptions of "ordinary" and "adaptation," when in reality, they are highly divergent. For example, while the September 11, 2001 suicide attacks on the World Trade Center complex in New York City, and the November 13, 2015 suicide bombers in Paris were broadcast globally as catastrophic - that is, out of the ordinary — terrorism is a daily reality in other parts of the world, such as Turkey and Beirut, and may go underreported or unreported altogether. People living in these global settings may be highly adept at adapting to catastrophe; but the daily-ness of terrorism there does not mean it is any less traumatic - that is, any more “ordinary” than 9-11, the 2015 Paris Attack, or other disproportionately publicized terrorist incidents. Moreover, adaptations to such events considered "ordinary" in one setting — visiting a therapist, telling personal testimonies on live news, creating public 
memorials for people injured or killed — may seem quite strange, even inappropriate, in another. Even when there may be global consensus that an event or condition is, indeed, extraordinary, ways of responding to and integrating that event/condition into everyday life vary widely.

Current trauma scholarship highlights gaps between universalized theories and embodied experiences of catastrophe. Jane Kilby, co-editor of The Future of Memory, writes that while trauma is "a global phenomenon [...] something experienced by the world and by any number of people and communities," it also happens "under a range of conditions and according to an array of cultural, historical, political, and social logics" (181). Given trauma's simultaneous local/diverse and global/universal dimensions, we need flexible trauma frameworks and practices - ones that emerge from and account for localized understandings of and responses to suffering, rather than ones imposed by Western (bio)medical doctrine and/or analyses of Euro-American traumas exclusively.

The field of trauma theory is fraught with Euro-Americanism. In Postcolonial Witnessing: Trauma Out of Bounds, Stef Craps claims that the field fails "to live up to [its] promise of cross-cultural engagement" as it marginalizes non-Western and minority traumas, universalizes Western psychoanalytic conceptions of trauma and recovery, and normalizes "a modernist aesthetic of fragmentation and aporia" as the sole means of textually representing traumatic suffering (2). Craps, and others reflecting on the Western foundations of trauma theory and constructing new directions for the field, ${ }^{2}$ argue that in

\footnotetext{
2 Jeffrey C. Alexander et al.: Cultural Trauma And Collective Identity. Mary Ballou and Laura S. Brown: Rethinking Mental Health \& Disorders: Feminist Perspectives. Gert Buelens et al.: The Future of Trauma Theory: Contemporary Literary and Cultural Criticism. Stef Craps: Postcolonial Witnessing: Trauma Out of Bounds. Richard Crownshaw et al.: The Future of Memory. Roger Luckhurst: The Trauma Question. Michael Rothberg: "Decolonizing Trauma Studies: A Response." Rebecca Saunders: Lamentation and Modernity in Literature, Philosophy, and Culture.
} 
emerging from studying the Holocaust, the field has largely overlooked traumatic events occurring outside of Europe or the United States (e.g., the Syrian Civil War, the Congolese Civil War, the Rwandan Genocide), or non-majority experiences of traumatic events occurring in the Western hemisphere (e.g., Navajo experiences of settler colonialism in the southwestern United States). Thus, working to fulfill the field's "promise of cross-cultural engagement" is a project of recognition and remembering, of bringing into consciousness traumas outside of Euro-American settings and/or marginalized experiences of Euro-American traumas. In addition, fulfilling the field's promise is a project of allowing that recognition and remembering to transform assumptions about, sites of analysis for, and ways of engaging with trauma.

While trauma theory increasingly engages with literature, testimonies, and histories beyond the Nazi Holocaust as well, such as the September 11, 2001 destruction of the World Trade Center in New York City, it continues to focus on white and/or male experiences of such events through theories built in studying the Nazi Holocaust, applying those theories to other catastrophes, and assuming the universality of traumatic experience. Moreover, a singular focus on anti-narrative and an avoidance of realism, evident in the prevailing modernist aesthetic in the field, obscures "specific social and historical contexts in which trauma narratives are produced and received," and fails to account for "the diverse strategies of representation and resistance which these contexts invite or necessitate" (Craps 43). As Euro-Americanism erases crucial differences among forms of and responses to trauma, the field risks "assisting in the perpetuation of the very beliefs, practices, and structures that maintain existing injustices and inequalities" (ibid). Euro-Americanism produces a kind of theoretical colonization, reinforced by the 
naturalized universalization of Western (bio)medical conceptions of trauma as individual pathology — an orientation that leads to trauma theories and treatments that are inappropriate, lead to false conclusions, and obscure suffering.

This dissertation illustrates the harm of universalizing Western (bio)medical conceptions of trauma, extending interrogations of Euro-Americanism in the field of trauma theory by way of spotlighting female experiences of sexualized trauma in different global settings. Spotlighting textual representations of sexualized trauma can dislodge the hold of Euro-Americanism in trauma theory, because sexualized violence disproportionately impacts historically underrepresented groups in the field, including girls, women, people who identify as LGBTQ, and gender nonconforming people. Moreover, spotlighting textual representations of sexualized violence illustrates how trauma emerges within a matrix of oppressions based on intersections of race, ethnicity, gender, class, sexuality, age, geographic location, and other naturalized identity markers "work[ing] together in producing injustice," as Patricia Hill Collins notes in Black Feminist Thought: Knowledge, Consciousness, and the Politics of Empowerment (18). Finally, this dissertation engages textual representations of sexualized violence as a means of challenging trauma theory's Euro-Americanism because the textual representations it examines chafe against the field's tendency to configure fragmentation and aporia as primary representational devices in traumatic literature and film.

Following Donna Haraway's, Jasbir K. Puar's, and other assemblage theorists' critiques of feminism's reliance on a notion of common female experience, this dissertation importantly extends trauma theory beyond the singular survivor-beyond individual sexually violated bodies. Paraphrasing Haraway, Puar explains that the human 
body is not a "discrete organic thing"- that "the body does not end at the skin"-which evidences the need for ecology, rather than individualism, as a paradigm for understanding the trauma of rape (57). That is, sexualized trauma emerges within and affects whole systems of bodies that include and exceed the human. An assemblage approach to sexualized trauma, which this dissertation enacts, shifts attention to patterns and relations - sets of conditions (i.e., discursive formations) - that make sexualized violence possible. As Puar writes, "the focus [should be] not on whether there is a crime taking place, nor determining who is at fault, but rather asking, what are the affective conditions necessary for [rape and other forms of sexualized violence] to unfold?" (61). This dissertation aims to dislodge sexualized trauma from its naturalized "event-ness," its particular subjects, and beyond other static biomedical conceptions of trauma, such as those found in DSM-5.

Naturalized medical trauma discourses overlook the conditions that enable sexualized violence, among other forms of violent oppression, focusing instead on isolated instances or enactments of violence, as well as on progress narratives of individual survivors moving on in the aftermath of catastrophe. Representations of sexualized violence in mainstream media, including the mainstream antiviolence movement, tend to isolate and contain victims in their victimization. The individualization of trauma perpetuates victimization.

If the ultimate goal for trauma survivors, and for the field of trauma theory more broadly, is to shift from "victim mentality" or what Shoshana Ringel and Jerrold R. Brandell refer to as " "victim mythology" " in Trauma: Contemporary Directions in Theory, Practice, and Research, toward a more nuanced understanding of the diverse 
strategies through which we experience and represent violence, then we need more flexible conceptions of trauma and healing. We need to revise naturalized narratives of chronic fear of negativity, harm, or danger from the external, which can keep people imprisoned in the past. Collectivizing traumatic experience, a revision strategy this dissertation proposes, positions empowered vulnerability - a conscious, willing openness to injury, change, growth — as a viable alternative to victim mythology. Collectivizing traumatic experience uproots trauma from its individualistic confines-victims and perpetrators, as well as advocates, law enforcement agents, and medical professionals who interface with trauma victims and perpetrators - calling attention to the social conditions that make sexualized violence possible in the first place, and making space for forms of healing that transcend (or bypass altogether) the isolation of the doctor/patient relationship that is so central to modern medicine.

As a healing strategy, collectivism provides a corrective to the universalization of Western psychoanalytic conceptions of trauma and recovery, and to the dearth of analyses that highlight collectivist approaches to healing trauma, in conjunction with or even in lieu of interpersonal therapies, medical and legal interventions (e.g., rape kits, restraining orders), and assistance from social programs (e.g., battered women's shelters). Collectivism emphasizes interdependence, a counterpoint to individualism pervading Western mental healthcare in what feminist psychologists Mary Ballou and Laura S. Brown call its "corporate model of illness," the treatment of health as commodity (xviii). In Toward Psychologies of Liberation, psychologists Mary Watkins and Helene Shulman describe how this corporate model prescribes individualized trauma treatments that focus on curing a singular subject (patient/survivor) by way of bringing a singular narrative 
(event) into consciousness: “The therapeutic fantasy shared by many psychologists appears to be that there is a lost narrative of the traumatic past locked like an abscess within the psyche. Dissociated, but still accessible to the narrative ego, attempts to remember the past are the basis for much work of individual counseling" (108-09). This psychotherapeutic approach of personal remembering and telling relies upon Freudian concepts of dissociation and the talking cure underlying Western theories of trauma. Thus, challenging the alleged universal efficacy of psychotherapy can disrupt EuroAmericanism in trauma theory by exposing the limits of and speculating about alternatives to dissociative conceptions of trauma.

In contrast to psychotherapy, collectivist approaches to trauma and recovery assume that healing begins with entire communities that witness or survive catastrophic events. Collectivist approaches can include public memorials, demonstrations or protests, consciousness-raising groups, or other community activities that involve collaboration and raise awareness. Collectivist ways of healing remind us that the oppression of sexualized trauma, for example, affects people in social settings, and cannot be resolved or prevented (solely) through (bio)medicine. As Watkins and Shulman argue, traumas of contemporary global life, such as war and mass displacement, demand collectivity. They describe the limitations of conventional psychotherapy for healing trauma, explaining that,

Psychological scars and post-traumatic stress from war, violence, terror, genocide, sudden toxic pollution, natural disaster, and resultant displacement and forced migration have led to a need for psychological practices that can repair the bonds among people as well as the narrative threads of an individual life history. Lost 
rituals, social networks, beliefs, and trust are not only individual but collective issues, and cannot be rebuilt in private spaces alone. (14)

While accounting for non-individualistic, non-Western effects of traumatic events, such as lost rituals and fractured social networks, Watkins and Shulman remain prescriptive in their theory that private spaces for healing are insufficient. They are Western-trained psychologists invoking naturalized individual/collective binaries, prescribing an "add on" to current models (e.g., in their call for "psychological practices that can repair the bonds among people as well as the narrative threads of an individual life history"), rather than transforming current models altogether. Moreover, their liberation psychology framework still imposes an externally-developed, preexisting model onto lived experience, inhibiting and even altogether prohibiting lived experience from transforming the model altogether. In light of the pervasive Western medical model that prescribes individual treatments for trauma-related disorders, such as those Watkins and Shulman identify, collectivism becomes a particularly vital concept for healing sexualized trauma. As this dissertation seeks to show, we need collectivism so that we can do more; we need to move beyond individual/collective, private/public binaries altogether as trauma cannot be understood outside of its embeddedness in social conditions.

Collectivism is an ethical response to violence. In Precarious Life: The Powers of Mourning and Violence, Judith Butler argues that violence should prompt recognition of our "inevitable interdependency" (xii). In the wake of the September 11, 2001 attacks on the World Trade Center, Butler explains that the U.S. "miss[ed] an opportunity to redefine itself as part of a global community," turning instead to increased nationalism, surveillance, censorship, and other forms of state control (xi). Butler goes on to argue for 
a nonviolent ethics "as the basis for global community," which can be embraced through critical discourse, public debate, and reflection on "what counts as a livable life and a grievable death" (xv). Butler's argument helps establish collectivism as an ethical means of engagement in an increasingly globalized world. Collectivism diffuses the graspingfor-control that perpetuates the foreclosure of critique, which enables violence in the first place. In World Memory: Personal Trajectories in Global Time, Jill Bennett and Rosanne Kennedy claim "memory is a product of the intersection between the global and the local, the collective and the lived bodily experience" (15). Their argument extends Butler's advocacy of interdependence in the face of violence-in this case, the interdependence of global and local, collective and personal— to argue for a collective memory of trauma that includes but does not end (or begin) with the so-called "individual." Even in death, the resistance of connection and community remain as the dead die fighting, refusing to be separated. "Our inevitable interdependency" is resistance.

Challenging the (bio)medicalization of trauma, specifically the individualization and pathologization of suffering, requires a conscious openness not only to encountering injury and death—especially traumatic events and embodied traumatic experiences marginalized in trauma theory and practice — but also to enormous variation and multiplicity across the ways in which humans experience, remember, mourn, transmit, and otherwise make meaning of suffering. To cultivate such empowered vulnerability, the field of trauma theory needs to engage in more investigative and descriptive methodologies, as distinct from prescriptive and evaluative ones. This dissertation enacts such a methodological shift, investigating and describing differing discursive formations of trauma in medical documents, literary texts, and films, and assessing naturalized, 
normalized trauma wisdom across different texts and their contexts. To that end, the following chapters examine textual representations of trauma as communications that produce particular cultural understandings of trauma, and that expose the relationship among embodied experiences, theories, and Western (bio)medical conceptions of trauma as at once coinciding and dissonant. Turning to medical, literary, and cinematic texts on trauma to understand girls' and women's diverse experiences of sexualized violence works to undo dominant paradigms for representing and responding to trauma, such as the widespread assumption that criminalizing sexualized violence will minimize or eradicate it altogether.

Chapter two exposes universalized Euro-American, modernist ideologies that configure trauma as a personal failure to assimilate catastrophic events, in the American Psychological Association's clinical definition of posttraumatic stress disorder (PTSD) outlined in The Diagnostic and Statistical Manual of Mental Disorders (DSM-5) and its supplementary diagnostic guide, The Pocket Guide to the DSM-5 Diagnostic Exam. The defining discourse of medical knowledge about trauma, DSM-5 functions as a standardizing authority on mental health globally. Its influence on contemporary trauma theory and practice cannot be underestimated.

Chapter three problematizes the pathologization of trauma survivors naturalized by DSM-5, illustrating how Stieg Larsson's Girl with the Dragon Tattoo and Niels Arden Oplev's and David Fincher's film adaptations condition us to perceive trauma as a systemic pathology that catalyzes feminist vigilantism. These texts expose the ineffectiveness (and outright exploitation) of institutions like the healthcare industry that allegedly serve the public good. Girl with the Dragon Tattoo is notable within 
mainstream novels and films representing rape, given its extensive depiction of feminist vigilantism as a viable response to sexualized violence. A case study of Dragon Tattoo is important in challenging the naturalized pathologization of rape survivors, and in extending understandings of how survivors reject victimization by attempting to transform the very systemic dysfunctions responsible for bringing about the trauma.

Chapter four demonstrates how collective acts of memorialization transform systemic pathologies that produce sexualized violence, showcasing co-created trauma testimonies in Nava Semel's hybrid Nazi Holocaust novel, And the Rat Laughed. Where Dragon Tattoo represents trauma as an institutional (medicolegal) failure, Rat collectivizes traumatic experience itself, representing how institutionalized anti-Semitic misogyny enables rape, but also how the trauma brought about by rape surpasses individuals who are raped and their particular stories of sexualized victimization. Any contemporary study of trauma would be remiss without the inclusion of Nazi Holocaust literature, and And the Rat Laughed is particularly conducive to an analysis of sexualized Nazi Holocaust trauma given its primary theme of child sexual abuse. Moreover, Rat's obscurity within the genre - relative to texts like Anne Frank: The Diary of A Young Girl, or Cynthia Ozick’s widely anthologized “The Shawl," among other canonical works about female Nazi Holocaust experiences_-underscores the importance of case studying such a text in a dissertation attempting to complicate naturalized trauma wisdom.

This dissertation concludes with a fifth chapter that articulates rhetorical strategies for teaching traumatic texts, demonstrating how Alan Duff's Once Were Warriors and Lee Tamahori's film adaptation call upon students to bear witness to internalized oppression rampant in indigenous groups colonized by Europeans. In contrast to Nazi 
Holocaust trauma, colonial trauma is often underprivileged in the field of trauma theory. Once Were Warriors is a particularly salient text to examine in challenging EuroAmericanism in the field, insofar as the novel and film represent sexualized trauma along intersections of the interpersonal and the structural. Protagonist Beth Heke appears to embody the misogynist stereotype that women want to be abused, and that women "get off on" being raped. Yet Once Were Warriors narrates Beth's attachment to her violently abusive husband, Jake Heke, with themes of colonial displacement, suggesting an irreducible matrix of oppressions based on Beth's love for Jake, her Mäori identity, and the realities of poverty and violence afflicting many Mäori groups in contemporary New Zealand. In this final chapter in particular, this dissertation contributes to trauma pedagogies, particularly the teaching of textual representations of sexualized violence in U.S. university settings where women are at high risk of being sexually assaulted, ${ }^{3}$ and proposes pedagogical strategies for teaching students to critically read and write about trauma as political acts of bearing traumatic witness.

Ultimately, this dissertation is a project of reconciliation, of rendering inextricably linked the bifurcation of the familiar and unpredictable, the local and global, the personal and cultural, the private and political. Isolating trauma from its historical, cultural specificities, and universalizing (bio)medical understandings of trauma pathologizes survivors, silences histories, obscures the infinite range of possible responses to catastrophe, confines understandings of what "wound" means, and otherwise causes harm. This dissertation proposes collectivizing trauma as a corrective, a means of bridging differing discursive formations of trauma in DSM-5, Girl with the Dragon

\footnotetext{
${ }^{3}$ According to a Time Magazine article, "The Sexual Assault Crisis on American Campuses," 1 in 5 college women is the victim of attempted or completed sexual assault (1).
} 
Tattoo, And the Rat Laughed, and Once Were Warriors. Collectivizing trauma centralizes the social conditions that bring about catastrophe, rather than its survivors, and makes space for forms of healing and resistance that affirm our fundamental interdependency. 


\section{CHAPTER II}

\section{A CASE STUDY OF MEDICAL TRAUMA DISCOURSES: DISCURSIVE} FORMATIONS OF TRAUMA IN THE DIAGNOSTIC AND

\section{STATISTICAL MANUAL OF MENTAL DISORDERS V}

Mental healthcare is rapidly growing as a branch of medicine, in the United States and globally. Every year, the U.S. alone spends approximately $\$ 113$ billion on mental health treatment, most of which goes toward prescription medications and outpatient treatment (Kliff). Despite this significant monetary investment, for patients, mental healthcare costs can prove financially and psychologically prohibitive. In contrast to other physiological forms of medical care, care for mental illness is inconsistently covered by insurance providers and, coupled with a profound stigma attached to mental illness, deters many people from even seeking treatment in the first place. ${ }^{4}$ Many opt to cope with psychological distress on their own. Reinforcing the notion that mental healthcare is non-essential, states often cut mental healthcare budgets during times of economic crisis. Mental healthcare is costly and stigmatized, yet also "not big enough to serve all those who need it" (ibid). We might say that mental healthcare costs more than it cares. ${ }^{5}$

\footnotetext{
${ }^{4} 66 \%$ of patients who considered seeking treatment chose not to because they believed "the problem would get better on its own" (Kliff).

${ }_{5}^{5}$ For a more in-depth discussion of the state of American healthcare, see Ballou \& Brown; Barr; Bureau of Labor Statistics; Heiffron \& Brown; Garfield; Greene; Ibrahim \& Morrow; Interlandi; Klaver; Mark et al.; Porter; Potter; Sareen et al.; Segal; Teich; van der Kolk; Watkins \& Shulman; and World Health Organization.
} 


\section{DSM: An Epistemo-Politics of Health and Wellness}

By medicalizing mental health, what Clarke et al. describe in "Biomedicalization: Technoscientific Transformations of Health, Illness, and U.S. Biomedicine" as "processes through which aspects of life previously outside the jurisdiction of medicine [e.g., mental illness] come to be construed as medical problems," the Diagnostic and Statistical Manual of Mental Disorders (DSM) represents, in part, an ongoing effort to close the gap between need and available resources (47). Widely regarded as psychiatry's bible, DSM provides “descriptions, symptoms, and other criteria for diagnosing mental disorders" (“American Psychiatric Association”). DSM evolved as a taxonomy of socalled mental disorders; and its first edition, published in 1952, coincided with the increasing involvement of psychiatrists in treating World War II soldiers experiencing Combat Stress Reaction (CSR), otherwise known as battle fatigue (Friedman). Over the sixty-four years that $D S M$ has been in print, the manual has paved the way for psychological distress to be recognized as a medical condition, which has prompted insurance providers to increasingly cover treatment, and patients to increasingly seek out treatment.

That said, ideology about what mental illness is, its symptomatology, what it means to have and who has the power to diagnose and treat mental disorders permeate $D S M$. The twenty-some categories or disorder groups that DSM organizes itself aroundfrom Depressive Disorders and Anxiety Disorders, to Sexual Dysfunctions and Disruptive, Impulse-Control, and Conduct Disorders - are socially constructed around imagined norms about health and wellness. Moreover, such categorization replies upon the power to diagnose. DSM, thus, is a politics of mental health with epistemic 
dimensions - what I refer to as an epistemo-politics of mental health — insofar as the medical knowledge $D S M$ represents is both generated and validated through racialized, gendered, classed relations of power. DSM is written by and for healthcare professionals, primarily for use in clinical settings, positioning the manual as the arbiter of mental healthcare standards worldwide. Its producer, the American Psychiatric Association (APA), explains:

[T]he handbook is used by healthcare professionals in the United States and much of the world as the authoritative guide to the diagnosis of mental disorders. DSM contains descriptions, symptoms, and other criteria for diagnosing mental disorders. It provides a common language for clinicians to communicate about their patients and establishes consistent and reliable diagnoses that can be used in the research of mental disorders. It also provides a common language for researchers to study the criteria for potential future revisions and to aid in the development of medications and other interventions. (emphasis added "American Psychiatric Association")

The APA's language here, in its statement of purpose for the manual, reveals several interlocking assumptions about mental healthcare, as well as the role $D S M$ plays in both constructing and reinforcing those assumptions. While people and texts reciprocally shape realities, and DSM language alone does not automatically or intrinsically enforce assumptions about mental health—language can only do this work if people and tools act and behave together in ways that shape language into reality-the cultural, medical 
capital DSM garners suggests that an analysis of its discourse can reveal much about how we think about the psyche.

Firstly, the APA's statement decontextualizes mental illness, pointing up an underlying assumption that people experience psychological distress in the same way everywhere. DSM's status as "the authoritative guide" and its provision of "a common language" for clinicians and researchers override sociocultural variations around what mental disorders are- if they even exist as such in a given cultural setting. Moreover, the APA's statement homogenizes understandings of mental illness across the globe, including but not limited to the notion that mental illness is a primarily psychological and not physiological or even sociocultural phenomena, valorizing the West as the most advanced region of the world when it comes to mental healthcare. The structure of the very first sentence, "the handbook is used by healthcare professionals in the United States and much of the world," positions the West as the global leader of healthcare standards, which then extend to other countries. As a case in point, the last sentence of the APA's statement of purpose - that DSM works "to aid in the development of medications and other interventions"- establishes pharmaceuticals as the preferred intervention, thereby lumping the vast range of non-medical, non-Western interventions (such as alternative therapies like acupuncture or meditation) into the single, non-distinct category of "other." In this way, DSM reifies the colonial ideology that the West is more civilized, more skilled, more educated, and in possession of better resources than the rest of the world. In emerging in Western global settings, the institution of psychiatry "works in the interest of privilege and power to cross cultural, political, and textual borders so as to deny the specificity of the other and to reimpose the discourse and practice of colonial hegemony" 
(Giroux 15-16). That is, the European colonization of indigenous lands and cultures coincides with the medicalization of mental health, as numerous studies suggest. ${ }^{6}$ Features of a colonizing taxonomy include the mind/body split (i.e., Cartesian Dualism), the universalization of bodies and the pathologization of individuals, and the classification of "other" (i.e., disease, disorder, dysfunction) as stranger, as subhuman.

In addition to decontextualizing and homogenizing mental illness, the APA's statement about the purpose of DSM also universalizes a language for mental healthcare, the language of $D S M$, which presumes that universalizing this language will produce more accurate diagnoses and treatments of mental illness. Yet many healthcare professionals contest $D S M$, finding that it creates more problems than it resolves. Chair of the DSM-IV Task Force and former Chair of Psychiatry at Duke University, Allen Frances, M.D. asserts that the manual produces "massive over-diagnosis," including what he calls "fad diagnoses," and "harmful over-medication." He goes as far as to claim that "DSM-5 violates the most sacred (and most frequently ignored) tenet in medicine-First Do No Harm!" DSM may be making people more sick than well.

As the APA acknowledges in the manual's introduction, "[a]lthough some mental disorders may have well-defined boundaries around symptom clusters, scientific evidence now places many, if not most, disorders on a spectrum" depending on a patient's familial and sociocultural norms (DSM-5 6). In other words, patients' backgrounds affect the degree to which they are "ill." The APA goes onto explain that "boundaries between normality and pathology vary across cultures"-for example, tolerance for particular symptoms or behaviors - suggesting that what constitutes dysfunction in one cultural

\footnotetext{
${ }^{6}$ For more on the colonization of psychiatry, see Ibrahim \& Morrow; Porter; Potter; and Sherwood.
} 
setting may not in another. The APA's construction of mental illness as a spectrum implies a contradiction of its stated purpose, "establish[ing] consistent and reliable diagnoses," which further implies that mental healthcare is at once culturally variable and a precise science. We might thereby understand $D S M$ as a colonizing imperial force - a non-human agent or actor within the institution of psychiatry/psychology in particular, and of Western biomedicine in general. However, the latter connection is fraught because medical diagnoses seek to be accurate explanations of pathologies that can be identified across a wide spectrum of patients. When an understanding of pathology as precise and diagnosable is combined with psychosocial disorders, and when DSM bifurcates these disorders from more purely physiological ones, the validity of $D S M$ 's stated purpose to establish consistent, reliable diagnoses becomes suspect. DSM is not so much a precise science, as it is an epistemo-politics of purity — a manifestation of a belief that clear boundaries can be identified for different kinds of illnesses and their treatments, across sociocultural settings.

Similar to the APA's statement of purpose, the APA's very definition of mental disorder also reveals several interlocking assumptions_-about mental health itself, as well as the role DSM plays in both constructing and reinforcing those assumptions. In order for clinicians to diagnose someone with a $D S M$ disorder, the patient must meet diagnostic criteria listed for that specific disorder, in addition to all criteria in the manual's general definition of mental disorder:

A syndrome characterized by clinically significant disturbance in an individual's cognition, emotion regulation, or behavior that reflects a dysfunction in the psychological, biological, or developmental processes 
underlying mental functioning. Mental disorders are usually associated with significant distress or disability in social, occupational, or other important activities. An expectable or culturally approved response to a common stressor or loss, such as the death of a loved one, is not a mental disorder. Socially deviant behavior (e.g., political, religious, or sexual) and conflicts that are primarily between the individual and society are not mental disorders unless the deviance or conflict results from a dysfunction in the individual, as described above. (emphasis added, DSM-5 20) From this definition, it follows that developing a mental disorder stems from a mental, rather than a sociocultural or other environmental, dysfunction. Released in May 2013, the current edition of $D S M, D S M-5$, includes diagnostic criteria for more than twenty different types of mental disorders, each of which includes multiple sub-classifications, for a total of several hundred possible mental disorder diagnoses. ${ }^{7}$ The plethora of diagnoses, coupled with the APA's stipulation that patients meet a range of specific and general diagnostic criteria, may easily produce inconsistent, inaccurate diagnoses - the very opposite outcomes the APA claims to establish.

We can detect a potential for diagnostic inaccuracy in how DSM actively constructs more than it describes the very profession of mental healthcare. The APA bifurcates psychological and physiological suffering, implying that the two are fundamentally separate. The very term mental disorder reduces cognitive, emotional, and behavioral disturbances to mental ones, overriding the interdependence of mind and body. In so doing, the manual fallaciously isolates the brain from organs, muscles, bones,

\footnotetext{
${ }^{7}$ For example, the $D S M-5$ section on Anxiety Disorders includes twelve different diagnoses.
} 
tissues, and other parts of the body that can both cause and affect psychological distress. In the case of posttraumatic stress disorder (PTSD), the primary disorder analyzed in this chapter, such a split is especially problematic as traumatic memories are stored in the body. Globally-renowned traumatologist, Bessel A. van der Kolk, M.D., studies the traumatized brain through neuroimaging. His research suggests that traumatic experience alters the brain's threat-perception system and thus, fundamentally reshapes how the body functions (67). To quote the title of his groundbreaking publication on healing trauma, "The Body Keeps the Score." DSM reinforces a Cartesian dualism, which can lead to diagnoses that fail to identify how mind and body work together in manifesting so-called traumatic disorders.

Moreover, the APA individualizes mental health, locating the cause of mental disorders in individual dysfunctions - in "psychological, biological, or developmental processes" rather than in sociocultural, institutional, or other environmental ones. For survivors of sexualized violence who are diagnosed with $\mathrm{PTSD},{ }^{8}$ such an assumption about the origins of mental illness pathologizes the person already suffering, locating dysfunction within the survivor rather than in the systemic misogyny that produces and perpetuates rape. In this way, $D S M$ reinforces yet another false binary, between the individual and society, implying that the two function independently of one another. While it would be impossible to determine precisely where are how individual and society intersect in daily functioning, the individualization of dysfunction can lead to diagnoses that fail to identify how social norms contribute to and manifest certain disorders.

\footnotetext{
${ }^{8}$ In working with rape survivors, many care providers may refer to Rape Trauma Syndrome (RTS) or Complex Posttraumatic Stress Disorder (C-PTSD) instead of PTSD, although neither of these more rapespecific diagnoses is included in DSM-5.
} 
$D S M$ also pathologizes the individual. The APA's caveat that "socially deviant behavior and conflicts that are primarily between the individual and society" do not count as mental disorders reinscribes commonplace binaries that can interfere with clinicians accurately assessing their patients' symptoms. This caveat may represent the APA's attempt to underscore how DSM cannot and should not determine which behaviors are deviant in any given social setting and then pathologize those behaviors - as it did, for example, by classifying homosexuality as a mental disorder in DSM-I and DSM-II. However, this caveat also raises questions around whether developing a taxonomy of socalled "mental disorders" is even appropriate in the first place. The APA positions itself in an impossible space, in which it must keep to narrow, internalized definitions of mental illness in order to avoid social stigmatization of certain non-pathological behaviors (e.g., homosexuality), at the same time as it must take into account varying understandings of so-called "deviance" across diverse familial, cultural, and political settings. Separating "deviant behavior" from mental illness, the individual from society, the manual suggests that clinicians should avoid linking the two. One could interpret the APA's caveat as follows: A socially deviant person is not mentally ill, and a mentally ill person is not socially deviant. For a rape survivor who engages in socially-deviant behaviors in response to the trauma of rape-for example, protagonist Lisbeth Salander in Girl with the Dragon Tattoo, who responds to rape with rape-this caveat may pathologize her attempts to defend herself and seek justice for other (potential) victims of misogynist violence. As I discuss in the next chapter, women like Lisbeth who endure rape within a culture that systematically enables it (Lisbeth's primary perpetrators are her legal guardian and her psychiatrist), DSM's definition of mental disorder may re-inflict 
violence upon her; it may prematurely eliminate a possible PTSD diagnosis that could help her receive treatment, and/or the possibility that her behavior is a viable, nondysfunctional response to systemic injustices.

Yet just as $D S M$ individualizes mental health and pathologizes the individual with mental illness, it ultimately defers to sociocultural norms to determine the degree to which a person is labeled "ill." The manual stipulates that these norms, more than its own classification of mental disorders, shape whether an individual's response to a stressor, "such as the death of a loved one," is "expectable" or "approved" and therefore not a mental disorder (DSM-5 20). DSM even assesses suffering through a sociocultural lens, looking to "social, occupational, or other important activities" to determine the extent or severity of dysfunction (ibid). Nonetheless, the manual fails to delineate biological, chemical and genetic factors from environmental, socially constructed ones in how it describes different disorders. In these ways, DSM invites dissent, especially the conviction that many clinicians hold: that mental healthcare is not a precise science but rather, an ideology — a materialization of our Western fixation on self. Thus we might say that the APA pays lip service to sociocultural variation, but in practice, lets itself off the hook of actually establishing consistent and reliable diagnoses that can be used across different sociocultural settings.

The APA's statement of purpose, as well as its definition of mental disorder, exposes DSM as a discursive construction. In short, DSM is an epistemo-politics of mental, emotional, behavioral health. Medical professionals themselves corroborate this perception. As medical student Zoë Cruse notes in her blog, "The Forsaken Specialty: Why Do Doctors Look Down on Psychiatry?," psychiatry is not often regarded as "a true 
profession—-as] the consultants sit and sip tea, talk nonsense, and nobody ever seems to gets better. No blood test confirms what is wrong. No imaging shows the diagnosis.' " The difficulty of proving that a mental illness exists, not to mention the increasing suspicion around the alleged proven efficacy of talk therapy for treating conditions such as posttraumatic stress disorder (PTSD) and depressive disorders, ${ }^{9}$ render mental healthcare highly unstable. Clinicians rely on behavioral symptoms and patient testimonies to diagnose mental disorders, and to determine and assess treatments. The precision of bloods tests, X-rays, MRI's, ultrasounds, and other diagnostic tools used in physiological branches of medicine cannot reliably show mental illness. DSM represents an effort to establish psychiatry as tantamount to other branches of medicine. This effort, however, may further marginalize mental healthcare from other medical branches, and prevent patients from receiving effective care.

To explicate how trauma is socially constructed as a mental disorder, I conduct in this chapter a Foucauldian analysis of DSM-5, analyzing how this latest edition of the manual constructs posttraumatic stress disorder (PTSD). Drawing on Michel Foucault's genealogy of modern medicine, The Birth of the Clinic, and his archaeology of discourse, The Archaeology of Knowledge, the next section of this chapter explicates how medical knowledge is more socially constructed than biologically determined. Section three explicates how $D S M-5$ bifurcates normal and pathological functioning. As Foucault explains, modern medicine functions as a model of normalcy and not of health, bifurcating "normal" and "pathological" bodies. Finally, section four analyzes how DSM5 defines and recommends diagnosing PTSD, bifurcating normal and pathological

\footnotetext{
${ }^{9}$ For more on the inefficacy of talk therapy, see Benedict Carey's New York Times article, "Effectiveness of Talk Therapy is Overstated, a Study Says."
} 
responses to traumatic events. In this section, the actual case study of $D S M-5$, I follow several principles of Foucauldian discourse analysis. I "describe the specific authorities that guided [the APA's] choice[s]" and that regulate how DSM-5 is produced, circulated, and received (Archaeology 66). I also describe, "what the discourse must carry out in a field of non-discursive practices"- the rules and processes of appropriation surrounding the manual, which have material consequences on people's lives (Archaeology 68). I then "determine possible points of diffraction of discourse," or where the discourse confronts or avoids contradictions, obstacles, and even openings that may enhance our understanding of mental illness (Archaeology 65). To paraphrase Foucault, to analyze $D S M-5$ 's construction of trauma is to analyze the professional and public roles the manual plays, how the manual is taken up, and how the manual classifies PTSD in particular.

\section{The Social Construction of Medical Knowledge}

In the eighteenth century, Western medicine shifted from treating the individual as incidental to disease to treating the individual as the source of disease. The evolution of medical knowledge about trauma generally and PTSD in DSM in particular exemplifies this shift. While trauma has long-been part of the human condition, psychiatric theory and practice did not formally recognize psychological trauma as a medical condition until the late 1800's (roughly 1860 through 1893) when French physician Jean Martin Charcot, "the most famous European neurologist of the era," examined the relationship between trauma and mental illness in female patients diagnosed with hysteria (Luckhurst 34). Before Charcot, doctors treated hysteria patients 
with hysterectomies, removing the organ thought to be diseased: the uterus. Charcot's research challenged the efficacy of such interventions, exposing hysteria symptoms as psychological rather than biological in nature. The focal point became the hysteria patient herself, who was conflated with her brain, not her allegedly diseased uterus. She became pathological. Medicine enacted a misconception of the source of disease-from treating the uterus as diseased organ, to treating the person impaired by the malfunctions of a diseased organ.

Trauma was not formally recognized as a medical condition - a mental disorderuntil 1980, when the APA added PTSD to DSM-III, classifying it as an anxiety disorder. This addition facilitated recognition of survivors' traumatic experiences of the Nazi Holocaust, Vietnam War, natural- and human-made disasters, in addition to other experiences DSM-III labeled "outside the range of usual human experience" that "would evoke significant symptoms of distress in most people," as attributable to an outside event rather than to an inherent individual neurosis (DSM-III 236). Of course, including PTSD in a manual of mental disorders suggests that trauma is, to refer back to the DSM definition of mental disorder, about individual dysfunction (cognitive, emotional, behavioral). Yet the addition of PTSD made it possible for clinicians to diagnose and treat survivors for psychological trauma, facilitating coverage of care from insurance providers. The DSM-III inclusion of PTSD as a medical, psychological condition (an anxiety disorder) illustrates how conceptions of mental illness change over time in relation to how we understand mind, body, and human experience.

In particular, the addition of PTSD to DSM-III emerged from a classificatory medical model. Such a model transforms medical knowledge, discourse, and practices 
into discrete diseases and disorders, which are in turn transformed into codes used for ease of communication among medical professionals, including insurance billing purposes. ${ }^{10}$ This model is based upon a very particular ideology around life and death, health and disease: the anatomo-clinical method. "[A] two-part methodology that link[s] clinical signs," symptoms documented and observed over time, "with anatomical lesions" discovered postmortem through an autopsy of the brain and spinal cord, the anatomoclinical method relies ultimately upon disease, upon death, to understand health (Goetz 203). Dualism underlies the anatomo-clinical method.

Death enables a clear vision of life, as Foucault explains in Birth of the Clinic. Disease, examined through the autopsy, now has "a mappable territory, a subterranean, but secure place where its kinships and consequences are formed" (Birth 149). To see death is to know life. Thus, we can understand disease as "life undergoing modification" (Birth 153). A complete reversal of prior wisdom, medical knowledge now suggests that people become ill because they die; they do not die because they become ill. A rape survivor, for example, may develop PTSD because she will die. She does not die because she develops PTSD. Death, then, is the root of trauma. DSM-5 reinscribes this understanding of disease, defining trauma through mortality, as "actual or threatened death, serious injury, or sexual violence" (Birth 271). The emergence of a classificatory medical model changes how we understand disease. Disease is no longer something to try to eliminate or extract, but to understand and describe. Put simply, disease is now recognized as a byproduct of being human — an inevitability.

${ }^{10}$ For example, DSM-5 uses code 309.81 for PTSD. 
The classificatory medical model operates on the basis of what Foucault calls the medical gaze or the gaze. The gaze_- "taken as a simple, unconceptualized confrontation of a gaze and a face, or a glance and a silent body; a sort of contract prior to all discourse"-generates knowledge about the body by way of the clinician's observations of it (Birth xv). The gaze penetrates, seeing through the surface reality to an underlying truth, endowing clinicians with immense power to look. Patients, in turn, implicitly grant doctors visual access to their bodies, without question, as we have been conditioned to accept the gaze as intrinsic to medicine. Looking and knowing are the doctor's purview, not the patient's. The gaze travels in one direction, from doctor to patient, positioning the individual at the center of medical knowledge, while simultaneously reducing the individual to her $\leftrightarrow$ his ailment. The body becomes "the world of objects to be known" as the gaze renders disease a geography, a landscape to be mapped through looking (Birth $\mathrm{x})$. To see is to know, to know is to enact power, as "the gaze that sees is a gaze that dominates" (Birth 49). The medical gaze renders patients passive objects of spectacle, a platform for doctors to advance medical knowledge, to advance their own field, and their careers. The gaze constructs a nonreciprocal relationship between doctor and patient, and functions to serve the clinician's more than the patient's interests. In short, the medical gaze is premised on the notion that the world is something to be classified, controlled, and consumed. DSM classifications of mental disorders enact such a gaze.

The medical gaze can be characterized, then, by a perceptual shift from diseases to bodies, setting in motion "the endless task of understanding the individual" (Birth 15). This "endless task" conflates patient and illness, disease and pathology, person and diseased organ (the brain). Mental healthcare practitioners study, for example, $a$ 
traumatized patient rather than trauma as a disease. Yet people are more likely to treat illnesses if they are seen as manageable aspects of life - as things they have, PTSD, rather than things they are, traumatized. The rhetoric of battle, fighter, recovery, and survivor - common terms in trauma discourse - provides but one example of such a cultural identification with illness. If a patient does not survive a disease such as cancer, this rhetoric can translate as a personal failure to fight off disease. If a patient does survive, this rhetoric marks the body permanently through disease, whether or not cancer still exists in the body. The gaze keeps us chronically sick. "The endless task of understanding the individual" makes disease profitable. If disease is an inevitable byproduct of being human, then it follows that medical professionals have much to gain from keeping people sick.

The diagnostic process for mental disorders illustrates this potential for profiting from keeping people sick. Abraham M. Nussbaum, M.D., author of the $D S M-5$ companion text, The Pocket Guide to the DSM-5 Diagnostic Interview, explains that psychiatrists and other mental healthcare practitioners use the diagnostic interview to make diagnoses of mental illness. During this 30-minute session, the medical practitioner asks the patient a series of questions to assess the presence and degree of "signs that indicate the dysfunction of neural circuits" (Nussbaum 5). Upon making a diagnosis, the clinician develops a treatment plan, which often includes forms of ongoing care (e.g, psychotropic medications, regular therapy sessions). The interview facilitates diagnosis, diagnosis facilitates treatment, and treatment facilitates profit. Introducing a new mental disorder, like PTSD in DSM-III for example, creates an opportunity for the medical industry to benefit from making diagnoses - that is, from making people sick. 
Medical students will often "look" at patients as well, sitting in on diagnostic interviews in order to prepare for diagnosing their own patients, once certified. The spectacle of the diagnostic interview may prove harmful, as patients could feel uncomfortable answering a laundry list of questions as the psychiatrist and medical student take notes on a notepad, check off boxes next to symptoms, and scribble codes on insurance claim forms. The presence of another medical professional, the medical student, whose sole purpose in being there is to observe and learn for future practicethereby ensuring the continuance of psychiatry and psychiatric "expertise"-may exacerbate the potential for harm. The diagnostic process implies that advancing medical knowledge is more important than comforting patients. When a rape survivor, for example, is screened for PTSD, she is put on display for the doctor and her $\leftrightarrow$ his students so that they can diagnose her, but also so that medicine can generate more knowledge about trauma. The diagnostic interview may retraumatize that survivor. The medical gaze, vis-a-vis the diagnostic interview, puts the individual front and center while also stripping her $\leftrightarrow$ him of an identity outside of that for which she $\leftrightarrow$ he is being treated. As we consider whose interests are served in the diagnostic interview, we might wonder if medicine is actually making people sick for its own self-serving agenda. This speculation should not suggest that medical professionals themselves are consciously making people sick in order to profit, nor should it imply a simplistic "good guy" (patients) and "bad guy" (doctors) playing field. Rather, the mechanism of the medical gaze and the colonization of traumatized patients function in tandem to produce certain effects (e.g., making a $D S M$ diagnosis leads to developing a treatment plan), regardless of its stakeholders' intentions. 
Speculating about whether the medical gaze is "a tacit form of violence, all the more abusive for its silence, upon a sick body that demands to be comforted, not displayed," Foucault questions the very ethics of modern medicine (Birth 84). "Can pain be a spectacle?" he asks (ibid). Foucault's speculation begs yet another question: whether $D S M$ is not also "a tacit form of violence." Psychiatrists, for instance, "look" at patients, attempting to see through the surface reality, the stories and psychological symptoms with which patients present, to the root of that distress, a mental disorder. As explicated above, the diagnostic process alone can inflict violence as it functions to facilitate doctors' and not patients' understanding of how the psyche works.

Despite its propensity for violence, modern medicine is often touted as a benchmark of human advancement as a species. Medical research can and does reveal phenomenon previously unknown (and thus untreatable) about the body. The gaze enables medical knowledge to continue indefinitely, which can improve the quality of and extend people's lives. From this perspective, the diagnostic interview and the DSM manual itself are signs of progress. For example, DSM has been crucial in helping to recognize catastrophic experiences as traumatic. For survivors of rape who may struggle with guilt and self-blame, a PTSD diagnosis can assist in reversing shame. PTSD labels sexual violence traumatic, a disease of sorts, complicating the myths that victims can prevent rape if they really want to, and that victims deserve to be raped when they dress or act in certain ways, among other pervasive rape culture ideologies that can lead survivors to blame themselves. The myth that someone cannot be raped by a familiar with whom they have previously consented to sexual relations, for example, can lead survivors to believe that rape did not really happen, that it was all in their heads. The 
diagnosis of PTSD can facilitate recognition of rape as an act of power and control, of violence, and can challenge the very exploitation of power that produces, enables, and sustains rape. Medicine may be making us sick(er), but it may also be waking us up to truths that can reverse harmful myths and heal us. Analyzing the discursive construction of trauma in DSM-5 points up this duality. Yet as my analysis in the next sections suggest, this duality may prove to be just another mechanism through which modern medicine benefits from human suffering.

\section{The Social Construction of Mental Illness}

$D S M-5$ is the result of more than twelve years of work and upwards of $\$ 20$ million. Revising DSM was a costly endeavor. The investments of money and time that went into producing DSM-5 suggest that the manual has immense power to shape how we understand suffering. I argue that the manual's processes of production expose mental illness as, by and large, a Western social construct. As I aim to show in this section, the very production of $D S M-5$ regulates whose knowledge about mental illness counts as knowledge, and what mental illness even means.

The content and structure of $D S M-5$ are determined by institutions working to medicalize mental healthcare: chiefly the American Psychiatric Association (APA), the largest psychiatric association in the world. ${ }^{11}$ This latest edition of the manual was collaboratively revised and authored by "leading authorities on mental disorders in the world"- mostly white men ${ }^{12}$ — organized into thirteen working groups supervised by the

\footnotetext{
${ }^{11}$ While APA is the largest psychiatric association in the world, it is comprised primarily of members based in the United States.

12 The DSM-5 Task Force, for example, was chaired by two men (David J. Kupfer, M.D. and Darrel A. Regier, M.D., M.P.H.) and made up of roughly $27 \%$ women and approximately $73 \%$ men, the majority of
} 
DSM-5 Task Force, the APA Board of Trustees, and other review bodies (DSM-5 xliii). In "collectively represent[ing] the global expertise" of mental healthcare, the authors' status endows $D S M-5$ with global authority (ibid). While $D S M-5$ is designed for clinical use across the globe, its authors conducted field trials to assess the "reliability, feasibility, and clinical utility" of recommended revisions at medical-academic sites in North America exclusively ( $D S M-58)$. Reviews from outside experts and to a lesser degree, the public, shaped and determined what and how recommended revisions were implemented. However, it is important to note that public input was minimal as the DSM-5 Task Force allowed only two months, barely $2 \%$ of the total time spent revising the manual, for the public to post feedback on recommended revisions, on a website not readily accessible. $D S M-5$ 's notoriety as the gold standard for mental healthcare worldwide, shadowed by its primarily American, white, male authors, suggests that the United States and the ideological system of white patriarchy regulate whose knowledge about the mind, emotions, and behaviors counts as expertise; and moreover, suggests that psychiatry is fundamentally a Western interpretation of so-called "mental health."

$D S M-5$ is governed by clinical utility as both a practice, making diagnoses, and an ideology, a normative vision of diagnosis and intervention as the most important goals in psychiatric settings. A common, yet contested concept in the medical field, clinical utility refers to "the usefulness of an intervention for, or in, clinical practice. Utility is also associated with utilitarianism - that is, the ethical doctrine of achieving the greatest good for the greatest number" (Smart 1). Basically, DSM-5's primary purpose is to not only medicalize mental healthcare, but to universalize it - "to achieve the greatest good for the only three of whom were women. For a complete list of DSM-5 authors and consultants, refer to the manual's introductory pages. 
greatest number"- through establishing a common classification system for psychological suffering. However, the hegemony embedded in and enacted through DSM-5 may undermine the manual's goal of clinical utility.

The production of $D S M-5$ raises many questions that point up the manual's foundations in racialized, gendered colonial ideologies. How does the manual account for the many people suffering from PTSD (and other DSM-5 disorders) who cannot afford or do not have access to medical care? Whose experiences may not be represented in the research psychiatrists use to generate knowledge about PTSD? How does the manual integrate non-medical knowledges of patients and those who care for them, such as alternative healthcare practitioners, social workers, and legal advocates, many of whom must utilize $D S M-5$ criteria even as they are not qualified to make diagnoses? How does the APA determine "expertise," particularly as its notion of expertise largely occludes (or renders mere commentary) patients' expert knowledge of their own embodied experiences? How can North American medical sites alone sufficiently assess revisions that will impact practitioners and patients across the globe? I pose these questions not to suggest that they can be answered here, but to emphasize that knowledge about mental illness is socially constructed, and that this construct (i.e., DSM-5) serves a particular agenda and a particular power structure. The manual serves racialized, gendered Western colonial interests and arrangements of power. Excluding or minimizing input from outside of itself (i.e., outside of the APA), DSM-5 reinforces its own ideologies more than it develops knowledge about and effective treatments for mental disorders.

Following the scientific imperative to explicitly disclose one's research methodology, the APA discloses its DSM-5 revision guidelines outright. However, a 
closer analysis reveals gaps and contradictions pervading their methodology, suggesting that the results of their research may also be biased and contradictory. In DSM-5's introduction, the APA explains the revision process in detail. In revising DSM for the fifth edition, the working groups followed four basic principles. The first and second principles reify $D S M-5$ as an exclusively clinical tool. They state that since $D S M$ is intended primarily for clinical use, (1) "revisions must be feasible for routine clinical practice," and that (2) researched evidence must drive any recommended revisions (DSM5 7). In other words, revision recommendations had to be tested in clinical settings before going into print. Researched evidence, then, came strictly from contexts in which mental health patients were already being treated through Western interventions. The clinical utility mandate left no room for research about mental health in non-medical cultural settings, such as communities collectively remembering, mourning, trying to make meaning of, and healing from traumatic events. ${ }^{13}$

The third and fourth revision principles regulate the degree of change from the fourth to fifth editions of DSM. They state that authors must, at once, (3) maintain continuity with previous editions wherever they can, while also placing (4) "no a priori constraints... on the degree of change between DSM-IV and DSM-5" (DSM-5 7). These last two principles seem to contradict one another. Maintaining as much continuity as possible $i$ a a priori constraint, restricting the extent to which the manual's authors could research $D S M$ disorders in new, non-biomedical ways (e.g., in non-clinical

\footnotetext{
13 A few examples of communities working to memorialize and help people heal from traumatic events include The Center for Victims of Torture, an international nonprofit organization that advocates for victims of torture and violent conflict; INCITE!, a national network of "radical feminists of color working to end violence against women, gender non-conforming, and trans people of color, and our communities" in the United States; and, The Mennonite Central Committee, an Anabaptist nonprofit organization that provides relief, development and peace to those in need around the world (“Who is INCITE?").
} 
settings). If the permissible range for change runs from zero (maintaining total continuity) to infinity (imposing no a priori constraints), we may conclude that mental illness itself is a spectrum. The spectrum, in turn, may fuel the notion that psychiatry is not a legitimate science, which could easily result in the APA trying to stabilize categories of mental illness even as those categories fluctuate (e.g., homosexuality was originally classified as a mental disorder, $D S M-I$ and $D S M-I I$, before it was removed in $D S M-I I I)$. The very revision process is a cycle that can never be resolved so long as DSM remains a primarily clinical text. To break the cycle—-for example, to actively solicit contributions from non-medical settings and non- so-called experts-may also break the hold $D S M$ has on global mental healthcare, potentially rendering the manual outmoded or altogether obsolete. DSM-5 stands to lose significant credibility if it seeks too far outside of its predecessors, but also if it does not allow itself free reign to do so. In regulating and deregulating change in this way, the third and fourth revision principles pay lip service to the impermanence of mental disorders, but ultimately work to keep DSM relevant and to thereby ensure its survival into the future.

$D S M-5$ discourse shapes medical and also sociocultural understandings of mental illness. While clinical in scope, DSM-5 shapes how we understand the psycheespecially the way we understand mental health as distinct from physiological, social, and other forms of health. In Crazy Like Us: The Globalization of the American Psyche, American journalist Ethan Watters claims that the United States is "homogenizing the way the world goes mad" (2). $D S M-5$, in functioning as the global authority on mental disorders, shapes definitions and prognoses of, as well as treatments for, so-called mental illnesses. As Watters explains, 
Our golden arches do not represent our most troubling impact on other cultures; rather, it is how we are flattening the landscape of the human psyche itself. We are engaged in the grand project of Americanizing the world's understanding of the human mind... Our definitions and treatments have become the international standards... It turns out that how a people in a culture think about mental illnesses - how they categorize and prioritize the symptoms, attempt to heal them, and set expectations for their course and outcome-influences the diseases themselves. (1-2)

In short, Watters' claim here suggests that how we think about the mind shapes the mind itself. Throughout the book, Watters case studies the evolution of DSM disorders such as anorexia, PTSD, schizophrenia, and depression in Hong Kong, Sri Lanka, Zanzibar, Japan, and other non-Western global settings. As the U.S. constructs and universalizes knowledge about mental illnesses, populations absorb DSM classifications. Under the influence of Western medical knowledge, clinicians increasingly attribute symptoms such as insomnia to anxiety disorders, poor appetites to depression, nightmares to PTSD, and so forth. Put simply, DSM-5 may (partially) produce the very diseases it purports to simply describe, the very subjects it aims to make well, and the very embodied experiences of illness it fails to acknowledge. Once again, the homogenization of madness may say more about protecting and advancing the interests of medical institutions than of caring for patients.

The circulation of DSM-5 in professional and public contexts further illustrates how the manual shapes sociocultural understandings of mental illness. While aiming to treat people suffering globally, it takes a narrow (biomedical) approach to suffering, 
which represents an intensification of medicalizing trauma by way of "new and complex, usually techno-scientifically enmeshed ways" (Clarke et al. 47). Moreover, given the wide variation of "social, psychological, and biological factors that may have contributed to [a patient] developing a given mental disorder," diagnostic reliability seems an unproductive, even improbable goal (DSM-5 19). The manual may function less as a tool for providing better healthcare, and more for generating profit for the psychiatric industry (e.g., a diagnosed disorder necessitating medical interventions, like therapy or medications, puts more money into doctors' pockets, rendering and keeping patients dependent upon them).

While $D S M-5$ 's cost is prohibitive—around $\$ 100$ for paperback and $\$ 150$ for hardcover copies, potentially limiting its reach to those who can afford it such as medical practitioners, insurance companies, and others required to use the manual for professional or research purposes - the manual is publically available in bookstores, on Amazon, through other online booksellers, and through the American Psychiatric Association directly. You can even buy DSM-5 at Walmart. Returning to Watters' logic, the manual's appeal to both clinical (expert) and public (layperson) audiences exposes the insidiousness of $D S M$ discourse. Moreover, its ready availability may indicate the pervasive self-help culture in the United States - a trend of individualizing suffering, interpreting so-called negative feelings as indicative of some disorder or dysfunction, and putting mental health discourse (i.e., $D S M-5$ ) into the hands of the public. In this way, the APA ensures profits for itself, not only through selling copies of the manual but also through inculcating people into mental healthcare jargon, into the mental healthcare system of diagnosis and treatment itself. 


\section{"The medical bipolarity of the normal and the pathological"14}

Modern Western medicine demarcates so-called "normal" (healthy) from "pathological” (diseased) subjects. Producing new subjects and new experiences of subjectivity, DSM-5 normalizes individuals who suffer minimally or not at all and pathologizes individuals who suffer intensely as people internalize the manual's diagnostic categories. Populations internalize and increasingly manifest symptoms that align with $D S M$ diagnoses ("Major Depression”). For example, PTSD produces pathological subjects permanently marked by having survived (a) traumatic event(s), who in being diagnosed with PTSD, may come to actually experience their reactions to such events as abnormal, maladaptive, and dysfunctional. Producing that which it seeks to diagnose, the mental patient, DSM-5 normalizes subjects to pathologize others, pathologizes subjects to normalize others.

In the case of traumatic disorders in particular, DSM-5 constructs normal and pathological responses to traumatic events, which the manual defines as events involving "actual or threatened death, serious injury, or sexual violence" (DSM-5 271). Trauma is rendered abnormal in DSM-5 and in previous editions of the manual, "outside the range of usual human experience." This conceptualization of trauma assumes a universal understanding of what is traumatic (pathological) and what is usual (healthy). The very inclusion of traumatic disorders in DSM-5 renders trauma pathological. To be sure, some survivors may develop PTSD due to genetic, biological, environmental, or experiential predispositions to mental illness. Similarly, many trauma survivors never develop PTSD, or only experience its symptoms short-term. Whatever the reasons why a patient does or

\footnotetext{
${ }^{14}$ This phrase comes from Michel Foucault's Birth of the Clinic and describes the ideological shift in medicine, from a model of health to a model of normalcy (35).
} 
does not develop PTSD, survivors who suffer in the wake of traumatic events are pathologized by DSM-5's inclusion of PTSD as a mental disorder.

We are enmeshed in a kind of trauma culture, experiencing first-hand or witnessing "death, serious injury, or sexual violence" on a regular basis. We regularly encounter domestic battery, sexual assault, poverty, and other (potentially) traumatizing experiences, suggesting a kind of normality to death, injury, and violence. As reflected in changes made to the classification of traumatic disorders in DSM-5, Western society could be described as obsessed with trauma, increasingly curious about how trauma impacts not only combat veterans, first responders, and others living or working in war zones, but also children and adults in their very own homes. Traumatic disorders were among the most significantly revised group of disorders in DSM-5. The manual features an entirely new chapter, "Trauma- and Stressor-Related Disorders," as trauma "is increasingly at the center of public as well as professional discussion," marking a perceptual shift in understanding trauma as a distinct and pervasive illness ("PTSD" 1). $D S M-5$ pathologizes deathly, injurious, violent events, while trauma culture normalizes such events. This contradiction constructs trauma as intrinsic to the human condition (normal), yet also distressing (pathological), thereby producing a chronic dependence upon the manual's "expert knowledge" of psychological trauma even if and when "trauma" may not be the most efficacious or appropriate means of understanding suffering.

In this newly-added chapter on trauma and stressor-related disorders, the manual describes several "disorders in which exposure to a traumatic event is listed explicitly as a diagnostic criterion" (DSM-5 265). Firstly, Reactive Attachment Disorder: relatively 
rare, this diagnosis applies to infants or children whose basic needs are not met and who fail to form healthy attachments with caregivers. Secondly, Disinhibited Social Engagement Disorder: characterized by culturally inappropriate or overly familiar behavior with strangers, this diagnosis describes a child who "violates the social boundaries of a culture" often due to neglect (ibid). Thirdly, Posttraumatic Stress Disorder (PTSD): first featured in DSM-3 (released in 1980), PTSD affects people of all ages, particularly those older than six years of age, and reflects a prolonged disturbance in an individual's life after having been exposed to traumatic events. Distinguishing these three conditions as distinct $D S M-5$ disorders may indicate an evolving understanding of how much trauma actually exists. Trauma impacts populations in the public spotlight (e.g., Vietnam War veterans) as well as those marginalized and rendered invisible by traumas occurring behind closed doors (e.g., victims of intimate partner violence). Moreover, the development of these disorders illustrates how traumatic events ranging from abuse and neglect to torture and homicide impact people differently depending on age, family background, cultural affiliations, gender, and other naturalized identity markers.

Despite the chapter's presentation of trauma as a diverse cluster of disorders with wide-ranging symptom variations, $D S M-5$ ultimately regulates the extent to which symptoms signify disorder. For example, the fifth edition includes extensively revised PTSD symptoms which aim to classify PTSD as a traumatic and no longer an anxiety disorder, as it had been classified in DSM-III and DSM-IV. First and foremost, the new criteria set stricter boundaries around what a traumatic event is, the trigger of PTSD, endowing clinicians with the authority to determine whether or not a patient is 
traumatized. Traumatic events now specifically include sexual violence as well as "recurring exposure that could apply to police officers or first responders" ("PTSD"1). This change distinguishes sexual violence as a unique kind of trauma in that it does not necessarily involve actual or threatened death, which characterize other kinds of traumatic events. While distinguishing sexual violence and first-responder exposure as unique forms of traumatic events works to validate the traumatic experiences of sexual violence survivors and first responders, the general move to clinically regulate traumatic events may inadvertently barr from treatment patients whose experiences do not fit neatly inside the $D S M-5$ conception of such events - such as the disorientation, hypervigilance, and self-loathing that emotional abuse can induce.

The revised definition of trauma also omits "[1]anguage stipulating an individual's response to the event—intense fear, hopelessness, or horror, according to $D S M-I V \ldots$ because that criterion proved to have no utility in predicting the onset of PTSD" ("PTSD" 1). A patient's emotional experience has "no clinical utility," in other words. Behavior, observable and trackable by virtue of the medical gaze, is deemed more clinically useful. This behavioral bias puts clinicians in a position of power over patients, the power to interpret their personal responses to traumatic events, discrediting patient testimonies as incomplete, or even entirely inaccurate. $D S M-5$ 's very barometer for assessing mental dysfunctioning reduces the complexity of trauma by focusing on behavior in social and occupational activities when, in fact, trauma may be more apparent in a patient's daily habits including, but also beyond, social and occupational situations.

Furthermore, $D S M-5$ stipulates that in order for "actual or threatened death, serious injury, or sexual violence" to count as traumatic, the event must have been violent 
or accidental" (DSM-5 271). One can easily interpret from here that losing a loved one to a diagnosed disease (e.g., cancer) is not traumatic because death from diagnosed cancer is predictable and not inherently violent. The reclassification of PTSD as an event- and not anxiety-based disorder, coupled with the stricter delineation of traumatic from nontraumatic events, locates the roots of dysfunction outside of the individual patient (i.e., in the event). Yet any classification of PTSD in a manual of mental disorders also pathologizes the survivor by labeling her $\leftrightarrow$ his response to traumatic events as dysfunctional. This message is contradictory. If the event is traumatic, then the patient is traumatized. This would suggest that anyone who survives certain events is traumatized. Yet DSM-5's symptom categories for PTSD, analyzed in the upcoming sections, are described in relation to traumatized people.

In DSM-5, life-threatening, life-ending, injurious, or sexually-violent traumatic events produce psychological effects. The APA writes, "[ $\mathrm{t}]$ he essential feature of posttraumatic stress disorder (PTSD) is the development of characteristic symptoms following exposure to one or more traumatic events" (DSM-5 274). Events produce PTSD. The manual discursively constructs trauma as an event, yet then constructs its symptoms as individual pathology. In other DSM disorders, however, mental dysfunctions produce disorders. As discussed at the beginning of this chapter, the manual's definition of mental disorder is "a syndrome characterized by clinically significant disturbance in an individual's cognition, emotion regulation, or behavior that reflects a dysfunction... underlying mental functioning" (emphasis added, DSM-5 20). We can conclude, then, that in arising from certain kinds of events, PTSD actually 
falls outside of the DSM-5 definition of disorder. Perhaps trauma does not belong in the manual at all.

PTSD may also prove a controversial category for inclusion in DSM-5 because the manual takes a remedial rather than preventative approach to trauma. The DSM-5 description of PTSD is constructed around its effects on individuals, not whole communities or populations, even as traumatic events are often shared experiences. Across the globe, combat veterans engage in and witness the killing of innocent people; girls and women endure daily sexual violence; and, first responders provide relief aid to thousands of people in the wake of earthquakes and other natural disasters. But DSM-5's assertion that PTSD (like other mental disorders) arises from mental dysfunctioning locates the root of trauma in individuals and more specifically, those individuals suffering from, rather than perpetrating, trauma. Using mental dysfunction to describe the root of PTSD may shame victims for having endured traumatic events, and for struggling to function in their wake. $D S M-5$ describes trauma as an event, yet uses trauma as a vehicle for understanding individuals' behavioral and social activities in the wake of those events, not for understanding what causes those events to occur in the first place.

Conceptualizing trauma through the individual psyche produces many effects.

Firstly, conceptualizing trauma psychologically individualizes and pathologizes traumatic experience; traumatic events are daily realities as well as one-time catastrophes (e.g., 911 or the bombing of Hiroshima), and can include ongoing neglect, abuse and other forms of domestic violence. To psychologize trauma, vis-a-vis the diagnosis of PTSD, implies that trauma is not a daily reality but a psychological dysfunction. To be sure, trauma survivors experience traumatic events in a wide variety of ways, whether those 
events are acts of interpersonal or collective violence, depending upon familial, social and cultural norms. As DSM-5 attests, "[t]hresholds of tolerance for specific symptoms or behaviors differ across cultures, social settings, and families. Hence, the level at which an experience becomes problematic or pathological will differ" (14). In some settings, terrorist attacks may be such regular occurrences, the population has adapted to integrate this kind of event as "normal" or "usual." DSM-5 acknowledges cultural variation, yet seeks to universalize its discourse, exposing trauma as both pathological, contextspecific, and normal, global.

$D S M$ is designed to diagnose individuals, not communities or group populations. Yet members of oppressed groups are more vulnerable to traumatic stress surrounding violence in their communities or among their social groups - for example, Hurricane Katrina's disproportionate displacement effects on populations in New Orleans already contending with poverty and other social disadvantages. Further, trauma can be generational, as is evident in epigenetic accounts of Nazi Holocaust survivors' children and grandchildren. ${ }^{15}$ The individualization of suffering decontextualizes trauma, at the same time as the manual acknowledges the realities of group variation (cultural, social, familial). To pathologize trauma responses, to render them dysfunctional by diagnosing patients with PTSD, normalizes (i.e., renders functional) individuals who function as they did before the traumatic event(s). The bifurcation of those who struggle to function (i.e., survivors with PTSD) and those who function "normally" (i.e., survivors without PTSD) can retraumatize patients who are most vulnerable to developing PTSD symptoms, and can glorify those who live as if the event(s) never happened.

\footnotetext{
${ }^{15}$ For more on the transgenerational effects of trauma, see Tania L. Roth's "How Traumatic Experiences Leave Their Signature on the Genome: An Overview of Epigenetic Pathways in PTSD," and Judith Shulevitz's "This is the Science of Survival."
} 
Secondly, conceptualizing trauma through the individual psyche puts the clinician, not the patient, in the position of determining how an event has affected a survivor, as well as whether or not that event even counts as traumatic. By DSM-5 standards, a traumatic event is one that produces PTSD. Period. The APA surveils trauma. Moreover, by stating that exposure to trauma through "electronic media, television, movies, or pictures" does not count "unless this exposure is work related," DSM-5 leaves out people who experience PTSD symptoms through traumatic media exposure outside of work. This exclusion further implies that work is a more legitimate (i.e., clinically useful) context for assessing symptoms than other contexts in which obligation or coercion may expose someone, against her $\leftrightarrow$ his will, to traumatic media. For example, this exclusion leaves out someone forced to watch violent pornography by her $\leftrightarrow$ his partner or spouse. The "only work-related" disclaimer assumes that a sense of traumatizing obligation can only come from occupational settings, an omission that is especially risky in situations of intimate captivity in which someone is held against their will by someone they know, someone with whom they may have already entered into a consensual relationship.

Thirdly, conceptualizing trauma through the individual psyche centralizes the aftermath of traumatic events rather than events themselves, or even preceding circumstances that may have produced such events. The manual organizes PTSD symptoms into eight symptom clusters: exposure, intrusion, avoidance, negativity, and arousal. These clusters stipulate that an individual must first be exposed to a traumatic event or events (Criterion 1) and then reexperience those events in intrusive, debilitating ways (Criterion 2-5). Further, the manual stipulates that a patient must experience 
symptoms for more than one month (Criterion 6) and, as a result, experience "clinically significant distress or impairment in social, occupational, or other important areas of functioning" (Criterion 7) (272). These seven criteria centralize trauma's aftermath, its effects rather than its causes.

Finally, practitioners can only count these symptoms as indicative of PTSD if they are "not attributable to the physiological effects of a substance (e.g., medication, alcohol) or another medical condition" (Criterion 8) (ibid). All eight criterion place importance on how an individual survives traumatic events, deemphasizing how the sociocultural settings in which traumatic events occur may impact one's ability to survive. To approach trauma as an afterthought in this way, the manual may inadvertently obscure the traumatic nature of certain events themselves (e.g., war, by nature, involves violence and death), or overlook measures that might prevent some traumatic events from occurring in the first place (e.g., educating teenagers about dating violence to prevent domestic abuse in adulthood).

To demonstrate how these eight PTSD criterion discursively construct trauma, and to expose gaps and contradictions within this discursive construction, I analyze each symptom cluster in depth, renaming each cluster by placing the adjective "traumatic" before the symptom name (e.g., exposure to trauma becomes "traumatic exposure"). This renaming works to problematize the $D S M-5$ assumption that exposure, intrusion, avoidance, negativity, and arousal symptoms inherently signify trauma, and to suggest that they may be more efficacious in diagnosing PTSD if they are explicitly contextualized by trauma. That is, I literally "traumatize the symptoms." 
I also analyze the diagnostic questions associated with each set of symptoms. These questions make up the diagnostic interview: a question and answer session between clinician and patient to assess the possible presence of a mental illness. As Abraham M. Nussbaum, M.D., author of The Pocket Guide to DSM-5: The Diagnostic Exam explains, the diagnostic interview should be about forming "a therapeutic alliance" rather than assessing psychiatric symptoms... in an effort to "instill hope and provide support to a demoralized person" (20). However, as my analysis suggests, the diagnostic questions associated with PTSD symptoms may not actually fulfill the stated goals of a diagnostic interview. Deconstructing each symptom cluster and its accompanying diagnostic questions lays the foundation for the discursive analyses that comprise the rest of this dissertation. To put DSM-5 nosology to the test - to reveal how closely it converges and diverges from literary and cinematic constructions of trauma analyzed in subsequent chapters - requires a thorough analysis of each symptom cluster and its accompanying diagnostic questions.

\section{Traumatic Exposure}

In order for a patient to be diagnosed with PTSD, she $\leftrightarrow$ he must first experience traumatic exposure. Labeled "Criterion A," this symptom set refers to direct or indirect “exposure to war as a combatant or civilian, threatened or actual physical assault... threatened or actual sexual violence... being kidnapped, being taken hostage, terrorist attack, torture, incarceration as a prisoner of war, natural or human-made disasters, and severe motor vehicle accidents" (emphasis added, DSM-5 274). Exposure may be one-time only, as in the case of a severe earthquake, or repeated and ongoing, as in the 
case of being a prisoner of war. Traumatic exposure means a direct experiencing or live witnessing of singular or ongoing death, violence, or serious injury.

By itself, the noun "exposure" has multiple meanings that can extend DSM-5's limited conception of exposure as a one-off experience of death, violence, or injury: (1) an act of uncovering or leaving without shelter or defense, (2) the state of being subjected to an external influence, (3) an act of abandoning, and (4) the act of bringing something discreditable to light ("Exposure"). Combining the DSM-5 and Oxford English Dictionary definitions of exposure can help us understand traumatic exposure as experiencing or witnessing an event beyond one's control—one that leaves a person at the mercy of that event as if chaos (a lack of control) is inherently traumatic. Traumatic exposure signals defenselessness, vulnerability, and victimization. It sets off all subsequent PTSD symptoms: intrusive memories of (Criterion B), avoidance of associations with (Criterion C), negative thoughts and feelings around (Criterion D), and changes in reactions to the original traumatic event (Criterion E). The sequence of these criteria suggests that the event itself, not individual functioning, is most central to making a PTSD diagnosis, and that all other symptoms arise from that event rather than mental dysfunctioning.

We can further understand traumatic exposure as revealing something shameful. In the very act of embodying exposure symptoms, a patient diagnosed with PTSD exposes the atrocity she $\leftrightarrow$ he has witnessed, thereby making that atrocity visible - more possible to confront and change. A rape survivor screened for PTSD, for instance, brings to light the ruinous consequences of sexual violence that can continue well beyond "the event" and throughout a survivor's lifetime. In this way, traumatic exposure at once 
describes the individual survivor as well as the brutality of the external world, exposing mental disorders as more complex than the DSM-5 definition of them suggests in its stipulation that they are about mental dysfunction.

To assess traumatic exposure, clinicians ask two initial screening questions at the beginning of the diagnostic interview: "What is the worst thing that has ever happened to you? Have you ever experienced or witnessed an event in which you were seriously injured or your life was in danger, or you thought you were going to be seriously injured or endangered?" (Nussbaum 90). As the initial ones asked, these questions may not facilitate a therapeutic alliance and may actually silence or even retraumatize patients. They assume a level of trust that cannot be reasonably expected from patients within the first few minutes of an initial therapy session. Patients may need more time to feel safe, to open up. In addition, read together, these questions assume a shared understanding of "worst" experience as a life-threatening one, which is the APA's and not necessarily the patient's point of view, thereby endowing the clinician with the power to decide if the event was traumatic and therefore, whether to even proceed with screening for PTSD.

Furthermore, the premise of these questions is contradictory. On the one hand, they target the issue at hand, signs of traumatic exposure. On the other hand, they assume a presence of mind, an ability to recall events and to identify them as "the worst thing," when trauma invariably alters cognition. Patients may have no language for what they have been through, particularly as neuroscience is finding that "all trauma is preverbal... [that] [t]rauma by nature drives us to the edge of comprehension, cutting us off from language based on common experience or an imaginable past" (van der Kolk 43). Trauma can distort not only language and memory, but even one's perception of what happened 
as traumatic. For example, a kidnapping survivor can develop Stockholm Syndrome, a positive emotional identification with her $\leftrightarrow$ his captor that can result in the victim actually defending the kidnapper. Stockholm Syndrome would potentially change the victim's perception of the trauma in such a way that she $\leftrightarrow$ he does not even recognize kidnapping as "the worst thing," as dangerous, as injurious. Given the assumptions of trust and cognitive coherence underlying these screening questions, they may prove ineffective in determining whether or not a patient has traumatic exposure.

\section{Traumatic Intrusion}

After determining that a patient has traumatic exposure, clinicians then attempt to determine how a patient may reexperience the event(s) afterwards through traumatic intrusion. Labeled "Criterion B," this symptom set refers to "recurrent, involuntary, and intrusive recollections of the event" which "are distinguished from depressive rumination in that they apply to involuntary and intrusive distressing memories. The emphasis is on recurrent memories of the event $[(\mathrm{s})]$ that usually include sensory, emotional, or physiological behavioral components" (emphasis added, DSM-5 275). Traumatic intrusion means an unwanted, repeated reexperiencing of trauma through mind and body.

By itself, the noun "intrusion" has multiple meanings that can extend DSM-5's conception of intrusion as strictly an individual experience: (1) the action of unwelcomed thrusting or forcing in, (2) "violent or unjust entrance into or seizure of land or rights belonging to another," and (3) the act of introducing something inappropriately ("Intrusion"). Combining the DSM-5 and Oxford English Dictionary definitions of intrusion can help us understand traumatic intrusion as a hostile takeover. This takeover 
interferes with meeting core needs, including physiological needs like sleep, safety needs like freedom from fear, belongingness needs like intimacy, and self-actualization needs like personal growth. ${ }^{16}$ Thus, traumatic intrusion is characterized by the traumatic events themselves wielding power and control over a survivor.

We can further understand traumatic intrusion as a breakdown of autonomy, given that the event(s) continue(s) to play out in a survivor's life long-after it has/they have passed. However, DSM-5's traumatic intrusion symptoms assume that trauma is finite, with fixed beginning and end points, as the focus is on memories of events, which suggest that they are now in the past. In fact, trauma is in many ways infinite. For example, while the event that was "9-11" is over and hijacked planes are no longer crashing into the World Trade Centers or the Pentagon, people across the globe experience grief, poverty, dislocation, war, environmental hazards, and fear as a result of 9-11. Indeed, traumatic intrusion can describe not only symptoms an individual may experience as a result of surviving trauma, but also the very ongoing and insidious nature of trauma itself.

To assess patients' experiences of traumatic intrusion symptoms, clinicians inquire into how a patient remembers and feels ${ }^{17}$ about the event(s) — specifically, through five questions about memory, dreams, flashbacks, exposure distress, and physiological distress: "After that [traumatic] experience, did you ever experience intrusive memories of the experience when you did not want to think about it?... Did you have recurrent, distressing dreams related to the experience?... After that experience, did you ever feel like it was happening to you again, like in a flashback where the event is

\footnotetext{
16 These needs are part of Maslow's Hierarchy of Needs, a five-stage model of basic through growth needs. ${ }^{17}$ Note that even though DSM-5 excluded emotional responses from PTSD diagnostic criteria "because that criterion proved to have no utility in predicting the onset of PTSD," the manual relies heavily upon emotional responses in assessing traumatic intrusion ("PTSD” 1).
} 
happening again?... When you are around people, places and objects that remind you of that experience, do you feel intense or prolonged distress?... When you think about or are around people, places, and objects that remind you of that experience, do you have distressing physical responses?" (Nussbaum 90-91). In order to be diagnosed with PTSD, a patient must answer at least one of these questions in the positive, exhibiting at least one of the five intrusion symptoms for one month or more after the event has passed. The structure of these questions may actually encourage patients to answer them in the positive, regardless of the degree to which they actually experience intrusion symptoms. To be sure, these questions may produce subjects who label their experiences in particular ways, planting seeds in a patient's mind that she↔he has experienced unwanted memories, dreams, and flashbacks; unwelcomed and overwhelming fear when she $\leftrightarrow$ he comes into contact with reminders of the traumatic event(s) - when the patient may not actually know.

Furthermore, even if the patient has experienced intrusion symptoms, the diagnostic questions assume a level of self-awareness on the part of the survivorpatient — an ability to connect intrusive memories, dreams, flashbacks, and/or emotional and physiological distress to the traumatic event(s) that prompted them. Traumatologist Dr. van der Kolk explains that the very nature of trauma can make cognitive, emotional and bodily presence difficult, if not impossible. As the very traumatic intrusion symptoms signify, trauma interferes with one's ability to stay in the present moment, thrusting her $\leftrightarrow$ him back into the past at the same time as reminding her $\leftrightarrow$ him that she $\leftrightarrow$ he has survived the event(s) into the future. Thus, the $D S M-5$ diagnostic questions for intrusion symptoms may prove ineffective. Distinguishing between past, present and 
future may also prove impossible for PTSD patients, thus making the accuracy of their answers to these questions questionable. Ironically, DSM-5 also invalidates patient testimonies by way of claiming that emotional responses to traumatic events are not clinically useful. Yet the APA's very recommendation to pose these traumatic intrusion questions in the diagnostic interview suggests that patient testimony is essential to diagnosing PTSD symptoms.

\section{Traumatic Avoidance}

After determining that a patient has been exposed to (a) traumatic event(s), and that she $\leftrightarrow$ he continues to reexperience the event(s) in some way, clinicians then examine how a patient may attempt to avoid associations with the trauma. Labeled "Criterion C," this symptom set refers to traumatic avoidance. Symptoms of traumatic avoidance include an avoidance of "[s]timuli associated with the trauma" or "deliberate efforts to avoid thoughts, memories, feelings, or talking about the traumatic event[(s)]" (DSM-5 275). Traumatic avoidance in DSM-5 indicates intentional efforts to avoid reexperiencing traumatic events through avoidance of internal or external triggers.

By itself, the noun "avoidance" has multiple meanings that can extend DSM-5's conception of avoidance as indicative of dysfunction: (1) an action of emptying a vessel; of clearing away, removal, ejection, excretion; (2) a means of emptying; the action of making void or of no effect; invalidation; annulment; and (3) the action of dismissing a person; the action of quitting, withdrawal, departure, exit ("Avoidance"). Combining the DSM-5 and Oxford English Dictionary definitions of avoidance can help us understand traumatic avoidance as conscious, strategic efforts to forget. These efforts might span 
from using drugs to numb pain, to moving to another region or ending relationships with other survivors of the trauma in order to remove from one's environment any reminders of the event(s). We can further understand traumatic avoidance as an agential attempt to recreate one's identity, post-traumatically (in the present). Traumatic events take on lives of their own. They may be so intolerable that in order to survive beyond them, one must actively work to erase all signs of the events having happened in the first place. As thoughts, memories, places, people, and other daily aspects of life associated with the trauma intrude upon a survivor's life in the present, he $\leftrightarrow$ she may go to great lengths to avoid them in order to avoid reexperiencing the horror of the original wounding.

Of course, total avoidance of traumatic stimuli is close to impossible as triggers are often both insidious and unpredictable, which may explain why DSM-5 describes avoidance symptoms as "efforts to avoid." A date rape survivor living in a college dormitory, for example, may end all communication with the perpetrator, avoid spending time with any mutual friends, and even move to a different part of campus. However, music, commercials and other mass media that feature men sexually exploiting women are so pervasive, the survivor may encounter a traumatic trigger simply by going to the grocery store and hearing a certain song play on their radio. Thus, traumatic avoidance is not so much about actual avoidance, but rather attempted avoidance.

$D S M-5$ 's conception of avoidance assumes a transparent link between traumatic stimuli and traumatic events when, in point of fact, there may be no obvious association between the two. A childhood incest survivor, for example, may present with a strong aversion to sharing a bed with his partner, yet have no memory of incest. He does not know why, he just knows that when he shares a bed with her, he cannot sleep. Insomnia 
has many forms, causes, and symptoms — only some of which pertain directly to trauma. The difficulty of linking traumatic stimuli and events may render traumatic avoidance an incomplete cluster of symptoms in DSM-5. Furthermore, this difficulty may render the very process of diagnosing PTSD ineffective. Returning to the recommended process for diagnosing traumatic intrusion symptoms discussed in the previous section (PTSD Criterion B) — in which clinicians ask patients about traumatic memories, dreams and flashbacks - how could a patient accurately answer those questions if she $\leftrightarrow$ he is experiencing avoidance to the point of forgetting events altogether?

In addition to espousing the erroneous belief that traumatic triggers and events clearly coincide, $D S M-5$ 's conception of avoidance also leaves out survivors who do not avoid trauma. Some survivors respond in opposite ways, drawn back to the original site of trauma and even to their perpetrators. Sigmund Freud described this tendency as the repetition compulsion, a "neurotic defense mechanism" in which a survivor seeks out the past trauma, over and over again, in an effort to rewrite the past (Diamond). Dr. van der Kolk debunks Freud's theory as "repetition leads only to further pain and self-hatred" (32). He reframes Freud's repetition compulsion as an addiction to trauma. Trauma changes body chemistry as "the body learns to adjust to all sorts of stimuli" (ibid). As Dr. van der Kolk explains, traumatized patients, who "often complain about a vague sense of emptiness and boredom when they are not angry, under duress, or involved in some dangerous activity," may use trauma like a drug (31). Providing "a sense of well-being and exhilaration," trauma may prompt a survivor "to crave the activity and experience withdrawal when it's not available" (32). The DSM-5 criterion for traumatic avoidance symptoms excludes any and all suggestions that trauma can produce pleasure, potentially 
excluding from PTSD diagnoses patients who present signs of craving, rather than avoiding, traumatic stimuli.

To assess patients' experiences of traumatic avoidance symptoms, clinicians ask: "Do you work hard to avoid thoughts, feelings, or physical sensations that bring up memories of this experience?... Do you work hard to avoid people, places, and objects that bring up memories of this experience?" (ibid). In order to be diagnosed with PTSD, a patient must exhibit at least one of the two avoidance symptoms, of internal or external reminders, for one month or more after the event has passed. By its own logic, DSM-5 represents psychological trauma as disorienting, fragmenting, and overwhelming. Yet the diagnostic questions for assessing traumatic avoidance assume a kind of self-coherency, on the part of the patient, that allows her $\leftrightarrow$ him to integrate stressors in the present with stressors of the past. The questions can only yield accurate answers if the patient has been able to remove herself $\leftrightarrow$ himself from the traumatic event(s) to such a degree that she $\leftrightarrow$ he can link internally- or externally-driven distress with the event(s), identify her $\leftrightarrow$ his efforts to avoid as "hard work," and recognize that struggle between remembering (and even actively seeking out trauma) and forgetting as a direct result of surviving trauma. The recommended diagnostic process for assessing traumatic avoidance symptoms may work best for eliminating PTSD as a possible diagnosis. That is, these questions may prove most appropriate for survivors who do not experience (significant) avoidance distress in the wake of trauma. 


\section{Traumatic Negativity}

After determining that a patient has been exposed to (a) traumatic event(s), that she $\leftrightarrow$ he continues to reexperience and actively avoids reminders of the event(s), clinicians then examine how trauma may impact the patient's cognition and mood. Labeled "Criterion D," this symptom set refers to traumatic negativity. Traumatic negativity impacts memory, self-esteem, mood, and social relationships. Symptoms include "negative alterations in cognitions or mood associated with the event [which] begin or worsen after exposure to the event" (DSM-5 275). Symptoms can manifest as an "Ii]nability to remember" something important about the event(s); "exaggerated negative beliefs or expectations about oneself, others, or the world;" a chronic "negative emotional state" accompanied by an "inability to experience positive emotions;" “diminished interest or participation in significant activities;" and a sense of “detachment or estrangement from others" (emphasis added, DSM-5 271-72). Traumatic negativity in DSM-5 signals cognitive, emotional deterioration that breaks down one's sense of connection with others as well as with oneself.

By itself, the noun "negativity" refers to "negativism," which has multiple meanings that can extend DSM-5's conception of negativity as something to offset by way of positive thinking, personal affirmations, redirecting, and other cognitive therapy techniques aimed at eradicating negative thinking, attitudes, behaviors, and relationships: (1) a habitual or behavioral tendency toward negativity, criticism, or skepticism accompanied by a failure to contribute something positive; (2) resistance to producing expected responses, oppositional behaviors or thoughts; and (3) outright antagonism, or a refusal to cooperate ("Negativism"). Combining the DSM-5 and Oxford English 
Dictionary definitions of negativity can help us understand traumatic negativity as an absence of presence, the absolute opposite of positivity which is characterized by presence - by affirmation and actuality. Absence may be measured by a variety of factors, including cognition and mood, and which fractures memory; erodes self-esteem and positive emotions; and which impedes and even severs one's relationships with others. Conceptualizing traumatic negativity through absence points up the sometimes deadly nature of trauma — not just in terms of the precipitating event(s), which may have directly involved death, but also in terms of how trauma can hollow one out, disintegrating one's perception of who she $\leftrightarrow$ he is, of how identity itself is created through our relationships with other people, places, events, activities, and other external sources of meaning-making.

We can further understand traumatic negativity as not only absence, but also distortion. Characterized by distortion, traumatic negativity interferes with one's ability to clearly associate traumatic stimuli with traumatic events, as described in preceding PTSD Criterion C, traumatic avoidance. A survivor suffering from traumatic negativity symptoms may have lost the ability to discern the causes of their suffering, compelled as she $\leftrightarrow$ he might be to view the situation as "all my fault." A patient expressing "exaggerated negative beliefs or expectations about oneself, others, or the world" may habitually blame herself $\leftrightarrow$ himself for the traumatic event(s); may resist or even refuse to see the event(s) from any other perspective. She $\leftrightarrow$ he has, in this way, been infected by the trauma as if it were a contagion whose sole function is to steal away hope, dignity, connection, purpose and in some cases, life itself. ${ }^{18}$ Conceptualizing trauma as a

\footnotetext{
${ }^{18}$ While statistics are inconclusive given that not all suicides are reported, not all suicides are actually intentional, and causes of suicide vary widely, there is strong evidence to suggest that people diagnosed
} 
contagion reveals how just as a leech latches onto other living bodies to suck blood, making itself stronger and the other weaker, traumatic events can latch onto survivors, slowly but steadily draining their very sense of being in the world. This leaching process has the effect of making the event(s) increasingly more important, in the mind of the survivor, and the survivor increasingly weaker.

To assess patients' experiences of traumatic negativity symptoms, clinicians inquire into seven different aspects of functioning: memory, self-image, attribution of responsibility, emotional state, social participation, social relationships, and ability to experience positive emotions. In order to be diagnosed with PTSD, a patient must exhibit dysfunction in at least two of these areas for at least one month after the event has passed. The recommended ways for clinicians to assess traumatic negativity may actually miss the mark. The accompanying diagnostic questions apply to other mental disorders as well—most notably, depressive disorders. While DSM-5 acknowledges the extensive overlaps among many mental disorders, and overlaps do not necessarily diminish key differences among disorders, the questions for assessing traumatic negativity may produce inconclusive answers for diagnosing someone with PTSD. Furthermore, the diagnostic questions may miss the mark because how they are constructed may produce unreliable answers. Given the sheer number of diagnostic questions associated with traumatic negativity symptoms and the homogenizing premises embedded therein, I will analyze each question separately and explain how each question may actually induce greater negativity.

with PTSD are at a higher risk of suicide: "PTSD alone out of six anxiety diagnoses was significantly associated with suicidal ideation or attempts" ("PTSD: National Center for PTSD"). 
To determine if the trauma has impaired one's memory, clinicians ask: "Do you have trouble remembering important parts of the experience?" (Nussbaum 91). This question assumes a universal understanding of what "important" means in the context of remembering trauma. In short, this question assumes that "the facts"- the who, what, when, where, why and how-are most important. Yet survivors frequently remember trauma in fragments, outside of the boundaries of time, outside of language itself. As the DSM-5's construction of PTSD symptoms illustrates, trauma survivors experience trauma after the fact —one month or more after the event(s) — rendering the "important parts" (the facts) less significant than the ways in which the facts (what happened) continue to affect a survivor in the present. Moreover, the allegedly inconsequential parts of the experience — sounds, smells, colors, tastes, and other sensory details—are often the most significant, the most remembered from a survivor's perspective. A rape survivor may recall in vivid detail the smell of the perpetrator's cologne, remembering little else of the experience. A combat veteran may repeatedly experience sounds like fireworks or popping balloons as gunshots. Who gets to say which parts of rape, war, or any other traumatic experience are important? This diagnostic question about memory suggests that the clinician, not the survivor, does. Shifting that power to the clinician, however wellintentioned she $\leftrightarrow$ he may be in helping the survivor, may further diminish the survivor's trust in her $\leftrightarrow$ his own experience, her $\leftrightarrow$ his own perception —a trust that is essential to restoring a sense of well-being in the world.

To determine if the trauma has impacted a survivor's self-image, clinicians ask: "Do you frequently think negative thoughts about yourself, other people, or the world?" (Nussbaum 91). This question could be on the list for diagnosing many other mental 
disorders besides traumatic ones. Nowhere is there an inquiry into how the traumatic event(s) may have induced negative thoughts. More importantly, the question lumps negative thoughts about oneself, others, or the world into one thought, which may lead to faulty answers. If a survivor thinks negatively about himself, but not others, he may answer in the negative since the majority of the question deals with thoughts about the external world ("other people, or the world"). If a survivor says that she thinks negatively about others but not herself, does her answer indicate PTSD or some other mental disorder, such as narcissistic personality disorder, which is characterized by an inflated sense of self and a devaluation of others? If witnesses to 9-11 think negatively about the world in general, but not themselves or others, does that mean they may have PTSD, or that they have developed a healthy attitude of caution about the world as a result of witnessing the mass destruction of the World Trade Centers? How does DSM-5 discern negative thoughts about oneself from thoughts about others or the world? Can we even separate these out? I pose these questions to illustrate how this diagnostic question raises more questions than it answers, potentially limiting the manual's prized goal of clinical utility.

To determine if trauma has skewed one's ability to attribute appropriate responsibility for the event(s), clinicians ask: "Do you frequently blame yourself or others for your experience, even when you know that you or they were not responsible?" (Nussbaum 91). This question literally consumes itself, rendering it virtually meaningless. If answered in the positive, the latter part of the question renders the misattribution of blame alluded to in the first part a conscious act of lying. Such a question assumes that the survivor already knows reality from fiction. That is, if a 
survivor displaces blame onto herself $\leftrightarrow$ himself or others when she $\leftrightarrow$ he "knows better" so to speak, then she $\leftrightarrow$ he may be perceived as playing games. In reality, a patient experiencing misplaced blame may need time to unravel the details and attribute responsibility more accurately. This diagnostic question may effectively blame the victim, and assumes that survivors can distinguish reality from projection: on the one hand, who/what is actually responsible for the event(s) and, on the other, the patient's (distorted or partial) version of events. This question can potentially debunk the entire premise of traumatic negativity symptoms.

To determine if the trauma has induced negative emotions, clinicians ask: "Do you stay down, angry, ashamed, or fearful most of the time?" (Nussbaum 91). Like a previous question ("Do you frequently think negative thoughts about yourself, other people, or the world?") and subsequent questions (analyzed next) for assessing traumatic negativity, this one also could be on the list for diagnosing many other mental disorders besides traumatic ones. Nowhere is there an inquiry into how the traumatic event(s) may have induced negative emotions. More importantly, this question lumps a range of socalled "negative" emotions — feeling down, angry, ashamed, fearful—into one thought, which may lead to faulty answers. If a survivor experiences one of these emotions but not others, she may answer in the negative. If a survivor feels one of these emotions but not others, and answers the question in the affirmative, does his answer indicate PTSD or some other mental illness, such as an anxiety disorder, which is characterized by fear? If a witness to sexual violence feels ashamed for not having stepped in to help, does that mean that she $\leftrightarrow$ he may have PTSD, or that she $\leftrightarrow$ he is like the millions of other bystanders who are too afraid to speak up, or too presumptuous about the goodness of 
others stepping in to help? How does DSM-5 discern staying down, from anger, from shame, from fear? Can we even separate these emotions out? I pose these questions to illustrate how this diagnostic question raises more questions than it answers, once again potentially limiting the manual's prized goal of clinical utility.

To determine if the trauma has inhibited one's desire to socialize and to participate in her $\leftrightarrow$ his usual activities, clinicians ask: "Are you much less interested in activities in which you used to participate?" (Nussbaum 91). Like previous diagnostic questions, this one could also be on the list for diagnosing many other mental disorders besides traumatic ones. Nowhere is there an inquiry into how the traumatic event(s) may have led to decreased interest in one's usual routine. More importantly, the question assumes that if a patient answers "yes," it may mean she $\leftrightarrow$ he is negative when, in fact, a patient may be less interested in certain activities because they put her $\leftrightarrow$ him at risk of further trauma. For example, if an athlete seriously injured during a football game loses interest in football, it may not indicate PTSD, but that he is taking appropriate cautions to avoid reinjuring himself. Furthermore, trauma may more generally change the kinds of activities a survivor enjoys. This change could be a healthy adaptation, not a sign of negativity.

To determine if the trauma has impacted one's social relationships, clinicians ask: "Do you feel detached or estranged from the people in your life because of this experience?" (Nussbaum 91). This question, like the preceding one, assumes that change indicates negativity. In fact, a trauma survivor may feel detached or estranged because the people in her $\leftrightarrow$ his life are not supportive. For example, if a survivor of sexual assault at the hand of a priest disconnects from his parents, who staunchly support the Catholic 
Church, that disconnect may not indicate PTSD, but that he is protecting himself from further abuse. If a patient does feel detached or estranged from others when those others are supportive, the phrasing of this question presumes that she $\leftrightarrow$ he can link negative relationship changes with the trauma, when the patient may not know why she $\leftrightarrow$ he feels estranged.

To determine if the trauma has limited one's ability to experience positive emotions, clinicians ask: "Do you find that you cannot feel happy, loved, or satisfied? Do you feel numb, or like you cannot love?" (Nussbaum 91). Like previous diagnostic questions, this one could also be on the list for diagnosing many other mental disorders besides traumatic ones. Nowhere is there an inquiry into how the traumatic event(s) may have limited one's ability to experience positive emotions. More importantly, like the question about negative emotions, this question lumps a range of so-called "positive" emotions - happiness, love, satisfaction — into one thought, which may lead to faulty answers. If a survivor does not experience one of these emotions but does experience others, she may answer in the negative. If a survivor does not feel one of these emotions but does experience others, and answers the question in the affirmative, does his answer indicate PTSD or some other ailment, such as a sexual dysfunction, substance abuse issue, or personality disorder - all of which can interfere with positivity? If a former domestic violence survivor does not feel loved by her current (non-abusive) husband, does that mean that she may have PTSD, or that she is working through trust issues induced by her previous abusive marriage? How does $D S M-5$ discern happiness, from love, from satisfaction? Can we even separate these emotions out? Again, I pose these 
questions to illustrate how this diagnostic question raises more questions than it answers, potentially limiting the manual's prized goal of clinical utility.

\section{Traumatic Arousal}

After determining that a patient has been exposed to (a) traumatic event(s), that she $\leftrightarrow$ he continues to reexperience and actively avoids reminders of the event(s), and that she $\leftrightarrow$ he has experienced negative changes mentally and emotionally, clinicians then examine how trauma may impact the patient's degrees of arousal and reactivity. Labeled

"Criterion E," this symptom set refers to traumatic arousal. Symptoms of traumatic arousal are reckless and/or destructive behaviors, which can include irritability and anger, self-destructiveness, hypervigilance, "exaggerated startle," concentration difficulties, and/or sleep difficulties (DSM-5 272). Traumatic arousal is a cognitive-emotional agitation that can produce disruptive or aggressive behaviors towards oneself and/or others.

By itself, the noun "arousal" has multiple meanings that can extend DSM-5's conception of arousal, and pertains to acts that stir, excite, or awaken - the opposite of the depressing emotions and thoughts described in Criterion D, traumatic negativity ("Arousal"). Combining the DSM-5 and Oxford English Dictionary definitions of arousal can help us understand trauma as a metaphorical alarm clock, without a snooze button, going off in the brain. We can understand traumatic arousal as an anxious awakening that produces excess - excess anger, anxiety, or excitement, directed inward or outward - that interferes with inner peace, concentration, and sleep. Trauma can both depress, as described in the previous section on traumatic negativity, and awaken to a 
startling degree, setting in motion what traumatologist Judith Herman calls, the central dialectic of trauma - "[t]he conflict between the will to deny horrible events and the will to proclaim them aloud" (1). The urge to deny trauma may signal traumatic avoidance, whereas the urge to proclaim trauma as loudly and as often as possible may signal a kind of compulsive reexperiencing, traumatic intrusion. Contextualized here within DSM-5's concept of traumatic arousal, Herman's dialectic can be explained as a primarily internal conflict that emerges from traumatic experience—one that can induce a depressed state, on the one hand, and an anxious one, on the other. Thus, traumatic negativity and traumatic arousal symptoms may actually intersect more with the preceding symptoms than $D S M-5$ 's classification of them (as separate symptom clusters) suggests.

To assess patients' experiences of traumatic arousal symptoms, clinicians inquire into irritability or aggressiveness, recklessness, hypervigilance, exaggerated startle, impaired concentration, and sleep disturbance: "Do you often act very grumpy or get aggressive?... Do you often act reckless or self-destructive?... Are you always on edge or keyed up?... Do you startle easily?... Do you often have difficulty falling asleep or staying asleep, or do you often wake up without feeling rested?" (Nussbaum 91). In order to be diagnosed with PTSD, a patient must experience at least two of these behaviors for at least one month after the event has passed. None of these questions ask directly about the traumatic event(s), which suggests that formulating a PTSD diagnosis based on a patient's answers to them is contingent upon her $\leftrightarrow$ his ability to link arousal behaviors with trauma. In addition, all of these questions direct patients to give their feelings particular labels (e.g., to identify hypervigilance as feeling "on edge or keyed up") and moreover, to link those feelings with particular actions (e.g., irritability is measured by 
"act[ing] very grumpy"). In point of fact, survivors may have other language to describe arousing feelings, and/or they may not necessarily link arousing feelings to reactive behaviors. Arousing feelings and reactive behaviors may also stem from non-traumatic stimuli. Taken together, these discrepancies suggest that the recommended ways for clinicians to assess traumatic arousal may put words in survivors' mouths, reframing the traumatic event(s) in ways that distort survivors' actual emotional and behavioral experiences, and may even lead to misdiagnosis.

\section{Effects of Trauma Symptoms}

In trying to make a PTSD diagnosis, clinicians rely primarily upon the above five DSM-5 symptom clusters: traumatic exposure, traumatic intrusion, traumatic avoidance, traumatic negativity and traumatic arousal (Criterion A-E). The next step in the diagnostic process, Criterion $\mathrm{F}$, involves assessing the duration of those symptoms which, as discussed in the preceding analyses of each symptom cluster, must be one month or more following the traumatic event(s). After assessing the presence and duration of symptoms, clinicians then assess the effects of those symptoms, Criterion G, or how symptoms may cause "impairment in social, occupational, or other important areas of functioning" (DSM-5 272). A person exposed to trauma may experience some intrusion, avoidance, negativity, and/or arousal symptoms in the aftermath of trauma, but these experiences alone do not necessarily indicate that a patient has PTSD. The intensity of those experiences - in particular, the extent to which symptoms interfere with one's usual day-to-day functioning - ultimately determine the presence of absence of this disorder. 
For example, a transgender rape survivor may experience nightmares and guilt or shame about the rape, questioning whether or not it even happened or if they caused it by transgressing gendered norms, roles, and stereotypes. They may even experience some difficulty returning to work or practicing self-care after the rape. However, those trauma symptoms may not interfere much, if at all, with their ability to hold down a job, to relate with other people, or to feed, house, and clothe them. Thus, a clinician may not diagnose them with PTSD. They may not even seek out therapy in the first place. However, if another rape survivor experiences those same trauma symptoms and begins to arrive late to or miss days of work altogether, to increasingly struggle to hold a conversation, and to eat regularly and nutritiously, a clinician would be far more likely to diagnose that person with PTSD. Functionality demarcates who is traumatized from who is not, who is pathological and who is normal.

Both in its description of PTSD and in its definition of mental disorder, DSM-5 suggests that a PTSD diagnosis relies ultimately upon a particular idea about normalcy: functionality. According to DSM-5's standards for determining mental illness-through "social, occupational, or other important areas of functioning"- to function is to work, rendering productivity among the most important criterion for leading a normal life. To function is also to meet one's own basic needs, rendering independence yet another important criterion for leading a normal life. In addition, to function is also to interact regularly and to even make nice with others, rendering sociability yet another important criterion for leading a normal life. DSM-5 normalizes functionality, pathologizing alleged dysfunctionality: unemployment, a need for outside help in meeting basic needs, and/or an antisociality. $D S M-5$ renders dysfunctionality a failure, and a personal failure at that. 
Dysfunctionality, as conceptualized by $D S M-5$, does not necessarily or universally indicate problems. For some, it may indicate a personal preference for solitude, for example. It may also indicate a different cultural perception of "career" as a means to an end rather than an end-goal in and of itself. The diversity of ways that individuals, communities, societies, and cultures conceive of functionality suggests that $D S M-5$ 's yardstick for measuring psychological health (normality) versus illness (pathology) may not produce the "common language" or "consistent and reliable diagnoses" the manual purports to establish.

\section{Substances and Other Medical Conditions}

The final step in making a PTSD diagnosis is determining whether substances or preexisting medical conditions may have produced intrusion, avoidance, negativity, and/or arousal symptoms. As Criterion H states, a clinician can only diagnose a patient with PTSD if "[t]he disturbance is not attributable to the physiological effects of a substance (e.g., medication, alcohol) or another medical condition" (DSM-5 272). Clinicians may encounter great difficulty satisfying this final requirement, since trauma often coincides with other medical conditions or with substance abuse, a common form of self-medicating in the face of overwhelming suffering.

For example, the National Child Traumatic Stress Network, a non-profit organization that advocates for children and adolescents exposed to traumatic events, states that more than half of young people diagnosed with PTSD, up to $59 \%$, go on to abuse substances (Cook \& Polgar 1). For someone already using drugs or alcohol, PTSD may make sobriety even more difficult as "exposure to reminders of the traumatic event 
have been shown to increase drug cravings" (Cook \& Polgar 2). Substance abuse may increase the likeliness that any person, regardless of age, will develop PTSD as substance abuse often goes hand in hand with risky situations that can easily become traumatic (e.g., violence, death). Furthermore, people use recreational drugs to alleviate nightmares or to suppress aggression. As the symptom cluster of traumatic avoidance (Criterion C) suggests, a hallmark of PTSD is a desire to forget. Both pharmaceutical and recreational drugs, which one does not necessarily need medical insurance to access, provide a ready means of escaping trauma - albeit a temporary one. For these reasons, $D S M-5$ 's final stipulation that symptoms not coincide with effects of substances may prove inappropriate in trying to make or eliminate a PTSD diagnosis. Trauma and substance abuse are too deeply intertwined to distinguish from one another.

The other stipulation mentioned in Criterion $\mathrm{H}$, that symptoms not coincide with other medical conditions, may also prove inappropriate. Traumatic events may induce other medical conditions, such as intestinal problems, chronic pain, or heart failure. Some medical conditions can even induce trauma, particularly if those conditions are fatal. In attempting to isolate mind from body, trauma from other medical conditions, $D S M-5$ may lead clinicians to overlook trauma as a possible effect or a possible cause of those conditions. It may also lead clinicians to overlook the ways in which the body records trauma, sometimes in far more tangible ways than a talk-based diagnostic interview or therapy session does. Trauma plays out through the body and by definition, often occurs directly to the body, potentially rendering traumatized bodies more vulnerable to other medical issues. For these reasons, $D S M-5$ 's insistence that clinicians isolate trauma from other medical conditions may prove impossible in trying to make or eliminate a PTSD 
diagnosis. These exclusions regarding substances and other medical conditions pay lip service to the many factors that come into play with making a diagnosis, but are not at all practical for actual trauma survivors who may be suffering the most.

\section{Conclusion: Diagnosing Events}

DSM-5's classification of PTSD constructs trauma as a failure to fight off an event. Perhaps a more effective medical intervention into trauma would be to shift the locus of responsibility for presenting certain symptoms, in order to be diagnosed with and thereby receive treatment for PTSD, from individual patients towards systems, institutions, ideologies, and practices that enable traumatic events in the first place. In essence, we could shift diagnosis away from patients and towards traumatic events-a reversal of modern medicine's move toward pathologizing bodies rather than diseases. Empirical evidence is often rare in the case of rape trauma, making it difficult to theorize about people's experiences of rape.

It is, however, possible to theorize about the event of rape, about what rape is. Rather than asking, How does this rape survivor manifest intrusion, avoidance, negativity, and arousal symptoms?, we might ask, How is rape itself an act of traumatic intrusion, traumatic avoidance, traumatic negativity, traumatic arousal? I explore these questions in the subsequent chapters of this dissertation by examining how different literary and cinematic texts communicate with and challenge DSM-5's discursive construction of trauma through PTSD. Rhetorician Lucille McCarthy, Professor of English at the University of Maryland Baltimore County, and psychiatrist Joan Page Gerring, Research Scientist and Medical Director at Johns Hopkins Medical Center, 
analyze the revision process from $D S M-I I I$ to $D S M-I V$. As they conclude in "Revising Psychiatry's Charter Document: DSM-IV," the revision process as well as the manual itself are deeply rhetorical, deeply ideological, challenging assumptions of objectivity in Western medicine. They write,

Our overall conclusion... is that the revision of the DSM manual functions less to change the text than to achieve the following three effects: (a) to further solidify the dominance of the biomedical model of mental disorder within psychiatry, (b) to maintain the position of psychiatry as the highstatus profession among competing disciplines within the mental health field, and (c) to achieve acceptance of psychiatry as a mature, researchbased specialty within medicine. (149)

Based on my analysis of how DSM-5 constructs PTSD, I posit that we extend their findings one step further, to trauma survivors themselves. As the $D S M$ shores up the dominant biomedicalization of trauma, among other so-called medical conditions, reinscribes psychiatry as "the high-status profession" within mental healthcare, and medicalizes psychiatry as it objectifies individual patients as "members of populations grouped according to observable symptoms" (150). This kind of objectification erases sociocultural variations in how people experience and respond to trauma. It psychologizes trauma, obscuring emotional, bodily, spiritual, and other manifestations of trauma symptoms and therapeutic interventions. It renders obsolete, in the diagnostic process, the testimonies of those who have lived through traumatic events.

As my analysis of $D S M-5$ in this chapter suggests, trauma has become heavily psychologized, heavily medicalized. The manual's discursive construction of PTSD is 
fraught with Western ideologies and contradictions, underscoring a need to venture outside of clinical settings to assess the efficacy of PTSD symptoms and diagnostic criteria. For survivors of sexualized violence in particular, whose bodies are already marked with shame and blame, untangling DSM-5's classification of PTSD becomes especially critical. To that end, the chapters that follow function as case studies of several contemporary works of literature and film that contend with rape in global settings. The next chapter, a case study of Stieg Larsson's Girl with the Dragon Tattoo and Niels Arden Oplev's and David Fincher's film adaptations, analyzes how these texts discursively construct trauma as a catalyst for feminist vigilantism. The construction of trauma in Girl with the Dragon Tattoo, I argue, challenges DSM-5's construction of trauma survivors as victims of circumstance (traumatic exposure) who reexperience traumatic events against their will (traumatic intrusion) and who actively avoid associations with the event (traumatic avoidance). While protagonist Lisbeth Salander is characterized as a woman disconnected from herself and others (traumatic negativity), and who engages in aggressive behavior (traumatic arousal), her disconnection and aggression are attributed not to personal dysfunctions, but to systemic, institutional failures. Thus, I also argue that Girl with the Dragon Tattoo moves us closer to an understanding trauma as a social and not an individual phenomenon. 


\section{CHAPTER III}

\section{A CASE STUDY OF LITERARY AND CINEMATIC TRAUMA DISCOURSES: DISCURSIVE FORMATIONS OF TRAUMA IN}

\section{GIRL WITH THE DRAGON TATTOO}

In the previous chapter, I demonstrated how trauma has become heavily psychologized through The Diagnostic and Statistical Manual of Mental Disorders V (DSM-5) and its epistemo-politics of health and wellness; the discursive construction of trauma as an individual pathology_one that arises from "dysfunction in the psychological, biological, or developmental processes underlying mental functioning," and that constitutes a personal failure to assimilate catastrophic events - silences, erases, and otherwise renders invisible the diversity of ways in which humans experience and respond to catastrophe (DSM-5 20). To assess the efficacy of such assumptions and ideologies about trauma naturalized by $D S M-5$ and its contexts, this chapter conducts a case study of sexualized trauma in Stieg Larsson's posthumously published novel, and Niels Arden Oplev's and David Fincher's film adaptations of, Girl With the Dragon Tattoo. Reading representations of rape in Girl With the Dragon Tattoo alongside the American Psychiatric Association's (APA's) symptomatology of posttraumatic stress disorder (PTSD) exposes incongruities between embodied experiences and the biomedicalization of trauma. 
A scathing critique of white misogyny and neoliberalism in turn of the twentyfirst century Sweden, Girl With the Dragon Tattoo constructs trauma as an institutional rather than an individual failure that, in fact, perpetrates the very violence that begat the trauma. The novel and films thereby elicit distrust and animosity towards Sweden's social services - especially its police, legal guardianship, and mental healthcare systems - in addition to its wealthy industrial corporate structures. Sexualized trauma in Dragon Tattoo stems from a failure of public institutions to care for the publics they allegedly serve, and from a white misogynist backlash against women's increasing social and sexual mobilities in Sweden.

Moreover, Dragon Tattoo constructs sexualized trauma as a vehicle for eradicating male coercive control, a catalyst for feminist vigilantism. Individual characters' violent responses to sexualized violence are embedded in a larger feminist project of dismantling misogyny. Murder enables Harriet Vanger, the largely-unseen centerpiece of Dragon Tattoo's story, to end her father's incestual abuse, and revenge rape enables titular antiheroine Lisbeth Salander to set the terms of her release from her sadistic legal guardian, Advokat Nils Bjürman, and from her social status as paranoid schizophrenic and legally incompetent. That said, privileges afforded to Harriet Vanger and Lisbeth Salander, such as technical acumen, a photographic memory, familial wealth and social connections, and lucrative employment, suggest that Dragon Tattoo's brand of feminist vigilantism is a privileged feminism. Lisbeth is a highly-skilled, well-paid computer hacker who can recall visual information after only brief exposure. Harriet has the privilege of mobility afforded by her family's prosperous business, the Vanger Corporation, even though she escapes the family home when she is sixteen. While the 
agency that feminist vigilantism affords is confined to white, middle- to upper-class women, Harriet and Lisbeth, their vigilantism catalyzes justice for themselves, as well as for Jewish women across Sweden who are sexually violated and murdered by white men. As this chapter posits, Girl With the Dragon Tattoo conditions us to perceive trauma differently: as a systemic pathology that catalyzes feminist vigilantism, thereby challenging $D S M-5$ and the medical authority it garners, and denaturalizing the (bio)medicalization of "trauma victim" as a frightened, helpless, dependent object of pity. Trauma, then, sets in motion a retributive cycle of violence produced by racialized misogyny. To demonstrate how Girl with the Dragon Tattoo conditions us to perceive trauma as a systemic pathology that catalyzes feminist vigilantism, this chapter explicates how the novel and film adaptations portray sexualized trauma as a product of white misogyny, authorize violence as a just response to sexualized violence perpetrated by men against women, and ultimately challenge the (bio)medicalization of trauma.

\section{Trauma: A Systemic Pathology}

Girl With the Dragon Tattoo portrays trauma as arising from sexualized violence perpetrated by men against women — what traumatologist Judith Herman, M.D. describes as, "a method of political control, enforcing the subordination of women through terror" (30). Dragon Tattoo casts the two principal heroines, Lisbeth Salander and Harriet Vanger, as underestimated, misunderstood survivors of coercive male authority in the Swedish Guardianship Agency, Swedish Secret Police, and St. Stefan’s Psychiatric Hospital (Lisbeth), and the prestigious Vanger Corporation (Harriet), characterizing their traumas as products of institutionalized misogyny. Additionally, Dragon Tattoo casts 
men in positions of authority who violate Lisbeth and Harriet as narcissistic, sadistic predators of women (i.e., vulnerable bodies) who intend to "convince [women] that [they, the perpetrators, are] omnipotent, that resistance is futile, and that [women's] li[ves] depend upon winning [perpetrators'] indulgence through absolute compliance" (Herman 77). Gottfried and Martin Vanger terrorize Harriet Vanger, their daughter and sister, respectively, with rape. Advokat Bjürman rapes Lisbeth on two separate occasions when Lisbeth requests access to her financial accounts. Lisbeth's father, Alexander Zalachenko (a.k.a., "Zala"), a member of the counterespionage Swedish Secret Police, physically and sexually abuses Lisbeth's mother, Agneta Sjölander, in front of Lisbeth. Additionally, prominent, yet perverse psychiatrist, Dr. Peter Teleborian, institutionalizes, restrains, and medicates Lisbeth against her will after she tries to stop Zala once and for all by setting him on fire.

Lisbeth has zero social status. A strict form of social welfare, guardianship laws in Sweden mandate that as Lisbeth's guardian, Advokat Nils Bjürman "shall take over all of the client's legal powers," including management of her finances (Larsson 225). When Lisbeth shows up at Bjürman's office to request access to her bank account so that she can replace her laptop, destroyed during a mugging at the subway station, Bjürman agrees to cut her a check if she gives him a blowjob. " 'If you're nice to me, I'll be nice to you," " Bjürman taunts as he pulls Lisbeth's head to his crotch (222). Lisbeth immediately recognizes this act as "a display of strength by an enemy force-an indication that [...] she was at the mercy of [Bjürman's] discretion and defenceless" (221). After Bjürman completes the oral rape, Lisbeth goes directly home from Bjürman's office, showers, eats "two sandwiches with cheese and pickles," and sits down 
to strategize her revenge (227). With no legal recourse to incriminate Bjürman on her own, Lisbeth devises her own "justice."

Lisbeth's immediate revenge response to Bjürman's oral rape bypasses altogether the shame, doubt, and psychological captivity that Judith Herman, M.D. theorizes as innate responses to "the violation of bodily integrity" intrinsic to rape (53). As Herman explains, rape renders women unable to "maintain [their] own separate point of view while remaining in connection with others" (53), provoking an "existential crisis" in which survivors, not perpetrators, feel guilty (51). We expect Lisbeth to shame and doubt herself, not to methodically shower, eat, and then strategize for revenge just moments after scrubbing Bjürman's ejaculate out of her mouth with soap.

Lisbeth subverts our expectations because she is vulnerable - not the shared human condition of vulnerability that connects us, but what feminist philosopher Debra B. Bergoffen describes as the "historically contingent political distribution" of vulnerability that renders women's bodies vulnerable to rape and other forms of sexualized violence perpetrated by men (102). Further, Lisbeth is made vulnerable because she subverts social norms. Lisbeth belongs to a population Herman says is "most at risk" of both experiencing rape, and of consequentially, developing psychological trauma (69). Lisbeth is female, an alleged paranoid schizophrenic and legal incompetent, "disempowered" and "disconnected from others" (60). Moreover, Lisbeth behaves "as though she were free $[\ldots]$ not observing conventional restrictions on dress, physical mobility, and social initiative" (69). Lisbeth embodies a refusal of gendered norms. In point of fact, our introduction to Lisbeth in the novel is filtered through an authoritative male gaze, that of Dragan Armansky, the CEO and COO of Milton Security 
and Lisbeth's boss, who characterizes Lisbeth by her physical appearance: "hair as short as a fuse, and a pierced nose and eyebrows [...] a wasp tattoo about an inch long on her neck, a tattooed loop around the biceps of her left arm and another around her left ankle [...] a dragon tattoo on her left shoulder blade" (Larsson 38). As Armansky's description illustrates, Lisbeth is perceived, first and foremost, as breaking with gendered conventions. The male gaze sexualizes Lisbeth, by way of her non-normative female body — short hair, visible tattoos, piercings—as is evidenced by Armansky's conclusion that Lisbeth appears "as though she had just emerged from a week-long orgy with a gang of hard rockers" (ibid). Moreover, the male gaze infantilizes Lisbeth—her small stature, "slender bones" that "ma[ke] her look girlish and fine-limbed with small hands, narrow wrists, and childlike breasts. She was twenty-four, but she sometimes looked fourteen" (ibid). Others', especially men's, preoccupation with Lisbeth's unconventional female body dramatizes the impenetrability she displays when she enacts revenge against her father (setting Zala on fire), her legal guardian (revenge raping Bjürman), and finally, the man responsible for Harriet's disappearance, Martin Vanger (who Lisbeth chases off the road to his death). Her revenges are displays of invulnerability to internalizing the terror of sexualized trauma, and of rejecting the male gaze, even as Lisbeth's unconventionality renders her vulnerable and "at risk."

Justice demands a revealing of our innate vulnerability, Dragon Tattoo advocates - a willingness to encounter pain, injury, trauma. Lisbeth's revenges bring individual perpetrators to justice (Zala, Bjürman, Martin), but they also expose the sadistic, racist misogyny of Sweden's legal system. For example, Lisbeth's revenge against Bjürman necessitates extra-state violence, operating outside of the law because 
"police [are] not an option," pointing up how law enforcement (among other social institutions designed to uphold the "freedoms" of their citizenry) does not fulfill its intended function (Larsson 227). Given Lisbeth's social status as a ward of the state, she concludes that the police would not believe her if she filed a report against Bjürman for raping her.

Moreover, Lisbeth has come to recognize the police as "a hostile force who over the years ha[s] put her under arrest or humiliated her" (Larsson 227). Social institutions and the white misogynists controlling them have utterly betrayed Lisbeth. Lisbeth understands that she is "legal prey" (228). Lisbeth understands Bjürman's rapes as efforts to keep her dependent, silent, penetrable. Rape is institutionalized captivity. Relying on psychiatric and criminal records that pathologize Lisbeth "as cunning and unjustifiably violent," Bjürman assumes Lisbeth does not have the competence to manage her own financial accounts, to recognize his sadism as such, or to stave off his sexual advances (ibid). Bjürman underestimates Lisbeth, calling her "fucking retarded," believing that he can get away with abusing her (223). Putting an end to Bjürman's perpetrations against Lisbeth (and potentially, many other women as well) requires vulnerability—that is, transforming sexualized injustices requires that the very systems through which such injustices are perpetrated confront their own brokenness, a task that Lisbeth's feminist vigilantism sets in motion.

Contrary to Bjürman's assumptions about Lisbeth, she is, in fact, exceedingly competent; the institutionalized (normalized) perception of her as psychotic and delinquent—as powerless and therefore, susceptible and abusable—denies her opportunities to exercise that competency openly. For example, when Lisbeth is 
institutionalized after setting Zala on fire, and she tells Dr. Teleborian about Zala's physical and sexual abuse of her mother, Agneta, Dr. Teleborian falsely diagnoses Lisbeth as paranoid schizophrenic, which results in her social status as legally incompetent, which puts her under court-mandated supervision of a legal guardian into adulthood, when we meet her in her early twenties in Girl with the Dragon Tattoo. Enlisted by the Swedish Security Police to protect Zala, Dr. Teleborian straps Lisbeth down to force-feed her psychotropic medications. During her two years at St. Stefan's Psychiatric Hospital, Lisbeth spends more than half of that time (381 days) in restraints. Using Dr. Teleborian's falsified psychiatric diagnosis as justification, the Law of Sweden declares Lisbeth "incompetent"-a label that ultimately puts her into Bjürman's "care." While Zala sustains severe burns_- his scarred body a testament to Lisbeth's refusal to comply with male abuses of power, and to her insistence upon retribution-Zala survives. He also evades any legal repercussions for abusing Agneta, due to his prestigious position with the Swedish Security Police.

As Lisbeth's history of institutionalization suggests, male violence against women catalyzes and structures Girl With the Dragon Tattoo. It functions as the inciting event that sets the entire Millennium Trilogy in motion, and it links characters across space and time, representing sexualized trauma on its systemic scale, conditioning us to perceive violence against women as the actant tying different storylines together. Dragon Tattoo's inciting incident, the conviction of investigative journalist Mikael Blomkvist for libeling corrupt financier Hans-Erik Wennerström, sets in motion a series of events related to solving the rapes and murders of multiple women in Sweden. Dragon Tattoo centers on the unsolved case of Harriet Vanger, a sixteen year-old girl who mysteriously 
disappeared from her family's home on Hedeby Island, a fictional place in eastern Sweden, in 1966. Harriet's great-uncle Henrik Vanger, retired CEO of the Vanger Corporation, believes that someone in the misogynistic, neo-Nazi family business, fearing that Harriet (a woman) might assume leadership of the family business, murdered Harriet and disposed of her body. Henrik hires Mikael, who later hires Lisbeth Salander as a research assistant, to get to the bottom of Harriet's disappearance.

As the plot unfolds, Mikael and Lisbeth link Harriet's alleged murder to the rapings and killings of at least five Jewish women across Sweden, ultimately discovering that Harriet is still alive. When they locate Harriet in Australia, she discloses that incest and child sexual abuse at the hands of her father, Gottfried Vanger and brother, Martin Vanger drove Harriet to escape: “ 'I was all alone [...] my father was also an insane killer of women [...] My father molested me for a year [...] My father could have raped me in the middle of the living room right before [my mother's] eyes and she wouldn't have noticed [...] I couldn't bring myself to say anything. The whole world would have found out' " (Larsson 493-94). As Harriet's testimony here suggests, she, like Lisbeth, had minimal social support for bringing her male perpetrators to justice. While Harriet is not vulnerable in the same ways as Lisbeth, insofar as Harriet's violence against her father leads to her escape (a conscious decision) rather than to institutionalization (a form of coercive control), Harriet's own mother, Isabella Vanger, willfully ignored Gottfried's abuse. Moreover, given the widespread prestige of the Vanger Corporation, Harriet knew that if she told anyone her story, it would either become public and destroy both business and family, or it would discredit, ostracize, and likely subject Harriet to even more brutal violence. Sanctioned by familial and corporate structures, sexualized violence shrinks 
Harriet's world while it also lays the foundation for exposing the corruption of the Vanger Corporation. Such vulnerability puts an end to Gottfried's and Martin's perpetrations against Harriet (and many other women) — that is, transforming sexualized injustices requires that the very systems through which such injustices are perpetrated confront their own brokenness, a task that Harriet's and later, Lisbeth's feminist vigilantism enacts.

While sexualized violence terrorizes Harriet, exposing the Vanger Corporation for its racist, misogynist brutality by way of solving Harriet's case facilitates justice for other women victims of male rape. As Harriet's testimony unfolds, we learn that Harriet's desperation leads her to take justice into her own hands, a choice that will eventually liberate her and other women. Harriet's testimony continues:

"On August 7, 1965, my father forced me to go out to his cabin [...] My father was drinking, and he tried to forced himself on me. But he couldn't get it up and he flew into a drunken rage. He was always... rough and violent towards me when we were alone, but this time he crossed the line. He urinated on me. Then he started telling me what he was going to do to me. That night he told me about the women he had killed. He was bragging about it. He quoted from the Bible [...] He put a T-shirt around my neck and pulled it as tight as he could $[\ldots]$ and for the first time that night he managed to complete the rape $[. .$.$] But he was so drunk that$ somehow I managed to get away $[. .$.$] He came staggering after me [\ldots]$ I was strong enough to shove an old drunk into the water. I used an oar to hold him under until he wasn't struggling any more [...] And when I 
looked up, there stood Martin. He looked terrified, but at the same time he was grinning $[\ldots .$.$] From that moment I was at his mercy. He came up to$ me, grabbed me by the hair, and led me back to the cabin — to Gottfried's bed. He tied me up and raped me while our father was still floating in the water $[\ldots]$ From that day on, I was in his power. I did what he told me to do [...] [My mother] decided that Martin needed a change of scenery after his father's tragic death. So she sent him to Uppsala. Of course this was because she knew what he was doing to me, and it was her way of solving the problem [...] I was told that he wouldn't be coming to [the annual] family gathering, that he was staying in Uppsala. But obviously he changed his mind, and suddenly there he was on the other side of the street, staring at me. He smiled at me. It felt like a hideous dream. I had murdered my father, and I realised that I would never be free of my brother. Up until then, I had thought about killing myself. I chose instead to flee.” (Larsson 495-97)

As Harriet's testimony illustrates, violence against women sets Dragon Tattoo in motion. Gottfried's sadistic hatred of women provokes feminist vigilantism, in the form of murder, which intensifies Martin's sadistic misogyny, which intensifies Isabella's denial of her son's violence, which prompts Harriet's escape, which becomes the centerpiece of Dragon Tattoo's story.

A mystery for forty years, Harriet's disappearance leads the aging Henrik to recruit Mikael Blomkvist to investigate the case one last time before Henrik passes away. Mikael's investigation leads him to Harriet's diary, which contains a list of seemingly 
random female names and numbers that Lisbeth and Mikael's daughter, Pernilla Blomkvist, decode as Bible passages from the book of Leviticus about proper punishments for women's sins. These passages help Mikael and Lisbeth discover that under the cover of Vanger Corporation business trips, Martin and Gottfried rape and murder Jewish women across Sweden in the manner these Bible verses describe.

Ultimately, this insight leads Mikael and Lisbeth to capture Martin and to figure out that Harriet was not murdered, but rather, that she escaped Sweden altogether. Girl with the Dragon Tattoo thereby constructs male violence against women as both interpersonalbetween Harriet, Gottfried, Martin, and the murdered Jewish women — and institutional— at once sanctioned and concealed by the corporate authority of the Vanger Corporation. In catalyzing and structuring Girl with the Dragon Tattoo, violence against women is configured as both an interpersonal trauma and an infectious systemic pathology.

In addition to the domino effects of Harriet's victimization, Dragon Tattoo's indictment of actual violence against women in Sweden also calls attention to the systematicity of rape. Larsson uses factual statistics on gendered violence to introduce each of the novel's four parts. Originally titled Men Who Hate Women, ${ }^{19}$ the title of Oplev's Swedish film adaptation, Girl With the Dragon Tattoo opens with, "Eighteen percent of the women in Sweden have at one time been threatened by a man" (Larsson 9). The coupling of this statistic with Part One's title, "Incentive" suggests that the "threat" of male violence provides "incentive" for Lisbeth and Mikael to find and uproot the source of that violence. As this first section reveals, the "threat" of Mikael's left-wing

\footnotetext{
${ }^{19}$ In the Swedish film, the title only appears in English as "Girl With the Dragon Tattoo"_-perhaps a more palatable title for global audiences. Girl With the Dragon Tattoo title does not implicate readers/viewers in the same way that Men Who Hate Women does. Rather, the Dragon Tattoo title suggests that the story centers on an individual character rather than on normalized social prejudices and violence.
} 
Millennium Magazine going under, after he is charged with libel against Wennerström, provides Mikael the incentive to take leave from Millennium Magazine and work for Henrik Vanger on the Harriet Vanger case, setting Dragon Tattoo's central narrative in motion.

Transitioning from threatened to actualized violence against women, the second part of the novel opens with the statistic, "Forty-six percent of the women in Sweden have been subjected to violence by a man" (Larsson 127). While the number of women who have been threatened with violence is under $20 \%(18 \%$, as the first statistic states), this second statistic illustrates that at least half of Sweden's female population has experienced worse: actualized violence. Part Two, entitled "Consequence Analyses" opens with Blomkvist traveling to Hedestad to begin working for Henrik Vanger, and closes with Henrik's proposal to save Millennium Magazine from bankruptcy by becoming a part owner. The "consequence" of Mikael trying to expose Wennerström is Mikael having to work for another corporate giant, the Vanger Corporation, which leads Mikael to "analyze the consequences" of his career-long commitment to indicting corporation corruption. Also during Part Two, Lisbeth Salander endures both of Advokat Bjürman's rapes, and she enacts her revenge by working outside of the system—a stark contrast to Blomkvist, who winds up accepting Henrik's offer to buy a share in Millennium Magazine and to continue working for the Vanger family. The placement of this statistic on actualized violence against women in Part Two of the novel suggests that we consider the catastrophic effects to Sweden as a whole when almost half of the country's female population experiences actualized male violence. 
Part Three, "Mergers," opens with a statistic on violence against women outside of sexual relationships, a contrast to frequently-spotlighted dating violence or intimate partner violence: "Thirteen percent of the women in Sweden have been subjected to aggravated sexual assault outside of a sexual relationship" (Larsson 273). Given its placement just after Part Two, in which both of Bjürman's rapes of Lisbeth and Lisbeth's revenge rape of Bjürman occur, this statistic describes the experiences of women sexually assaulted by men with whom they did not have sexual relationships: Lisbeth, Harriet, and the Jewish women her father and brother murdered. "Mergers" opens with Mikael completing his prison sentence, the result of being charged with libeling Wennerström (which, as the analysis of Part Two's statistic shows, functions as the incentive for Mikael to work for Henrik Vanger), and closes with Martin Vanger imprisoning Mikael in his basement, just as Mikael and Lisbeth are uncovering Martin's true identity as a killer of women. The result of merging Millennium Magazine and the Vanger Corporation puts Mikael in life-threatening danger, at the same time as it facilitates the resolution of Harriet's and other women's victimization and ultimately, the clearing of Mikael's name for libeling Wennerström.

Part Four, "Hostile Takeover," comments on the inefficacy of legal institutions to intervene in violence against women, opening with the statistic that "Ninety-two percent of women in Sweden who have been subjected to sexual assault have not reported the most recent violent incident to the police" (Larsson 445). In this final part of the story, Lisbeth chases after Martin Vanger on her motorcycle, driving him off the road to his death. Lisbeth and Mikael locate Harriet in Australia, bringing her back to Sweden to reunite with her uncle, Henrik Vanger. Additionally, Lisbeth stops Wennerström from 
committing further financial fraud by way of emptying all of his financial accounts and seeing to it that Wennerström is killed. The coupling of the "Hostile Takeover" section title and the statistic about Sweden's legal system failing women suggests that change must come about through systemic overhaul—a project that requires vulnerability, remaining open to destroying and/or reinventing broken systems. The Judiciary of Sweden is ineffective at best, and corrupt at worst. When only $8 \%$ of women victims report sexual assault to police, women are not safe—especially when those same victims have reported assaults in the past. According to the Rape, Abuse and Incest National Network (RAINN)—a U.S.-based group that partnered with Dragon Tattoo's film distributor, Music Box Films, to bring Oplev's film to over 125 college campuses and rape crisis centers across the U.S., screening it for free and facilitating discussion groups about sexualized violence-victims who do report rape are often subjected to questions and accusations normalized by rape myths that women are overly-sensitive reactionaries, codependents, and relationship addicts; and that rape is an enactment of women's deepseated rape fantasies.

Girl with the Dragon Tattoo's original Men Who Hate Women title, coupled with its strategic placement of statistics about violence against women in Sweden, suggests parallels between fictionalized and actualized violence in a nation notorious for its relative gender equity compared to the rest of the globe. ${ }^{20}$ Statistics frame and link the events within each section of the novel, bookending Harriet's and Lisbeth's histories with factual material. Statistics prompt us to read Lisbeth's and Harriet's histories as representations of actual biographies, ultimately configuring Dragon Tattoo as an

\footnotetext{
${ }^{20}$ For more on Sweden's global reputation as the most gender-equal nation in the world, see Martinsson, Griffin, and Nygren's edited collection, Challenging the Myth of Gender Equality in Sweden.
} 
indictment of the social institutions that perpetrate and enable violence against women. Harriet's and Lisbeth's histories of male violence are dramatized truths, priming us to understand "justice" within Sweden's medicolegal institutions to mean the systematic silencing of women to uphold male power, thereby necessitating feminist vigilantism.

\section{Restaging Trauma and Feminist Vigilantism}

In portraying sexualized trauma as an infectious systemic pathology, Girl with the Dragon Tattoo represents revenge rape as a viable means of subverting white misogyny. Sexualized trauma is at once rooted in, and the impetus for punishing, men who hate women—a necessary feminist revision of so-called “justice.” Harriet Vanger's and Lisbeth Salander's histories of victimization by men in positions of institutionalized authority transform into feminist vigilantism, transforming these heroines' imminent vulnerabilities - from the coercive vulnerability to sexualized violence imposed on female bodies, to an empowered state of exposure - in their efforts to facilitate justice for themselves and other women victims. Their vigilantism reestablishes severed, lost connections. As this next section explicates, Lisbeth revenge rapes Bjürman to not only cause him physical pain, but to also take ownership of rape, restaging Bjürman's rapings through her gaze and making him feel what she felt—an attempted induction of empathy. Such a reading of Dragon Tattoo mediates, although certainly does not resolve, the paradox around rape that the novel and films raise by simultaneously condemning men raping women, and condoning women revenge raping men.

Lisbeth's restaging of Bjürman's rapes becomes especially apparent in Oplev's and Fincher's film adaptations of Girl with the Dragon Tattoo, through the composition 
of each rape scene. As film theorist David Bordwell explains, cinematic staging - the ways in which scenes are staged in filmmaking- "delivers the dramatic field to our attention, sculpting it for informative, expressive, and sometimes simply pictorial effect" (8). That is, through strategic camera angles, lighting, types and sequences of shots, and cutting and editing techniques, we come to understand Lisbeth's violent response to Advokat Bjürman's perpetrations as feminist vigilantism. Such cinematic techniques and choices spotlight how "[t]he faces (and the bodies), the words (and the reactions to the words), and the gestures (and the interplay of gestures) are all working together" (ibid). The three rape scenes in Dragon Tattoo-Bjürman's two consecutive rapes of Lisbeth, and Lisbeth's revenge rape of Bjürman — transform Bjürman from authority figure to helpless victim, a state of disempowerment that is in stark contrast to the liberatory vulnerability Lisbeth enacts by revenge raping him.

In Oplev's and Fincher's film adaptations, cameras are vigilante devices that both intensify and resolve our sense of threat. When Advokat Bjürman and Lisbeth Salander first meet, the camera focuses exclusively on Bjürman's face, not Lisbeth's, suggesting that their relationship is marked by an imbalance of power. The face, the part of the body we are most attuned to in film, "serves as a constantly changing theater of moods," Bordwell explains, directing how we emotionally engage with the actions on-screen (37). As the camera pans out to include Lisbeth in the shot as well, it continues to face Bjürman, revealing only the back of Lisbeth's head, foreshadowing Bjürman's use of force against the back of Lisbeth's head in both rapes: pulling her neck to his penis to perform a blow job in the first rape, and pushing her face into a pillow in the second rape to silence her screams. When the camera faces Lisbeth, however, we see nothing of 
Bjürman, foreshadowing her eventual emancipation from his authoritative clutches. Lisbeth remains seated while Bjürman stands, dramatizing his greater mobility in their initial encounters.

In Oplev's adaptation, when the camera faces Bjürman, we also see his desk in the foreground and shelves of legal encyclopedias behind him, underscoring the institutionalized authority behind what he is telling Lisbeth about controlling her finances. “'I've been appointed your guardian,' ” Bjürman condescends, “ ' and as long as I have that role, I am legally and financially responsible for you' "(Larsson 165). When the camera faces Lisbeth, her face is in shadows, suggesting that something ominous will soon happen to her that will interfere with her ability to exercise agency. When we do see Lisbeth's face in more light, Bjürman threatens her with a negative report if she persists in demanding control of her finances. Her eyes literally glisten with rage. This change in lighting underscores that what Lisbeth finds most threatening is not Bjürman per se, but the threat to her autonomy that Bjürman represents - the threat that later catalyzes her enactments of justice for herself and for other women who may become Bjürman's victims. Their first meeting ends with Bjürman asking, "Would you prefer institutionalization?.” The scene ends on a note of interruption and uncertainty. While the camera shows Lisbeth walking towards the door to Bjürman's office, we do not actually see her leaving, the camera cutting to Mikael Blomkvist telling Erika Berger, co-owner of Millennium Magazine, that he has decided to take leave from the magazine and to work on the Harriet Vanger case for Henrik Vanger. The interrupted cut here heightens the power struggle between Lisbeth and Bjürman, which, as we will soon see, results in horrific violence. 
Advokat Bjürman's and Lisbeth Salander's second meeting, to discuss Lisbeth's request for money to purchase a new computer to replace the one destroyed in a mugging - the scene in which Bjürman rapes Lisbeth for the first time- opens with Bjürman interrogating Lisbeth about her sex life, claiming that it is his duty as her guardian: "Have you ever had any sexually transmitted diseases?," he asks, along with other questions about Lisbeth's sexual history. Both Bjürman's and Lisbeth's faces are shown as they deliver their lines of dialogue, the camera matching shots. The camera then fixates on Bjürman, following him as he stands and walks around to the other side of his desk where Lisbeth sits, "to show her the statement of her bank account which she knew down to the last öre, although it was no longer at her disposal" (Larsson 220). Lisbeth remains seated, and the camera focuses on Bjürman's face head-on, and Lisbeth's in profile. Bjürman remains standing while he places her hand on his penis. In Oplev's film, Bjürman does this while saying, “ 'If you give me any trouble, I'll make sure you spend the rest of your life in a closed ward. I know all about your mental problems and violent history." " As the novel describes, Bjürman "massag[es] the back of her neck [...] let[s] one hand slide from her left shoulder across her breasts [...] put[s] his hand over her right breast and le[aves] it there" (Larsson 220). Bjürman's gesture of walking towards and towering above a seated and therefore less mobile Lisbeth reinforces her dependent status as Bjürman's ward, and Bjürman's use of physicality to reinforce his power over her.

The escalation of threat from Lisbeth's initial request for money, to Bjürman's coercive interrogation, to his stance above Lisbeth, sets us up to perceive Sweden's Guardianship Agency as the culprit enabling Bjürman to grope Lisbeth's breasts. We thereby perceive the scene's climax, oral rape, as arising from the oppression of women 
in general and of women labeled with mental illnesses in particular. Bjürman manipulates his legal authority over Lisbeth to enact rape. Rape reinscribes the pathologization of Lisbeth as crazy that has been in place since her childhood. Bjürman uses rape to attempt to coerce Lisbeth into agreeing that she is mentally unstable and therefore in need of institutional control. When Lisbeth asserts her desire for financial autonomy, stating that she " 'want[s] to have control of [her] own money like before,' " Bjürman tries to convince her that she owes him something in return (Larsson 221). In response to Lisbeth's assertion, "Bjürman gave her a pitying look," infantilizing Lisbeth and trying to persuade her that, " "First [she] need[s] to learn to be more sociable and get along with people" " (ibid). Bjürman continues by saying they will be " 'good friends' ” who must “ 'trust each other' ” (ibid). Lisbeth remains silent. " 'You're a grown woman now, Lisbeth,' ” Bjürman says, “ 'Come here' ” (ibid). “'If you're nice to me, I'll be nice to you," " Bjürman taunts as he "pull[s] her" by the neck "down to her knees with her face in front of his crotch" (222). Lisbeth notices that Bjürman "smell[s] as if he had just washed himself with soap and water," suggesting that he planned this rape (ibid). The disparity between his words, which imply that rape is a natural and necessary consequence of Lisbeth's brazen request for money, and his bodily actions of forcing her head to his crotch dramatize the sadism that his institutionalized authority enables.

Repeating the phrase, “'If you're nice to me, I'll be nice to you,' " Bjürman threatens to " "put [her] away in an institution for the rest of [her] life" " if she does not cooperate (Larsson 222). " "Would you like that?," " he asks, "wait[ing] until she lower[s] her eyes, in what he regard[s] as submission" (ibid). The fact that the narration says "what he regards as submission" signals a disconnect between Bjürman's perception of 
Lisbeth's lowering of her eyes, and Lisbeth's actual intentions to cooperate only insofar as it will get her what she wants: access to her money (ibid). Lisbeth's responding action affirms this disconnect: "Salander open[s] her lips and t[akes] him in her mouth," a gesture that implies she is choosing to acquiesce, to "take him in," at the very moment that rape is actualized (ibid).

After the rape, Bjürman resumes his position prior to the rape, "sitting impassively behind his desk" as if nothing happened in the interim —as if he is erasing rape (Larsson 222). He continues by telling Lisbeth to " 'sit down,' " using thinly-veiled threats to try to convince her she is his subordinate; to dissuade her from reporting him; to reinforce the authority over Lisbeth that he enacts through rape; and, to coerce Lisbeth into seeing him as the ultimate authority figure by way of persuading her that if she does not get the money she requested for a new computer, it will be her fault, a result of her pathology and her dysfunctionality. When Lisbeth returns from the bathroom, where she scrubs her mouth out with soap after the blowjob, Bjürman says, “'You're grown up now, aren't you [...] you also need to be able to play grown-up games [...] I don't think it would be a good idea for you to tell anyone about our games. Think about it—who would believe you? There are documents stating that you're non compos mentis [not sane]. It would be your word against mine. Whose word do you think would carry more weight?' " (222-23). An expert in rationalizing his own behavior, a common characteristic in perpetrators, Bjürman tells Lisbeth that they will be " 'good friends;'” that she was “ 'smart to come and see [him];'” " and that she " 'can always come to [him]' " (223). Bjürman's threat to use Lisbeth's medicolegal records to reinstitutionalize her highlights how entire systems, not just individual perpetrators, enable violence against women. Case 
in point: the falsified reports from Dr. Teleborian made their way into the hands of a sadistic legal guardian, Advokat Bjürman.

Lisbeth matches Bjürman's feigned ignorance, acting as if nothing happened by repeating the request she made prior to the rape: " 'I need 10,000 kroner for my computer,' she sa[ys], precisely, as if she were continuing the conversation they were having before the interruption" (Larsson 223). Lisbeth's statement unnerves Bjürman, because he expected that rape would terrorize her into submission. Lisbeth's response here exemplifies how she is already starting the process of restaging rape, of putting Bjürman in the position of experiencing his own tyranny. He "raise[s] his eyebrows" and thinks to himself, "Hard-nosed bitch. She really is fucking retarded. He hand[s] her the cheque [...] This is better than a whore. She gets paid with her own money" (223). In Fincher's film, this first rape scene ends with Lisbeth at home, sitting on the floor of her apartment. As the camera zooms in on her face, it turns upside-down, foreshadowing a reversal (of victim and perpetrator roles) that will ensue as a result of Bjürman's oral rape.

In addition to the particular uses of cameras to film rape scenes Girl with the Dragon Tattoo, Lisbeth herself uses camera technologies as instruments to enact feminist vigilantism. After the first rape, Lisbeth decides to make another appointment with Bjürman to ask for money, anticipating that he will force another blowjob on her. She plans to hide a wide-angle digital video camera in her rucksack to record Bjürman raping her so that she can use the video as leverage for securing her independence from Bjürman and the guardianship system as a whole. Lisbeth's and Bjürman's next encounter, in which the second rape takes place, begins with Lisbeth phoning Bjürman to arrange a 
meeting — a bold, proactive response to Bjürman's oral rape. The camera faces Lisbeth head-on while she speaks to him. Her mouth, one of "the most informative zones" of the face Bordwell says, is clenched, illustrating that the cordiality Lisbeth is feigning on the phone is her attempt to catch Bjürman off guard and manipulate him (37).

When Lisbeth arrives at Bjürman's apartment where they've arranged to have this meeting, Bjürman again stands while Lisbeth leans against Bjürman's bed to display (this time, calculated) submission. To her surprise, Bjürman's violence escalates. He handcuffs her, ties her to the bed face down, and tears off her clothes: “ 'I'm going to teach you how this grown-up game is played. If you don't treat me well, you have to be punished. When you're nice to me, we'll be friends.' He sat astride her [...] 'So you don't like anal sex,' he sa[ys] [...] Then she felt an excruciating pain as he forced something up her anus " (Larsson 250). Assuming an even more dominant position of not just standing above Lisbeth, but mounting her from behind while she cannot move, Bjürman anally rapes Lisbeth. Unlike the first rape scene, in which the camera moves away from Lisbeth to focus on Bjürman's facial expression of pleasure while Lisbeth performs fallatio, in this second rape scene, the camera alternates between Bjürman's demonic expression of pleasure and Lisbeth's expression of pain, ultimately resting on Lisbeth's face. In Oplev's adaptation, the camera spotlights Lisbeth's face from the position of the floor, looking up, moving away from the physiological site of rape, Bjürman's penis thrusting into Lisbeth's anus, towards Lisbeth's face, her own underwear gagging her mouth, her struggle to scream and break free of the handcuffs and bindings Bjürman has wound around her wrists and ankles, Bjürman's hands as he pushes her head down, and Lisbeth finally screaming, without restraint, as she finally gets the gag out of her mouth. 
The camera's focus on Lisbeth's face suggests a repositioning-from Bjürman to Lisbeth - at the heart of this scene. This repositioning encourages viewers' identification with Lisbeth's trauma, taking the spotlight away from the specific act of rape and towards the psychological terror it temporarily produces in her. The scene cuts out in the middle of Lisbeth's scream, to the bridge to Hedeby Island where Mikael Blomkvist is assembling evidence for the Harriet Vanger case. This cut underscores how Lisbeth's fate is bound up with the investigation of Harriet's disappearance. When the camera cuts back to Bjürman's apartment, Lisbeth is dressed and gathering her things to leave. We do not see how the rape ends, or how Lisbeth gets out of the handcuffs and bindings. The camera follows Lisbeth as she staggers home. In Fincher's film, the camera then momentarily rests on the image of the check Bjürman wrote for Lisbeth, centering the memo line, which reads, "for food." This image underscores how rape is the price the medicolegal system has set for Lisbeth's basic daily sustenance, for survival—by way of granting authority to Dr. Teleborian, Advokat Bjürman, and other male institutional figures who try to keep Lisbeth down. We then see Lisbeth's back as she squats in the shower to wash. Blood and water stream down the dragon tattoo on her back, Lisbeth's and the entire story's identifying feature — a symbol of feminist vigilante justice that arises from what Lisbeth rightly identifies as Bjürman's "systematic brutality" (Larsson 252).

In the third and final rape scene in Dragon Tattoo, Lisbeth completes the restaging of Bjürman's previous two rapes. When Lisbeth comes to Bjürman's apartment for their next appointment, the disparity between Bjürman's perception of Lisbeth as dim-witted and crazy, and Lisbeth's actual agency, is palpable. Bjürman greets Lisbeth 
with feigned empathy: “ 'I suppose it was a bit rough last time [...] You looked a little subdued" " (Larsson 254). In response, Lisbeth smiles “a crooked smile," and Bjürman experiences "a sudden pang of uncertainty" (ibid). When they reach his bed, "Bjürman realize[s] something [i]s amiss. She was leading him to the bed, not the other way around" (255). Lisbeth tasers Bjürman with 75,000 volts; he drops to the ground.

In both Oplev's and Fincher's films, the scene then cuts to Mikael reviewing photos from the Children's Day parade in Hedestad, the last place anyone claims to have seen Harriet Vanger before her disappearance forty years ago. These photos "crack open" the case and eventually, will provide the clue that links Harriet's disappearance to Martin Vanger (Larsson 295). When the camera returns to Bjürman's apartment, Bjürman is lying bound on his bedroom floor, his mouth taped shut while Lisbeth lights a cigarette, putting the video of Bjürman anally raping her into his DVD player. Bjürman lies in a fetal position, symbolizing how the revenge rape that follows is an attempted induction of empathy — a repositioning of Bjürman's body to be reborn through rape, to reform his sadism, and to stop raping Lisbeth and other women. To be clear, while Lisbeth's attempt to induce empathy in Bjürman by way of revenge rape is certainly justified, given Bjürman's brutality and Lisbeth's extended history of victimization, revenge rape is feminist vigilantism, not justice. Agency in this scene is fully reversed: Lisbeth stands, pacing, while Bjürman struggles to get free. Then, she shoves a large anal plug into Bjürman's anus, the veins on her neck popping out as she does so-a parallel to the demonic expression on Bjürman's face when he forces his penis into her anus in the previous rape scene. " 'You enjoy shoving things inside people, is that it? [...] Without a 
lubricant, yes?,' " Lisbeth taunts—a revision of Bjürman's “ 'so you don't like anal sex' " remark (Larsson 258).

Lisbeth leaves the plug inside Bjürman's anus while she speaks to him, assuming the same manipulative, confident tone of voice Bjürman uses in the previous rape scenes. Lisbeth begins by saying, “ "This video shows you raping a mentally handicapped twenty-four-year-old girl for whom you were appointed guardian (Larsson 259). She coopts the institutionalized perception of Lisbeth as "mentally handicapped" to set the terms of her release: “ '[Y]ou have no idea how mentally handicapped I can be if push comes to shove [...] This is what's going to happen' "(Larsson 260). She continues: “ 'Next week, as soon as you manage to shit out that oversized rubber plug in your arse, you're going to inform my bank that I—and I alone — have access to my account' " (ibid). First and foremost, Lisbeth insists upon her initial request for financial independence, to ensure that she will never again be in the position of having to ask him for money - the request that incited the first two rapes. She also insists on no contact. " 'If you ever try to contact me again,'” "she threatens, “ "copies of this DVD will wind up in every newsroom in Stockholm' " (ibid). To ensure the efficacy of her threats, Lisbeth is very specific in her demands:

"Once a year you will turn in your report on my welfare to the Guardianship Agency. You will report that my life is completely normal, that I have a steady job, that I'm supporting myself, and that you don't think there is anything abnormal about my behavior [...] Each month you will prepare a report about your nonexistent meetings with me. You will describe in detail how positive I am and how well things are going for me 
[...] In a year or so, let's say two, you will initiate negotiations in the district court to have my declaration of incompetence rescinded. You will use your faked reports from our meetings as the basis for your proposal. You will find a shrink who will swear under oath that I am completely normal $[\ldots]$ You will do precisely everything in your power to ensure that I am declared competent." (260-61)

Lisbeth has so thoroughly studied how the legal guardian system works, she knows exactly what to demand from Bjürman. Lisbeth threatens not to kill Bjürman, which would certainly prevent him from raping her again, but to insist upon a deeper, structural emancipation for herself: release from the medicolegal system altogether. Her subsequent demands ensure that she will never be at his mercy again, because she demands that in addition to allowing her financial freedom, that he must also represent her to other authorities as stable, secure, capable, and autonomous.

After instructing Bjürman in releasing her from his and the guardianship system's control, Lisbeth kicks the plug further into Bjürman's anus, reinforcing not only the violence of her revenge rape and the seriousness of her threats, but also her ability to literally fuck Bjürman over, should he fail to comply with her demands. Doing so turns Bjürman's earlier, “ 'If you're nice to me, I'll be nice to you' " manipulations into consequences. It says, "You haven't been nice to me, so I'm not going to be nice to you." In contrast to the first rape scene, when Bjürman's face is the main object of focus, and the second rape, when the camera alternates between Bjürman's and Lisbeth's faces, Lisbeth's face is the main object of focus throughout this revenge rape scene. While Lisbeth forces Bjürman to watch the video of Bjürman anally raping her, the camera rests 
on her face. When we see Bjürman again, his mouth is taped shut, his eyes water with rage, and Lisbeth is tattooing, "I AM A SADISTIC PIG AND A RAPIST," onto his stomach so he will " 'always remember [their] agreement' " (Larsson 262). This action reinforces Lisbeth's remark, “' I don't think you and I are going to be good friends,', using Bjürman's very manipulations of Lisbeth against him. The camera faces Bjürman from the ceiling, looking down — a complete reversal of the ground-up shots of Lisbeth's screaming face while Bjürman anally rapes her. The camera cuts to a hallway-view shot of Bjürman's bedroom door. It stands ajar. The camera slowly backs away, towards the front door of his apartment, while continuing to face the bedroom door. We see a trail of blood, likely Bjürman's, leftover from when Lisbeth tasered and dragged him into the bedroom.

Here, the revenge rape scene cuts to Mikael identifying Harriet's changing facial expression in the parade photos, the first clue in linking Harriet's disappearance to Martin Vanger's violence. Mikael notes that Harriet's changing expression, from contentment to distress, signals that "She sees something-someone-on the other side of the street. She reacts with shock. She contacts Vanger for a private conversation which never happens. She vanishes without a trace" (Larsson 307). The scene cut here effectively bridges Lisbeth's revenge rape of Bjürman and solving Harriet Vanger's disappearance, and securing justice for women sexually victimized by men in positions of institutionalized power. The camera cuts back to Bjürman lying passed-out on his floor, with a full shot of the tattoo Lisbeth brands him with, "cover[ing] his belly, from his nipples to just about his genitals" (263). The view of Bjürman here parallels the shot of Lisbeth's full dragon tattoo when she showers after Bjürman's anal rape. Blood surrounds his body, as it had 
surrounded hers. Lisbeth's revenge rape secures justice for other women who may be, or may become, victims of Bjürman's sadism. Lisbeth warns Bjürman, “ 'I'll be checking up on you. If I ever find out you have been with a girl again — and it doesn't matter if she's here of her own free will-if I ever find you with any woman at all...' Salander made the throat-slitting motion" (261). Lisbeth boasts that if Bjürman fails to follow her rules, he will be the one facing institutionalization, not the other way around. " I'm going to have control over your life," " Lisbeth says, and “ “[w]hen you least expect it, when you're in bed asleep probably, I'm going to appear in the bedroom with this in my hand.' She held up the taser" (ibid). Using Bjürman's own methods of coercion against him, Lisbeth recovers the freedom rape has taken from her. The camera cuts back to the hallway in Bjürman's apartment— - this time, facing away from his bedroom, following Lisbeth as she leaves out the front door. The scene ends on departure, movement, agency. When Lisbeth returns home afterwards, she immediately sits down to email Mikael with Bible passages from the Book of Leviticus, describing punishments for sinning, such as burning women alive (Leviticus 21:9), stoning them to death (Leviticus 20:27), and cutting their bodies into pieces (Leviticus 1:12). As Lisbeth figures out, these verses match exactly the manners in which multiple Jewish women across Sweden were murdered. Their cases were never solved; no one recognized the pattern. The seemingly random list of names and numbers in Harriet's diary are the names of these women, and the Leviticus verses that correspond with their deaths. These passages enable Mikael and Lisbeth to solve Harriet's case, directing them to Martin, exposing Martin as a neo-Nazi killer of women, and resulting in the discovery that while Martin did not murder Harriet, he did murder many other women and is responsible for Harriet's disappearance. He 
knew that Harriet knew; he knew that Harriet murdered their father; and, he immediately took over his father's role as an incestual rapist, raping Harriet himself.

The sequencing of Mikael studying the parade photos of Harriet, Lisbeth revenge raping Bjürman, and Lisbeth emailing Mikael with Leviticus passages connects the Mikael/Harriet and Lisbeth/Bjürman storylines, sealing the intersection of Lisbeth's and Harriet's fates. In this way, Lisbeth's revenge rape not only completes her own liberation from Bjürman, but also compels her to finally contact Mikael, who she has been secretly following since Henrik Vanger hired her, vis-a-vis Milton Security, to do a background check on him. Revenge rape catalyzes justice for multiple women, one woman's actions completing another's.

This connection is affirmed in the penultimate scene of the films. Lisbeth finds Martin in his basement, about to kill Mikael. Lisbeth clubs Martin over the head, and he staggers out to his car while Lisbeth unties Mikael from Martin's ropes. Lisbeth asks Mikael for permission to kill Martin, Mikael nods with approval, and Lisbeth cocks the gun she finds on the basement floor, then chasing after Martin on her motorcycle. She drives Martin off the bridge, his jeep tipping over and bursting into flame with Martin trapped inside. In Fincher's film, the scene closes with a shot of Lisbeth's profile, her eyes glancing down as she relocks the gun's safety catch, symbolizing that she is responsible for Martin's death, for putting an end to his violence, even though she does not actually pull the trigger.

While Lisbeth's acts of vigilantism represent strategic negotiations of what she desires (independence), and what actually is (institutionalized misogyny), they may not be construed as "feminist" per se. In fact, her actions seem to replicate the very gendered 
violence Lisbeth allegedly resists, portraying rape and murder as viable ways for women to stop male violence. As Sarah Projansky argues in Watching Rape: Film and Television in Postfeminist Culture, the ubiquitous rape representations in mainstream film and television structure how we perceive and feel about rape, becoming "part of the fabric of what rape is" (2). Projansky elucidates a representational paradox around rape, in which films and television shows at once condemn rape as a form of misogynist power and control, yet also necessitate rape "for the articulation of feminism" (21). Drawing on the example of Tom Topor's The Accused, one of the first Hollywood films to depict rape graphically, Projansky explains that the graphic rape scene in The Accused challenges the rape myth that a woman is responsible for rape if she "dresses and dances provocatively in a public bar [yet] the simple fact that [the film] includes a representation of rape contributes to the existence of rape on a representational level" and "to the existence of violence against women in media culture" (96). Projansky concludes that The Accused both challenges and impels rape in a (post)feminist culture.

Like the rape scene in The Accused, rape scenes in Girl with the Dragon Tattoo also "produce spectatorial anxiety," "resolv[ing]" it through "an independent woman character who triumphs in the end:" Lisbeth (Projansky 97). In this way, Lisbeth's revenge rape may not appear as fearsome as Bjürman's rapes because it is set up as a falling action, an aftermath of Bjürman's initial rapes, the rising action. Further, Dragon Tattoo valorizes rape because it "transforms [Lisbeth] into a powerful and independent agent who can protect herself" as well as other women (100). As psychologist David Anderegg explains in his essay, "What to Say When the Patient Doesn't Talk: Lisbeth Salander and the Problem of Silence," "[w]e admire Lisbeth because, unlike us, "who 
eventually ha[ve] to capitulate," she succeeds in defeating the authorities (75). Lisbeth's vigilantism enacts a greater feminist desire for autonomy.

\section{Challenging Traumatic Truths}

In its representation of sexualized trauma as both a product of white misogyny and a catalyst for feminist vigilantism, Girl with the Dragon Tattoo challenges the individualization and (bio)medicalization of suffering. Dragon Tattoo's representations of trauma shift the habitual focus of trauma inquiry away from the individuals who experience trauma, towards the events and networked conditions that produce it. Dragon Tattoo characterizes social institutions as profit-seeking enterprises that need radical overhaul. It calls into question the ethics and prized clinical utility of a diagnostic system that pathologizes victims of domestic violence and rape for being victimized, suggesting that diagnosing systems, not individuals, can save the day and protect women from further male violence. In so doing, Dragon Tattoo characterizes catastrophic events themselves, rather than victims/survivors of such events, as traumatized, indicating that white misogyny has robbed victims of the right to govern their own bodies.

Psychiatric diagnosis brings about Bjürman's false perception of Lisbeth as in need of institutional control, overlooking possibilities that Lisbeth's history of violent behavior is a necessary survival response, not psychopathology. " 'Keep in mind that I'm crazy, won't you?,'" Lisbeth says to Bjürman as she completes her revenge rape in the films. As this line of dialogue indicates, Lisbeth challenges the authority of psychiatric diagnosis by using the labels imposed on her since childhood to assert her own agency. Lisbeth thereby exposes the falsity of naturalized assumptions Bjürman makes about 
Lisbeth as a "crazy" (i.e., pathological) woman: stupid, weak, reckless. Lisbeth transforms victimization and pathologization into feminist vigilantism, exposing how Dr. Teleborian's original diagnosis of her as mentally ill, paranoid schizophrenic, and Bjürman's exploitation of that diagnosis by raping her, are results of male-governed institutions: the Secret Police (Lisbeth's father, Zalachenko), the guardianship system (Advokat Bjürman), and psychiatric wards (Dr. Teleborian).

Diagnosing pathology at the level of systems enables Lisbeth to stop Bjürman from raping her and other women in the future, and it enables Mikael to catch Martin Vanger and resolve Harriet Vanger's case. Dragon Tattoo portrays rape itself as an act of traumatic exposure (which I defined in Chapter Two as "experiencing or witnessing an event beyond one's control"), traumatic intrusion (which I defined in Chapter Two as "a hostile takeover"), traumatic avoidance (which I defined in Chapter Two as "conscious, strategic efforts to forget"), traumatic negativity (which I defined in Chapter Two as "an absence of presence"), and traumatic arousal (which I defined in Chapter Two as "a metaphorical alarm clock, without a snooze button, going off in the brain")—DSM-5's criteria for diagnosing PTSD in individual patients.

Rape is a mechanism of exposure, rendering Lisbeth defenseless, at first, against the sadistic will of men. Rape reveals something shameful: the white misogyny corruption Sweden's medicolegal system - its failure to protect Lisbeth and to, in fact, expose her repeatedly to the very violence that leads to the pathologization that got her dependent in the first place. In this way, rape is also marked by intrusion, a hostile takeover that interferes with Lisbeth meeting her core need for independence, breaking down her autonomy. 
Girl with the Dragon Tattoo imagines a world in which traumatized subjects do not embody naturalized PTSD symptoms but rather, a desire for righteous revengeance. Lisbeth represents a kind of new world order that is not justice per se, but that exists beyond white, misogynist corporate and institutional structures - in which violence against women is met with violence against men. Contrary to $D S M-5$ 's description of catastrophic events as inducing traumatic avoidance, Lisbeth does not try to forget what happened to her. Lisbeth does the opposite. After Bjürman anally rapes her, Lisbeth gets a tattoo around her ankle, where Bjürman had tied her up to his bed. " 'It's a reminder,' " Lisbeth insists (Larsson 254). In contrast to medicalized conceptions of traumatic avoidance, Lisbeth wants to remember and to not forget. Lisbeth rejects traumatic negativity, cognitive distortions that can cause victims to blame themselves and to internalize violence. Rape clarifies Lisbeth's and Harriet's perceptions that rape is not an isolated act, but one sustained and perpetuated by whole systems or networks of misogynists. When Lisbeth revenge rapes Bjürman, rape exercises arousal—an anxious awakening that Lisbeth undergoes as a result of Bjürman's sadistic rapes that produces an excess of violence which she then casts back onto him.

In reframing whole systems as perpetrators of violence against women, and portraying the event of rape itself as bearing symptoms of trauma, Girl with the Dragon Tattoo exposes how the trauma responses that perpetration produces (e.g., social isolation) may not so much be rooted in individual pathology as they may indicate what white misogyny has stolen from women: the ability to trust, to connect with others, and to access social services to secure justice. 


\section{Conclusion}

Demonstrating how Girl With the Dragon Tattoo conditions us to perceive sexualized violence as a systemic pathology that catalyzes white feminist vigilante justice, this chapter questions the efficacy of naturalized, medicalized individualism around trauma. Perceiving sexualized violence systemically, as Dragon Tattoo conditions us to do, enables an understanding of trauma as an institutional, in addition to an interpersonal, phenomena. Moreover, Dragon Tattoo underscores how a failure to "diagnose the system" perpetuates the very conditions in which trauma is likely to occur. Trauma is a systemic disease, a cultural experience, and the underlying social conditions that produce rape. That said, while Girl with the Dragon Tattoo rights the injustices individual characters experience, it does not resolve the institutionalized ideologies that produce these injustices in the first place. Thus in the next chapter, I investigate how systemic pathologies are challenged by collective memorialization, in contrast to the feminist vigilantism enacted in Girl with the Dragon Tattoo, in Nava Semel's hybrid Nazi Holocaust novel, And the Rat Laughed. 


\section{CHAPTER IV}

\section{A CASE STUDY OF LITERARY TRAUMA DISCOURSES: DISCURSIVE FORMATIONS OF TRAUMA IN AND THE RAT LAUGHED}

Like Stieg Larsson's Girl With the Dragon Tattoo, Nava Semel's hybrid Nazi Holocaust novel, And the Rat Laughed, discursively constructs sexualized violence against females as a systemic pathology — a mechanism of anti-Semitic misogynyuprooting trauma from the biomedicalized pathology model that The Diagnostic and Statistical Manual of Mental Disorders V(DSM-5) naturalizes. Yet while rape trauma in Dragon Tattoo catalyzes feminist vigilantism, punishing specific perpetrators and emancipating the particular women these men rape from future victimization, rape trauma in Rat catalyzes cross-generational, cross-species hybridity, holding the world accountable for bearing witness to and memorializing Nazi Holocaust trauma, and transforming victimization into the empowered vulnerability of interdependency.

Conducting a case study of sexualized trauma in And the Rat Laughed, this chapter expands upon the reconfigurations of traumatic experience, traumatic agency, and feminist vigilante justice that Girl with the Dragon Tattoo introduces. Where Dragon Tattoo represents trauma as an institutional (medicolegal) failure, Rat collectivizes traumatic experience itself, representing how institutionalized anti-Semitic misogyny enables rape, but also how the trauma brought about by rape surpasses individuals who are raped and their particular stories of sexualized victimization. Instead, Rat suggests, trauma is an always-already aggregate of subjects, narrative accounts, and 
spatiotemporalities. Where Dragon Tattoo constructs agency and justice in response to rape as feminist vigilantism enacted by singular subjects, Lisbeth Salander and Harriet Vanger, Rat illustrates how trauma demands collective responses that far exceed reparations for singular instances of sexualized violence. Moreover, like Dragon Tattoo, Rat also exposes the limitations of institutionalized justice (e.g., sentencing perpetrators to prison) and healing (e.g., psychiatric treatment for patients diagnosed with posttraumatic stress disorder). Yet rather than spotlighting how individual characters seek justice and healing by working outside of state-sanctioned systems as Dragon Tattoo does, Rat exposes the limitations of those systems by shifting responsibility onto the world for assimilating Nazi Holocaust trauma into global consciousness. To these ends, this chapter posits that Rat's representations of sexualized violence reveal the inappropriateness of (bio)medicalized conceptions of trauma; elucidate a fundamental desire among all beings for connection, community, and belonging; and underscore how trauma dramatizes the agency of vulnerability implicit in such interdependency.

\section{The Hybridization of Nazi Holocaust Trauma}

And the Rat Laughed shapes a fictionalized story about a five year-old Jewish girl who experiences parental abandonment, forced hiding, and repeated child rape, all of which are narrated into the genocidal trauma of the Nazi Holocaust. The little girl's parents send her away into hiding to escape the extermination camps. Seeking only financial gain, the anti-Semitic Catholic farming family that takes her in hides the girl in an underground potato cellar, "the pit," where she cohabitates with rats, lice, and other subterranean creatures for a full year without sunlight. The family's son, "The Stefan," 
makes regular visits to the pit to sexually abuse the little girl, while the family refers to her as a "little sinner"—a "little Jew [who] is a danger to all of us" (Semel 21-22). Just as the farming family addresses the girl with these bigoted statements instead of a proper name, Semel also never names her, referring to her as "the little-girl-who-once-was," "A Little Holocaust," or "Girl;" or, decades after the Nazi Holocaust, as "the old woman"who this chapter refers to as, Girl-As-Grandmother. In this way, Rat discursively characterizes Girl as an apparition of something that was, marked by what she has survived. Girl is a replica of greater suffering. Trauma thereby collectivizes identity, conventionally (and medically) understood as individual property, by characterizing Girl as an unnamed subject identified by and through the very structural violence that produces her interpersonally-executed trauma.

Rat's characterization of Girl as a replica of greater suffering and moreover, the absence of any proper names in Girl's story, apart from Stefan's, distinguish And the Rat Laughed within the genre of Nazi Holocaust literature. While the theme of Jewish families sending their children into hiding is by no means unique to the genre, Rat's use of experimental literary devices such as its nomenclature define the novel as a work of speculative fiction, employing supernatural and/or fantastical elements to speculate about ethics and morals in future world. Rat employs non-linear narrative and multiple narrative voices to speculate about ethics in a post-Nazi Holocaust world. What is "the truth" about the Nazi Holocaust? How do we remember and memorialize the mass suffering, without being subsumed by its terror? How can Nazi Holocaust survivors pass on their stories to future generations without reinscribing or capitulating to violence? Rearranging past, present and future, Rat compels us to read for answers to these (among 
other) questions - to satisfy our conditioned and compulsive need to know what happened - across 156 years, from when the war ends in 1943, to when future generations encounter artifacts of Girl's story in 2099. Composed in the form of stories, legends, poems, diary entries, and science fiction, each of the novel's five parts contributes a piece of what happened to Girl, configuring her traumatic story as a compilation of first-hand testimony she shares with her granddaughter decades after the Nazi Holocaust in "Part One: The Story;" the granddaughter's account of that testimony as legend in "Part Two: The Legend;" the inferences of future generations that encounter the legend in "Part Three: The Poems" and in "Part Four: The Dream;" and, the diary entries of Father Stanislaw, who takes Girl in after the war ends in "Part Five: The Diary."

\section{Collectivizing Traumatic Experience}

The hybridization of Nazi Holocaust trauma in And the Rat Laughed collectivizes traumatic experience, reconfiguring trauma as a systemic pathology that catalyzes crossgenerational, cross-species hybridity. A product of the Nazi Regime-whose totalitarian dictatorship infected Germany, spreading throughout Europe, and ultimately dividing the world into violent alliance with or opposition to Nazism - the Nazi Holocaust itself materialized global-scale systemic brutality. As historian Doris L. Bergen notes in War and Genocide: A Concise History of the Nazi Holocaust, the Nazi Holocaust resulted from far more than the tyranny of one man, Adolf Hitler, or one political group, the Nazi Party, but from multiple, intersecting sociopolitical conditions. Bergen explains: “Hitler's Nazi regime in Germany provided the spark that set off the destruction we now call the 
Holocaust," codifying anti-Semitism to justify genocide, “and World War II (1939-1945) created a setting conducive to brutality" (emphasis added, 1). That said, Bergen continues, "without certain preconditions [...] mass murder on such a scale would not have been possible" (ibid). In other words, "a substantial part of the population had to be ready to consider it desirable, acceptable, or at least unavoidable, that certain other people would be isolated, persecuted, and killed" (ibid). While Hitler played a key role in the genocide of millions of European Jews and other persecuted groups, Hitler himself was a product of German society. Compounded social conditions preexisting Hitler—such as prejudices thriving in 20th-century Europe against Judaism, people with disabilities who were stigmatized as burdens on a militarized society that relied upon able-bodied men to fight in battle, and homosexuality; the imperialism of tremendous European conquest (e.g., forced labor and destruction of indigenous communities in Africa); and, the legacy of World War I violence, which brought imperialism back home to Europe, and created massive economic and geographic wreckage in Germany—enabled the eventual Nazi Dictatorship, which cultivated a cultural climate of hatred toward groups deemed threatening to an idealized Aryan race.

As War and Genocide explicates, during and after World War I, Hitler subscribed to an increasingly pervasive belief that Jews and Marxists were responsible for Germany's loss of the war. Echoing sentiments already circulating among war-weary German soldiers, Hitler utilized what many have referred to as his natural oratory skills to bring down democracy and promote his extremist brand of nationalism, Nazism, advocating for the creation of an allegedly "pure" Aryan master race by ridding Germany of Jewish and Polish people, and other groups Hitler perceived as enemies. Drawn to the 
numerous social benefits Hitler extended to German citizens in the wake of World War I—such as his massive reduction in employment brought about by the Depression, and his promise to restore Germany to its pre-World War I greatness - many latched onto Hitler's rhetoric, which fueled a mythology of Germanic racial superiority in which northern Europeans were racially superior. This mythology, in turn, instilled a perceived need for ethnic cleansing, forming the basis of Nazi ideology, which was actually an extension of the eugenics movement already underway in the United States. ${ }^{21}$ As this trajectory suggests, the Nazi Holocaust was a product of far more than the actions of one powerful man, but also of racial myths and theories constructed by those in political power and perpetuated by the German public. Still, this is only part of the Nazi Holocaust story (Bergen).

The systemic injustices that produced the Nazi Holocaust functioned extensively outside of the ghettos, concentration camps, and other hotbeds of Nazi terrorism. As And the Rat Laughed reveals, other forms of institutionalized, racialized hatred included sexualized violence on Jewish, female, child bodies. Global warfare and interpersonal violence mutually constituted one another; global warfare enabled the rationalization of interpersonal violence as inevitable collateral damage, and interpersonal violence reinscribed the brutality of global warfare on individual bodies.

Through the act of traumatic testimony, Rat links the coercive intimacy of rape with the widespread devastation of world war. The totality of Girl's story, Girl-AsGrandmother tells her granddaughter, is, "[d]arkness, a pit, potatoes, and then the War was over" (Semel 61). World War II punctuates Girl's story. As this narrative utterance

\footnotetext{
${ }^{21}$ For more on the intersections of U.S. eugenics movements and the Third Reich in Germany, see Edwin Black's War Against the Weak: Eugenics and America's Campaign to Create A Master Race.
} 
suggests, the materiality of a singular potato cellar is, at once, called into being by armed conflict, then eliminated by the surrender of that conflict, among nations in all corners of the world. Situating The Stefan's rapings amidst World War II, Rat inscribes his perpetration into the brutality of Nazi soldiers, and inscribes Girl's victimization into the trauma of genocide. As Stefan invades Girl's body, he racializes and sexualizes her body, taunting, “Open your Jewish legs. More. Much more. A Jewish hole. That's what you are" (Semel 32). Echoing Stefan's objectification of Girl, the poem "Big - Little" expresses Girl's internalization of Stefan's anti-Semitic misogyny: “I have a big pit outside me/I have a little pit within/The big pit is mine/The little pit is the Stefan's" (100). As these passages intimate, rape replicates the coercive control of the extermination camps onto female bodies by sexualizing and objectifying the small body of a five year-old Jewish girl.

Rape, while interpersonally executed, is a tool of the Nazi "matrix of domination," a concept developed by black feminist Patricia Hill Collins to describe an "overall social organization within which intersecting oppressions originate, develop, and are contained" (227-28). As Collins' explains, matrix of domination provides a framework for understanding how racism, sexism, ageism, and other categorized forms of oppression mutually constitute one another - that is, they depend upon one another for their meaning when, in fact, they are always-already produced, perpetuated, and sustained in tandem. The Nazi matrix of domination, in particular, describes a social organization that originated, developed, and controlled a matrix of oppressions based on race, ethnicity, and religion, as well as gender, sexual orientation, ability, health, political beliefs, and other identity markers deemed threatening to the Nazi's idealized Aryan 
master race. As Rat's inscription of interpersonal trauma into Nazi Holocaust genocide elucidates, it is not possible to separate out factors of age, gender, race and ethnicity from Girl's experience of rape; they all work together to oppress her.

Rape enacts the racialized, sexualized, gendered oppression of female Jewish bodies in And the Rat Laughed. Girl experiences rape as a female in 1944-1945 Poland, a time and space that normalized gendered divisions of labor and social roles; a time of tremendous global unrest as World War II came to an end and the Soviet Union of Socialist Republics took over Eastern Europe. Girl also experiences rape as someone of Jewish descent living in hiding around the time of D-Day, a time of global resistance to Nazi Germany, of major deportations of Jewish people to extermination camps to enact Hitler's Final Solution, and during which time people discovered hiding Jewish people were shot or publicly hung (Florida Center for Instructional Technology). Additionally, Girl experiences rape as a five year-old orphan, whose small body erupts when Stefan tears through her. The poem "Colors" in Rat mirrors the hemorrhaging, genital injuries, and other physiological damages that sexual intercourse wreaks upon her infant body: "Green is what comes out of your mouth/Red is what comes out of your legs/Brown is what comes out from behind" (Semel 109). Rape induces vomit, blood, shit. Decades later, the old woman vomits as her "guts were boiling" when recalling to her granddaughter Stefan's “open your Jewish legs” taunt (32). Sexualized violence against Girl, rationalized on the basis of her female anatomy (gender), Jewishness (race and ethnicity), childness (age), and orphanhood (familial status), illustrate how the trauma such oppression produces is also an aggregate — one that cannot be understood outside of 
the Nazi matrix of domination through any singular, isolated form of oppression (e.g., sexism).

In counterpoint to the individual pathology model $D S M-5$ espouses in defining and treating mental disorders such as posttraumatic stress disorder (PTSD), And the Rat Laughed posits that Nazi Holocaust rape trauma cannot be examined or understood outside of its roots in corrupt systems. That is, rape trauma is often decontextualized and treated as an individual rather than a cultural trauma. Nazi Holocaust rape trauma needs to be understood at the intersections of rape culture and Nazi culture. Girl's very existence depends upon the "care" of Stefan and his family. Likewise, the family's existence depends upon keeping Girl alive—albeit silently, invisibly alive—in order to receive the monetary reward for taking her in. This coerced mutuality suggests a traumatic codependency in which Girl's dependency on the family for survival enables their anti-Semitism and misogyny, and their anti-Semitism and misogyny enables them to keep Girl (barely) alive.

This paradox suggests that rape trauma is a product of entrapment, in which the perpetrator becomes the protector-a phenomenon that is by no means unique to Nazi Holocaust rape trauma, and also applies to other forms of interpersonalized violence (e.g., domestic abuse). As traumatologist Judith Herman, M.D. explains, "[a]s the victim is isolated, she becomes increasingly dependent on the perpetrator, not only for survival and basic bodily needs but also for information and even for emotional sustenance. The more frightened she is, the more she is tempted to cling to the one relationship that is permitted: the relationship with the perpetrator" (81). If rape trauma is a result of entrapment, then trauma is fundamentally a relational phenomenon. The interactions 
between Girl and The Stefan, Girl and the farming family, are structured by a mutual goal of not being found out. The boundaries between the protection of hiding and the exposure of the camps dissolve.

The coerced relationality that instigates trauma in Rat shifts the habitual focus of trauma inquiry away from the model DSM-5 naturalizes, in which "the traumatic event" is the point of departure for assessing symptoms of PTSD in patients. In contrast to Girl With the Dragon Tattoo, which focuses explicitly and intently on three incidents of rape, and whose very narrative arc is contingent upon the strategic, chronological arranging of these events to right traumatic injustices, Rat largely occludes "the event" altogether, suggesting that the instance of rape solidifies, but does not solely constitute, trauma. Instead, Rat lingers in the moments leading up to, and the aftermaths of, Stefan's sordid visits to the pit, alluding to rape exclusively through metaphor and other metonymic devices.

For example, through a simultaneous foreshadowing of what Girl-NowGrandmother is yet to reveal and a flashing-back to what she dares not recount, And the Rat Laughed intimates that Girl was raped without directly stating so. Girl-NowGrandmother can describe the critical moments leading up to rape, when she railed against her parents for sending her away. She recalls that "[s]he screamed. She kicked. She broke things" (Semel 11). She pleads, "I've been good, haven't I? [...] I love you. How come you don't love me back? I won't go. I don't want to. I won't'" (ibid). The old woman can also recall how her parents "rinsed her hair with peroxide" so she would not "look too Jewish" (15). She even remembers when her entrapment begins with the farming family "lower[ing] her into a pit under the ground" (18). 
Yet when it comes to narrating what happened while she was imprisoned in the pit, the old woman's language breaks down as "the story reaches an impasse" (Semel 19). Her narration here reads, "This is all she can offer then: I was in the dark. A muddle of time. I don't know when it began or when it was over. If it was over" (20). The syntactical structure of sentences splinters; spatial, temporal descriptions become ambiguous. We are left to decode what happened. When Girl-Now-Grandmother gets to the part of the story where Stefan rapes her, she experiences a loss of control over her own experience, over which parts of the story are transmitted, and how. Here, the narration explains that "the old woman approaches the danger zone, the limits of control, the place where she would no longer be able to hold on to the story-line" (25). We are left with an ominous sound image: "The footsteps of the farmer's son" (ibid).

Footsteps signal violence approaching. Measurable increments of warning, footsteps also represent the little agency Girl can safely exercise in the pit, her ability to count: "At five she could count already. Up to ten, and one more. Coming down, closer, his legs heavy, the wooden ladder creaking. The ninth rung is shaky... But the farmer's son knows about the weak rung, and he treads carefully. She counts till she runs out of numbers" (Semel 25). The footsteps are a brink - an aural warning in Girl's environs that she understands and can process in preparation for Stefan's violence. The footsteps affirm that trauma encompasses the event (rape), but that trauma also bleeds into moments preceding the event, thereby exceeding $D S M-5$ 's rendering of trauma as an event-based pathology. Trauma is anticipation, hoping that rape will not happen, and then experiencing that hope dashed, time and time again. Rape solidifies, but does not solely 
constitute, trauma. Rape, as feminist philosopher Claudia Card puts it, is a "terrorist institution" in which the threat of rape is ever-present in the background.

The footsteps could stop short, stumble, turn back. But they never do.

Immediately following Girl's anticipatory counting “to ten, and one more," Stefan directly addresses Girl, signaling his arrival in Girl's pit—both the pit of her potato cellar home, and the vaginal pit of her female body. Stefan utters, "Jewish skin, so soft, so smooth. Jewish undies. Don't you dare open your Jewish mouth, or I'll kill you" (Semel 28). So The Stefan commences his reign of terror with coercive affection- "so soft, so smooth" - marking Girl's body as Jewish, hated, other; as deserving of rape. Her skin is Jewish skin, the skin of the people his family abhors; the skin of the people the Nazis are trying to "exterminate." Skin is particularly symbolic of hate in the context of Nazi Holocaust historiography, as Nazis made decorative lampshades out of the skins of Jewish people murdered in the camps. By reducing Girl to skin, the text insinuates genocide.

In immediately following Stefan's derisions about Girl's skin, his “Jewish undies” remark intensifies the insinuation of genocide. Underwear is a material skin for covering and protecting genitalia. By labeling her underpants "Jewish," Stefan insinuates their otherness as well as his ownership of that otherness. Every object touching the hated body becomes hated. By the time he forcible penetrates her body, Stefan has reduced the little-girl-who-once-was to an object. Labeling parts of Girl’s body “Jewish” enables Stefan to perceive his violence as an invasion not of a human being, but of an object of hatred. Racializing every part of Girl—skin, clothing, vagina — represents a first step toward rationalizing violence. 
Stefan also labels "Jewish" her mouth, an opening from where voice emerges, as if to threaten that should her voice—-her humanness—emerge, she will die. While indicative of his own sadistic brutality, Stefan's threat also indicates the too-present possibility of Girl's voice, her agency. When Stefan speaks again, he invades the very place where voice emerges, the mouth: "I'll stick it in your mouth. Swallow it. And again, swallow it. Always swallow" (Semel 30). His literal act of oral rape completes his symbolic annihilation of Girl's source of agency and resistance. Should her voice emerge, Stefan may be found out, which could mean he can no longer experience the sadistic pleasure he derives from raping Girl. Stefan needs to continue raping Girl to validate his own power.

Throughout the novel, Stefan's character relies on self-aggrandizement and deception. His parents coddle him, and have "all kinds of nicknames for him. Stefcho. Stefaniu. Stefanek [...] [Girl] heard them calling him up above. She could detect the affection in their voices" (27). Stefan manipulates others' perceptions of him by presenting himself as a respectable, church-going Catholic: "On Sundays he went to church in his finest clothes. The village darling" (ibid). Moreover, a village girl (Janka) follows Stefan around and one day, offers up her body to him sexually. Janka says, "Let's climb down [into the pit], sweet Stefan. I'll let you touch me. You can do whatever you want to me," to which Stefan replies, "No! The village girl broke into tears, she was so disappointed. Stefan pushed her away from the mouth of the pit. She fell" (28). This passage is particularly telling of Stefan's sadistic need to exert power and control over others; he would rather rape the five year-old Girl prisoner than participate in consensual sexual activity with a girl closer to his own age who desires him. 
When Girl turns up in Father Stanislaw's church just after her release from the pit, Father sees through Stefan's guises, rightly identifying Stefan as Girl's perpetrator. Father describes Stefan as manipulative: He “[d]oesn’t say much, darting eyes. He always kisses my hand submissively, making sure his mother sees [...] Every Sunday, he would take his place at the head of the line leading to the confessional $[. .$.$] and he would whisper$ about small thefts. Trifling transgressions [...] A girl-child. He never mentioned her" (168-69). Father even works out that "[w] hat [Stefan] did to her in the dark was with the knowledge of his father and mother" (169). The entire farming family thrives on racializing and objectifying Girl in all ways, save killing her.

If the footsteps coming down the ladder threaten implosion, rape completes it. Immediately following Stefan's threat to kill Girl, the text reads, "Wrought in the dark. That's the point where the story implodes" (Semel 28). Yet it is the story and not the storyteller that implodes. The story becomes a rubble of disassembled parts, what GirlNow-Grandmother describes as "severed limbs," that can assume new forms after they are "scattered in all directions" (3-4). So where DSM-5 conceptualizes the individual as the site of traumatic event-induced implosion, And the Rat Laughed conceptualizes the story — a thing that surpasses any singular subject — as the site of implosion. The story travels far beyond the individual (Girl) who lived it. The old woman, however, remains present with the memory, and continues storytelling. "What's that there between your legs," Stefan asks (29). “Don’t you dare cry, you scum. Jewish scum. Just you wait, and I'll show you" (ibid). The page breaks, and a new section starts with, "Soaked in her own urine, in her own vomit, in her own excrement. Hemorrhaging herself” (ibid). Rape is the break. Stefan's threatening footsteps precede, and Girl's hemorrhaging body proceeds, 
rape. The bookends of "before" and "after" tell us what dwells in the middle. The scene cuts out with Stefan's threats, which imply rape, "I'll show you" what's "between your legs," picking up again with Girl's body dislodging and discharging urine, vomit, excrement, and blood.

While recalling rape threatens, but may not actually succeed in, unraveling the old woman's story, it suspends the chronology of her testimony. This rupture signals the traumatic effects of rape but, in conjunction with being torn from her parents only to be abused by her guardians, also represents the futility of trying to pinpoint a precise moment (i.e., an event) of becoming traumatized. In one sense, the narration of rape as "the limits of control" points to the vast amount of shame rape survivors can experience when they finally share their stories. In another sense, the limits of control illustrate how rape steals agency. If rape has the potential to unravel Girl's story decades after it occurred, as the language of "danger zone" suggests, then sexualized trauma becomes far more than a singular life threatening event experienced by a single survivor, but a chronic, boiling-beneath-the-surface terror that can be transmitted simply by voicing it.

In the logic of Western mental healthcare, Girl's association of Stefan's footsteps with danger and moreover, her experience of recalling them as disorienting, exemplifies one of DSM-5's PTSD symptoms: traumatic avoidance. Just before she arrives at "the danger zone," the old woman recalls that up until now, when her granddaughter insists on hearing her Nazi Holocaust story, she has repeatedly put off telling it because "it always seemed as if the story could endanger the offspring and maybe even jeopardize the chain of birth-giving" (Semel 25). For Girl-Now-Grandmother, the story is an organic entity whose agential capacities far exceed her own. To tell the story is to unleash its 
"otherworldly powers" onto her family, such that shame, denial, and rejection threaten "the chain of birth-giving" (ibid). Yet in contrast to DSM-5's conceptualization of traumatic avoidance as a survivor's efforts to avoid reexperiencing traumatic events by avoiding triggers, the old woman's traumatic avoidance may instead reflect an effort to avoid harming others (i.e., to infect her family line with her story of trauma).

"The Poems" of And the Rat Laughed confirm the eventlessness of trauma that Stefan's footsteps imply. For example, the poem "Lullaby" alludes to the ideological systems that enable rape. "Lullaby" solidifies the genocidal implications of Stefan's sordid visits to the pit, doing away altogether with a personalized account of rape. "Lullaby" reads like cultural folklore, opening with the line, "Once upon a time/There was a little Jewish girl," and shifting the story that follows out of the specificity and materiality of the real, into the realm of the fantastical (Semel 110). Yet as the poem progresses, the language brings us back to specific imagery from the old woman's testimony in the first part of the novel. The little Jewish girl in the poem is described in identical terms to Stefan's descriptions of Girl's Jewish skin and Jewish mouth: “And she had/Little Jewish hands/And little Jewish eyes/And a little Jewish mouth/And a little Jewish body/And a big hole" (ibid). The grandiosity of fairytale, and the specificity of Girl's experience merge, through lullaby. Rendering each part of the body Jewish and thereby an object, a receptacle — "a big hole" - this lullaby does not rock the body to sleep but rather, prepares it for rape.

"Lullaby" configures traumatic memory as a collective, imaginative act of passing down Nazi Holocaust stories from one generation to the next. The poem's juxtaposition of whimsical fairytale form with the theme of child rape suggests that the Nazi 
Holocaust—and more specifically, what psychoanalyst and Nazi Holocaust survivor, Dori Laub, M.D., calls the "coercively totalitarian and dehumanizing frame of reference in which [the Nazi Holocaust] was taking place"-naturalized the sexual exploitation of child bodies (66). "Lullaby" thereby calls upon the remembearers who encounter the poem to integrate sexualized violence into Nazi Holocaust historiography, by way of mythologizing Girl's story. The poem summons readers into being as witnesses, at the very moment when we may wish for relief from that responsibility. There is something to be said for forgetting. Forgetting enables survival. "For her [Girl], forgetting is healing," reads Father Stanislaw's diary (Semel 221). "[B]ut for the world, forgetting is the very disease itself," he continues, suggesting that post-Nazi Holocaust generations have an ethical, collective obligation to remember (ibid). Stanislaw's diary entry can be read as an overt critique of treating rape survivors diagnosed with PTSD with talk therapy to uncover the story; the very aversion to traumatic triggers that signals dysfunction in $D S M-5$ provides relief for Girl. Yet Stanislaw's entry also suggests that forgetting causes the violence Girl desperately needs to forget. Forgetting can at once heal and infect, depending on the one doing the forgetting. Rape survivors can forget, and heal. But when witnesses forget, they enable violence. The novel's configurations of traumatic memory as both/and suggests that traumatic experience necessitates global transmission to not only relieve survivors' pain, but to also prevent the horror of holocausts from happening again. In this way, the novel discursively constructs rape as an interpersonal encounter, at once enabled by larger social conditions and calling for global witnessing. We may despise Stefan, just as we may despise white misogynists Advokat Bjürman and Martin Vanger in Girl with the Dragon Tattoo. Yet unlike Dragon Tattoo, Rat conditions us to 
conceptualize justice as exceeding the exposure of individual perpetrators; justice must interrogate the global-scale systemic malfunction of the Nazi Holocaust that makes Stefan's perpetrations both possible and even desirable. Denial is a collective choice that produces violence, Rat suggests. Our silence, our passive standing-by, our failure to be disturbed and to allow that disturbance to stir us to action enable genocide.

Girl-Now-Grandmother dislodges post-generations from our comfort zones of complacency and silence. Her desire to set the record straight, shored up by her aging body (she is in her sixties, in 2009 Tel Aviv, when telling her granddaughter her story), propels her to speak despite the risks of transmission. As time passes, the number of Nazi Holocaust survivors who remain living dwindles. Potentially the story’s last living witness, the old woman realizes that if she does not speak, no one will; that "[s]he cannot afford to let the story disappear as if it never happened" (Semel 5). If she does let it disappear, the story will "disguise its own ugliness" and "turn into something completely different" (ibid). Girl's act of telling is an act of asserting what agency she has left—one that calls future generations into being as, what Semel calls, "[r]emembearers $[. .$.$] those$ who have the traumatic event registered in their consciousness without actually having experienced it: the second circle of witnesses to the violent experience" (150). Girl's story catalyzes remembearer witnessing.

Coupled with Semel's conversion of the word "rememberers" into "remembearers," the threat trauma poses to "the chain of birthgiving" suggests that traumatic memory is an intergenerational passing-along of embodied Nazi Holocaust knowledge. Semel's language illustrates how transmitting traumatic memories threatens to replicate suffering, not new life. So the disorientation that "the danger zone" 
phraseology implies is not so much an indication of dissociation, an individual pathology, but a recognition of our fragile interdependency. Further, Girl's act of telling validates that she did indeed endure rape, "[b]ecause once she lets go of [the story], it will be told differently. People will add things, leave things out, twist it out of shape. And all she has to go by is her own version, her own inadequate best" (Semel 6). The old woman's insight into how stories change as they are told and retold drives her to finally "give birth to" what happened for her granddaughter — so that the story loses its power to consume and destroy her; and, so that others can take on its burdens and fold it into larger narratives of Nazi Holocaust historiography. In this way, Rat suggests that traumatic avoidance bears witness to our fundamental desire for connection, community, and belonging.

The fact that Girl-Now-Grandmother approaching rape in her storyline coincides with Stefan's footsteps approaching the pit—and that both approaches are mutually cast as catastrophic (the storyline spinning out of control, the Stefan's footsteps descending into the pit) - suggests that the enactment of rape is a pinnacle or confirmation of trauma, compounded by Girl's loss of her parents, and the abuse from the farming family coercing her into Catholic prayer, slapping her across the face, and calling her a "little sinner" (Semel 22). Moreover, And the Rat Laughed encompasses multiple decades beyond and varying degrees of separation from "the event" of the Nazi Holocaust, suggesting that the events of rape and of the Nazi Holocaust are not so much beginnings or starting points of trauma, but rather, middles: tangible eruptions of violence occurring along a spectrum of ever-widening and -receding political, cultural instabilities. Eruptions, then, can be understood as material expressions of pathology, of dis-ease 
within an amalgam of interrelated histories and ideologies. Traumatic experience, the novel suggests, may be less about specific occurrences and more about what and how those occurrences signify.

\section{Functionalizing Trauma Responses}

Just as And the Rat Laughed collectivizes traumatic experience by way of assimilating Girl's story into global narratives of Nazi Holocaust historiography, and bypassing the specificity of "the event" of rape altogether, it also reconfigures the trauma rape produces as a functional, healthy attempt to survive genocide. Such reconfiguration suggests that trauma may be more effectively diagnosed and treated if its symptoms are perceived as agential, not necessarily (or exclusively) as signs of victimization. DSM-5 fails to account for how patients navigate traumatic events through whatever means possible. Thus, PTSD symptomatology may better describe the limited range of resources survivors may have for responding to rape in certain sociocultural settings than it describes individual pathology. In the Nazi matrix of domination, social mobility and autonomy are subsumed by racialized, xenophobic, gendered manipulation and indoctrination, such that Girl's options for surviving the pit may be limited to a kind of surface acquiescence to violence - an avoidance of fighting back or fleeing, and to instead, make herself as silent and invisible as possible so that Stefan will no longer recognize her as human.

In constructing rape trauma as generating healthy attempts to survive, And the Rat Laughed calls into question the efficacy of DSM-5's individual pathology model for understanding trauma, particularly in global settings where rape is a tool of corrupt, 
genocidal dictatorships. ${ }^{22}$ Rat thereby challenges the very medicalized concept of dysfunctionalism itself. For Girl, PTSD symptoms that might otherwise mark her as pathological actually signify a high level of functioning. Neglect, malnourishment, and rape sever Girl from human connection, and she experiences loneliness as a physiological "aching to be hugged" (Semel 21). In response, Girl tunes into other sources of connection and relationship, aligning her own bodily rhythms with those of the nonhuman organisms cohabitating the pit with her. While listening to this part of Girl-NowGrandmother's testimony, the granddaughter writes in her notebook, "the smell of underneath the earth stuck to the little girl, and she became blacker and blacker and darker and darker" (87). The earthy smells and the blackness sticking to Girl's body signify agency and intention, as confirmed by Girl-Now-Grandmother's testimony that "[h]er darkness is not about a lack of light nor even a contrast with light. It's a subcutaneous substance that has mass and weight, and has managed to defy the laws of nature and work its way through every barrier in the human body" (19). Girl's darkness permeates the body, takes up space, and rejects racialized constructions of darkness as a superficial marker of skin color, as a lack or absence of whiteness. Thus when Girl-NowGrandmother says that "[d]arkness was what she could feel [and that] [d]arkness even had a taste," we can understand her blackening, darkening body as agential resistance to the anti-Semitic misogyny of the Nazi Holocaust (72). As Girl's body enmeshes with the subterranean environs, her needs for relationship and connection propel her towards the safety and protection of the non-human, non-white beings with which she cohabitates.

\footnotetext{
${ }^{22}$ For more on how sexualized violence is a war crime tied to colonization and genocide, see the United Nations' Outreach Programme on the Rwanda Genocide and the United Nations.
} 
Sensorial hypervigilance, an enhanced state of sensory sensitivity and an adaptive response to trauma, sustains Girl. In contrast to hypervigilance, a psychological state of heightened sensitivity often associated with PTSD, sensorial hypervigilance underscores the role that the senses play (as distinct from the psyche alone) as they work together to perceive and ward off threats. Girl's enmeshment with the subterranean earth, absorbing its smells and colors as her own, is not an escape from trauma, but a reconfiguration of it. Where DSM-5 configures hypervigilance as dysfunctional—a marker of traumatic arousal, a cognitive-emotional agitation that can produce disruptive or aggressive behaviors towards oneself and/or others - Girl's sensorial hypervigilance enables a transformation of her neglected, violated, racialized, gendered body into a source of protection. So Rat challenges us to consider Girl's sensorial hypervigilance not as a psychic break or a split between mind and body induced by trauma, as it is conventionally understood, but as a form of bodily resistance and an epistemic resource for survival that provides additional information about what happened (i.e., the traumatic event). Where $D S M-5$ characterizes the mind/body split as dissociative and therefore dysfunctional, And the Rat Laughed characterizes the split as generative.

Sensorial hypervigilance enables Girl's body to work with and survive through the earth body. Darkness prompts a deepening of her sensory perceptions, "which had grown sharp almost instantly [...] grasping the subterranean movement. The rotting of the potatoes. The slow progress of the roots. The groaning of the wood in the ladder leading down to the pit. The wheezing of the seeds as they fought to sprout. The drops of rain percolating through the soil" (Semel 20). These virtually imperceptible movements of potatoes rotting, roots growing, wood groaning, and seeds sprouting become vital 
reminders that the little girl still lives - that she transforms her subterranean prison into a source of protection. She does not dissolve under the weight of trauma, but rather shifts into a sensory zone beyond the human realm, becoming the surrounds in which she is held captive.

To be sure, our sensory perceptions are often diluted by our naturalized, racialized sense of distinctness - from nonhuman organisms, and from bodies of different ethnicities and cultures. Ontologies that split or separate the human/animal, white/nonwhite, dilute sensory perceptions. Western epistemologies are heavily visual, privileging sight over sound, smell, touch, and taste. As Foucault explicates in Birth of the Clinic, seeing is (conflated with) knowing - a phenomenon I discussed in Chapter Two, in relation to medical diagnoses. So Girl's attunement to her extra-visual sensory perceptions indicates her resistance not only to what is happening to her in the pit (rape), but also to larger imperialist epistemologies of racialized purity (Nazism). As cultural ecologist David Abrams explains in his eco-memoir Becoming Animal, "neither the eyes, not the ears, nor the skin, tongue, or nostrils ever really operate on their own $[\ldots]$ as we explore the terrain around us, our separate senses flow together in ever-shifting ways" (251). While Abrams' memoir constitutes an ecstatic, consensual experience of enmeshing with his surrounds, as distinct from Girl's traumatic enmeshment with the subterranean, Abrams' articulation of the sustenance that enmeshment affords enables an understanding of Girl's enmeshment as restorative, protective, hopeful. Girl's process of enmeshing with the subterranean is one of reintegration that affirms the fundamental interdependence of all beings — a stark contrast to states of isolation and separateness that traumatic experience is thought to induce. In this way, "tak[ing] up less and less space 
[and] behav[ing] like a perfect subterranean creature," as Girl does, is not so much about letting go of the cruel human world in favor of a utopian harmony with nature, but about a strategic aligning of the senses to strengthen the body's ability to detect and respond to danger (23). Enmeshment allows her to hear the unhearable, to detect the undetectable. In becoming animal in this traumatic way, Girl preserves the otherness (darkness) that Stefan tries to rape out of her. To absorb the subterranean into and underneath the skin is not a sign of complacency, a resigned acceptance of the violence of the pit, but rather of community and survival transgressing the human realm. Despite all efforts from the outside world to annihilate Jewish bodies, Girl remains.

To perceive her world through the senses, as Girl perceives the pit through smell, touch, vibration, and sound, is to bind herself to a community of nonhuman organisms. To cultivate community through the senses is to survive. As Abrams puts it, "Sensory perception... is the silken web that binds our separate nervous systems into the encompassing ecosystem" (134). From an anthropocentric perspective-one that is naturalized by the medical discourses of $D S M-5$, as well as the genocidal discourses of Nazism — earth is "a subordinate space;" it is "a sinful plane, riddled with temptation [...] a menacing region needing to be beaten and bent to our will" (6). The farming family assumes that the potato cellar is punishment, "a subordinate space" for a subordinate Jewish body. Girl, in becoming animal, effectively redeems the cellar to its rightful place of existing with, providing for, sustaining life. Transforming the pit from prison to "encompassing ecosystem" ensures that it is a vaster space than any individual (e.g., Stefan) or group of people (e.g., the Nazis) can control. Thus the novel's shaping of Girl's sensorial hypervigilance as a process of becoming animal reverses the human 
tendency to "cut our lives off from the necessary nourishment of contact and interchange with other shapes of life" (7). In the future world of 2099, when the Girl \& Rat myth has been distributed across the world wide web, an unidentified person who encounters it online intuits that s/he must return to the site of trauma "to recreate the right order and cleanse the girl's name" (134). This returning to transform is bound up with sensorial hypervigilance, the enmeshment of human and animal, as the witness says, "I must return to the pit ... Need to go deep down" (135). One might go as far as to say that Girl beckons the world to reconsider such posthuman connection as the source of surviving and healing trauma.

Girl's sensorial hypervigilance is epistemically rich, signaling intelligence that exceeds the intellect—what feminist, cultural theorist, and queer theorist Gloria Anzaldúa describes as la facultad, or "the capacity to see in surface phenomena the meaning of deeper realities $[\ldots]$ an instant 'sensing' $[\ldots]$ arrived at without conscious reasoning" (60). While the mind (the psyche) is the source of both suffering and recovery, according to the logic of DSM-5, the body is that source in And the Rat Laughed. Girl responds to the pit by "shrinking and shriveling, absorbing the darkness into her... tak[ing] up less and less space" (Semel 23). While this description suggests diminishment, a sign that Stefan, the farming family, and the Nazi regime may be succeeding in annihilating her, it also suggests adaptation. In this communion, Girl (like Abrams) becomes we, "entirely a part of the animate world whose life swells within and unfolds all around us" (Abrams 3). From a sensory perspective, there is no separation between bodies and the world within which they move and breathe. "Individuality" and "autonomy" are illusions that seduce us into believing we are separate and therefore more or less powerful, more or less 
anything than other beings. These illusions, Rat suggests, are the real traumas. In learning "to behave like a perfect subterranean creature," Girl transcends separation, transforming the darkness of the pit that imprisons her into a source of companionship to transcend human perpetrations of rape and genocide.

However, as Abrams warns, it is not enough for us to psychically connect to other organisms (i.e., animals), "calling to the creature in one's imagination" (239). Rather we must do what Girl does, and "summon [the creature] bodily [...] unbind the human arrangement of one's senses, and those of any humans watching, if the animal is to feel safe enough to arrive in our midst" (ibid). In other words, to summon rats and other subterranean creatures to her side, Girl must become rat herself. We might say that Girl's "mimetic entrance" into the subterranean earth is a sign that traumatic experience does not so much shatter connection and sever relationships, but that it rearranges our anthropomorphized, racialized understanding of connection and relationship as established through the sharing of skin (color). While Girl's becoming animal is traumatic, the body's instinctual drive to survive through whatever means necessary, in contrast to Abrams' ecstatic mimetic entrance, the end result is similar: an expansion of what connection and relationship mean. Since relationships with other humans have been rendered impossible by the Nazi Holocaust, Girl's mimetic entrance links her fate to the fates of the subterranean creatures that will survive and propagate well beyond the Nazi Holocaust, as well as other human-made catastrophes. Becoming animal, concretizing our innate interbeing, heals trauma. Becoming animal is not disassociation, but reassociation. 
Later in the novel, when Father Stanislaw takes Girl in after the war ends, his tuning into Girl's sensory experience signifies solidarity, protection, and care. Girl's sensorial hypervigilance transfers traumatic knowledge to the priest. In his diary, he writes: “All night long I watch her, contorted in her strange position — half lying, half sitting. Protecting every part of her body, trying to keep from being noticed" (Semel 169). Closely observing Girl's body enables Father Stanislaw to intuit her story, as in when he acknowledges that, "[e]ven $I$ know full well what parts of the body tore through this child. My body has such a part too," when he first encounters her "[e]maciated, disheveled" body with "rags sticking to her torn flesh" (166). Witnessing Girl's enactment of "becoming animal" through the eyes of Father Stanislaw confirms the reading of her earthly enmeshment as a survival mechanism. Her contorted position signals that her body remembers the traumatic experience, whether or not the mind does. Yet her position also signals that becoming animal restores hope. He notes, "For the past few nights she's been taking apart the wooden floorboards and digging in the dirt underneath," as a rat might burrow into the ground (187). "And although I cannot fathom why she does this, I feel a strange sense of relief," he goes onto write (ibid). In other words, Father Stanislaw senses - that is, he exercises Anzaldúa's la facultad and does not just know intellectually, but bodily as well—that sensorial hypervigilance signals a kind of traumatic functionality that enables survival and rejects the bigotry of a warring human world. Girl's bodily comportment and habits are artifacts of trauma and survival. When read in relation to Abrams' foray into becoming animal, Girl's sensorial hypervigilance becomes more than mere self-preservation in the face of particular lifethreatening events (rape, the Nazi Holocaust). Sensorial hypervigilance also constructs 
the traumatic body as a crucial site of preserving and transmitting memory, even or especially when the mind rejects it. The traumatic body becomes a site of restoration. As Abrams explains, the human body is anything but singular,

[L]ess a self-enclosed sack than a realm wherein the diverse textures and colors of the world meet up with one another. The body is a place where clouds, earthworms, guitars, clucking hens, and clear-cut hillsides all converge, forging alliances, mergers, and metamorphoses... the body is itself a kind of place — not a solid object but a terrain through which the larger valleys and plains of the earth, open to the same currents, the same waters and winds that cascade across those wider spaces... a sensitive threshold through which the world experiences itself... Sometimes the world's textures move across this threshold unchanged. Sometimes they are transformed by the passage. And sometimes they reshape the doorway itself. (229-30)

When embraced as "a sensitive threshold through which the world experiences itself," the sensorially hypervigilant body becomes a place of convergence, reshaping the world through trauma. Traumatic experience travels through the body, reorganizing how its parts work together, how it interacts with the surrounds, and how the body itself is fundamentally a nomadic, infinite space whose boundaries are highly permeable. To rape one female Jewish body is to rape all such bodies; to heal one raped female Jewish body is to heal all such bodies. In this way, Girl's "digging in the dirt" (and Father's subsequent sense of inexplicable relief) is an enactment, an affirmation, of interdependency, connection, and re-emerging back into the human world above ground. 
And the Rat Laughed thus configures the body as traumatic testimony, which suggests that the body may prove a more useful site of traumatic intervention than the psyche. A Little Holocaust's body is a living testimony to sexualized Nazi violence, and to how traumatic experience can catalyze reassociation rather than dissociation, connection rather than separation, and adaptation rather than dysfunction. Such a positioning of the traumatic body as witness extends trauma theories that position the body as a storehouse of memory. As neurological traumatologist Bessel A. van der Kolk claims, "the body keeps the score" when the psyche is overwhelmed and shuts down during traumatic experience. Yet where van der Kolk conceives of the traumatic body as a place where memories of a particular event are stored when the psyche rejects them, Semel's novel conceives of the traumatic body as a conduit for traumatic memory. Girl's body stores the memory of "what happened," but also the connections, re-associations and adaptations that what happened sets in motion. Further, her body communicates to Father Stanislaw what and how she survived. Girl's traumatic body is a site of reorganizing memories by way of transferring them onto others- for instance, onto Father Stanislaw_-releasing the individual body from the burden of carrying the trauma alone. In this way, the traumatic body catalyzes connectivity, dramatizing the ways in which traumatic experience rearranges our very understanding of connectivity. In functionalizing trauma responses, dramatizing how trauma affirms our fundamental interdependency, And the Rat Laughed reconceptualizes dysfunction itself, challenging individualized, pathological connotations associated with a term typically used in psychiatric settings to describe unhealthy behaviors and attitudes, and a general inability to function normally. As Chapter Two of this dissertation explicates, Western 
psychiatry configures dysfunction as the root of mental illness - "a syndrome characterized by clinically significant disturbance in an individual's cognition, emotion regulation, or behavior that reflects a dysfunction in the psychological, biological, or developmental processes underlying mental functioning" (emphasis added, American Psychiatric Association 20). The American Psychiatric Association (APA) thereby individualizes mental health, locating functionality in "psychological, biological, or developmental processes," rather than in sociocultural or institutional ones. As Ethan Watters notes in analyzing the influx of Western mental healthcare workers to Sri Lanka in the wake of the 2004 tsunami, DSM-5 universalizes its diagnostic category of PTSD, propagating the assumption "that the psychological reaction to horrible events is fundamentally the same around the world" (68). In actuality, Watters elucidates, By and large Sri Lankans didn't report pathological reactions to trauma in line with the internal states (anxiety, fear, numbing, and the like) that make up most of the PTSD symptom checklist. Rather Sri Lankans tended to see the negative consequences of an event like the tsunami in terms of the damage it did to social relationships. Those who continued to suffer long after a horrible experience [...] were those who had become isolated from their social network or who were not fulfilling their role in kinship groups. In short, they conceived of the damage done by the tsunami as occurring not inside their mind but outside the self, in the social environment. (91)

If traumatic events are experienced as social dis-ease rather than psychological pathology, as Watters' case study and representations of trauma in And the Rat Laughed 
suggest, then the very concept of (dys)functionality that allegedly produces PTSD (among other mental disorders) is anything but universal.

For survivors of sexualized violence such as Girl, assuming the universality of responses to traumatic events blames the victim, keeping her sick, locating dysfunction within the survivor rather than in the systemic anti-Semitic misogyny that produces rape. In this way, $D S M-5$ reinforces a false binary between individual and society, suggesting that the two function independently of one another. DSM is a Western production and as such, inherits the epistemic, ontological, and social frameworks of dominant groups. So while $D S M-5$ nods to the cultural-variability of dysfunction, conceding that what constitutes normalcy and pathology in one setting may not in another, the individualization of functionality in the manual's very definition of mental disorder can lead to diagnoses that fail to identify how social norms contribute to and manifest certain disorders. Countering such medicalized assumptions about functionality, And the Rat Laughed collectivizes dysfunction, configuring it as a product of genocidal tyranny (Nazism and misogyny), not individual pathology, closing rhetorically constructed gaps between individual and society, normalcy and pathology.

\section{Re-localizing Trauma}

The previous two sections explicated how And the Rat Laughed collectivizes traumatic experience, and configures the trauma responses rape produces as functional, healthy attempts to survive catastrophic events. Part One decodes rape in the novel as systemic brutality, a product of the Nazi matrix of domination. Decoding rape in this way underscores how perpetrators never act independently, but instead, rely (consciously or 
not) on particular arrangements of power that enable and even valorize their violence. Such decoding also underscores how victims never experience rape as singular subjects (e.g., as a woman, OR as a person of color, OR as a child, and so forth), but as multiplicitous beings, pointing up how sexualized violence requires responses that attend to how it affects victims differently depending upon intersections of ethnicity, race, gender, sexuality, age, cultural and historical context, and other contingencies, thereby necessitating interventions that start in lived experience. The novel challenges the normalized medical pathologization of rape survivors and moreover, as Part Two posits, illustrates how trauma responses typically labeled "dysfunctional" (e.g., PTSD symptoms of traumatic avoidance) actually affirm the fundamental interdependency among humans, animals, and other organisms. In this last section, I tie these threads together, showing how collectivizing traumatic experience and functionalizing trauma responses demand a revision of trauma itself: as a transmission across generic forms, time, and space- similar to Luckhurst's configuration of trauma as transmittable rupture in The Trauma Question.

In its non-linear narrative form with multiple narrators, And the Rat Laughed assembles the disparate parts of Girl's story into a whole, offering an alternative to psychiatric understandings of trauma as an individual pathology that requires interpersonal intervention (i.e., between a singular doctor or medical provider, and a singular patient). For example, the novel imagines a world that dramatizes Dori Laub's claim that the Nazi Holocaust "produced no witnesses" as "the very circumstance of being inside the event made unthinkable the very notion that a witness could exist, that is, someone who could step outside of the coercively totalitarian and dehumanizing frame of reference in which the event was taking place" (65-66). And the Rat Laughed envisions 
that the Nazi Holocaust produced multiple witnesses, with differing degrees of separation from "the event" that when listened to in relation to one another, reveal how bearing traumatic witness is not so much impossible as it is reliant upon those who encounter it to bring the story into, and sustain it in, collective consciousness.

At the very beginning of Rat, Girl-Now-Grandmother writhes in response to her granddaughter's request that the little-girl-who-once-was share her story so that she (granddaughter) can complete a school assignment on Nazi Holocaust testimonies. GirlNow-Grandmother struggles to find a starting point: "How to tell this story? [...] But maybe there's no need to tell it [...] How should the story be told? But maybe it's been told already. Leaking through in moments of distraction, forcing its way to the surface whenever she loosened her grip [...] it's as if she has no choice but to assume the role of storyteller. But she doesn't know how" (Semel 3). This oscillation between the compulsion to get the story out, and Girl-Now-Grandmother's fear of the damage voicing the story might cause persist throughout her conversation with her granddaughter. Ultimately, she presents her story as a legend, her granddaughter despairing at having an “empty notebook [...] no story, no testimony, nothing that could be used to teach the coming generations a lesson" (79). "Just a legend [the grandmother says]. Take it or leave it" (ibid). The struggle to tell materializes as a fictionalized account, a legend, that while regarded as historical, remains unauthenticated. The form of "legend" allows the littlegirl-who-once-was to pass on her experience without the risks of exposure that "testimony" suggests.

The granddaughter brings the legend to school the next day, sharing it with her teacher and classmates. The granddaughter narrates rape as an animalistic, supernatural 
phenomenon when she says that, "another human climbed down underneath the ground. Not a man-cub like the little girl, but one that had also been created in the divine image [human]. He began to bite her [...] And digging tunnels inside her" (Semel 87). Echoing the paralysis Girl experienced as a result of rape, the granddaughter explains that, "By now, this little girl was the most silent creature on earth $[\ldots]$ And then the little girl climbed out of the earth" when the war ends and Father Stanislaw takes her in (88-89). Telling the grandmother's testimony as legend displaces rape from its naturalized position as interpersonal, private, intimate, folding rape into larger narratives of Nazi Holocaust historiography.

Furthermore, experiencing her grandmother's testimony as legend transfers trauma onto the granddaughter. As if she herself becomes the little girl, scrambling her way up and out of the pit, the granddaughter confesses that when the power cuts out during her grandmother's telling of the legend, "My legs ran up the stairs. I don't know how to explain it, I don't know why myself [...] I went up in the dark and it was my own darkness" (Semel 91). Like her grandmother, the granddaughter also experiences darkness as a "subcutaneous substance" with mass and weight: "I could taste it, chew it even. That darkness got stuck between my teeth, in my throat, in my stomach, between my legs" (ibid). Also like her grandmother, the granddaughter feels compelled to vomit during this "between the legs" (i.e., rape) part of the story. "I wanted to throw up. And even when I really wanted to open my eyes I couldn't, as if something stronger than me was keeping them shut [...] I've never been so scared in my whole life. I don't know how to explain it. I kept running up the stairs" (91). As the granddaughter's account of Girl's legend suggests, the act of bearing witness transmits trauma even as the witness resists it. 
When the grandmother tries to tell her story, she reexperiences it against her will - just as the granddaughter does here. More importantly, reexperiencing is compulsory and coercive, forcing remembrance and suspending the witness's freedom to choose to forget.

This same compulsory reexperiencing of trauma recurs when an anonymous "nave@infomail.co.il" encounters the poems on the internet-poems that the granddaughter's classmates produce in response to hearing her tell the legend at school. Nave@infomail says, “The poems - or maybe they’re words that have come undone just showed up while I was surfing some housepet sites. I tried to resist at first, even tried some evasion tactics, but it was stronger than me, and against my better judgment I found myself inside" (Semel 95). In wondering whether the poems are actually poems, or "words that have come undone," nave@infomail hints that the fragments we call "lines" and the assemblages of fragments we call "poems" are more reflections of humans' conditioned and compulsive need to categorize than they are "truth." Thus, this remark suggests that trauma itself resists categorization and "truth," enforcing its own ways of telling.

Asnave@infomail continues, we learn that once read, the poems become irrevocably ingrained in those who read them: "The little girl and the rat are deep inside me now, and I can tell I'll never be able to put them behind me. Couldn't even eat or drink since I fell into it (Semel 96). Later, s/he confesses that the poems transform her/his own subjectivity: "Without meaning to, I saw my own world decompose into the most basic concepts, and I'm a little girl and a rat too" (97). The act of bearing witness is one of traumatic transfiguration, of becoming the traumatized subject through the act of listening, which suggests that it is not only the victims of a catastrophic event who need 
justice and healing to assimilate their experiences, but the entire world. Nave@infomail confirms the necessity of traumatic transfer and transfiguration when s/he writes, "I think it's important for as many people as possible to get to be the other way around," and sends the poems onto Stash (96).

Ninety years later, in a future world of 2099, a mysterious "Y-mee Prana" bears witness to what we can surmise have been exponential transfers of the poems and the legend onto future generations. Y-mee Prana chronologizes the transference, saying, "Girl \& Rat first appeared on the ancient internet some time towards the end of the first decade or the beginning of the second decade of the century. It was a short cycle of poems, and in one version it included a legend too [...] it's very hard to tell which [versions] are 'authentic' "' (Semel 121). When the novel begins in 2009, Girl-NowGrandmother is the story's only living witness. Through the granddaughter's legend and the subsequent poems "ma[king] their appearance in the digital domain in this century," however, "they gave rise to a huge industry that has been taking on various shapes ever since. For more than nine decades, Girl \& Rat has been told and recorded on every medium imaginable, and you even find its imprint in extraterrestrial human colonies" (122). Y-mee Prana explains: "Girl \& Rat became the visual image most likely to be found on diaries, calendars and PDAs [...] Girl \& Rat stickers are on display [...] Girl \& Rat shrines (123-4). Mickey Mouse turned into "Mickey Rat" (124). As these commercial uptakes of Girl \& Rat suggest, the transferred story becomes sensationalized, while the lived story is too unbearable for the grandmother to utter to her own flesh and blood, her granddaughter. This contrast underscores how witnessing the Nazi Holocaust was 
impossible for survivors; therefore, witnessing must happen through transference of survivor knowledge from one generation to the next.

Father Stanislaw dramatizes traumatic transference when he observes that Girl "hunkers in her niche like a clump of mud, but it is I who wallow" (Semel 171). Girl "hunkers," while Father "wallows." Where Girl begins to become human again in the safety of Father's church, he wallows, an animal movement, taking on her traumatic transformation of "learning to behave like a perfect subterranean creature" in the pit. This transformation takes place as he continues to shelter her: "I hop. I sniff. My whiskers twitch. My ears are upright. I beat my hairless tail against the walls. I am her human rat. I pad our den with leaves - a warm cradle for our young. My teeth keep growing, which is why I must keep gnawing. All this time I go on looking for escape routes, because our very lives depend on them" (200). Father is reenacting the desperation of being imprisoned in the pit with her, attempting to recreate the story in such a way that she can escape the pit and perhaps, evade The Stefan altogether. Father writes, "The more I become Stash, the emptier her memory becomes, and the fuller my own. What was wrought upon her in the dark is branded into me. I carry the burn for her now" (207). “ 'We were wrought in secret,'" he tells Girl (emphasis added, 224). "Against all forgettings, this memory shall prevail," Father promises (231). While the granddaughter, Nave@infomail, Y-mee Prana, Father Stanislaw, and the world may never know the complete story of what happened to the little girl, transferring her experience by way of surrendering oneself to its messy, horrific details brings relief to the victimized, and holds the world accountable to bearing and remembearing that victimization so that it may never happen again. 
In addition to reconfiguring trauma as a transmission across generic forms, time, and space, And the Rat Laughed also speculates about alternatives to psychiatric understandings of trauma as dwelling outside of language. As the Girl \& Rat legend and the anonymous poems spread across the World Wide Web, Rat situates trauma inside of language, linguistic packages for the remembearers to decode. However, psychiatric theories of trauma have normalized trauma as dissociative and therefore, noncommunicative. In The Body Keeps the Score: Brain, Mind, and Body in the Healing of Trauma, Bessel A. van der Kolk, M.D. uses neuroimaging to show how the part of the brain controlling speech functions (Broca's area) goes “offline” when patients with posttraumatic stress disorder experience flashbacks, rendering them unable to put words to their thoughts and feelings (43). From this observation, van der Kolk concludes that "all trauma is preverbal" and pre-cognitive (ibid).

In reaching this conclusion, van der Kolk makes several assumptions: that verbal language is an/the ideal mechanism for expressing traumatic experience, and that words concretize thoughts and feelings; that the speechlessness survivors experience during a flashback is permanent - that is, that every time they experience a flashback of or recall the traumatic event, they will be speechless; and, that these sudden vivid memories of past events, experienced by an individual, are the gold standard for studying the relationship between trauma and language. None of these assumptions hold true in Rat. As the multiple transferences of Girl's story throughout the novel suggest, traumatic experience cannot only be conveyed through verbal language, but it can also be communicated nonverbally, such as when Girl's "soundless body, with its stench of excrement, all its limbs dripping" arrives at Father Stanislaw's church, transmitting to 
him the malnourishment, neglect and abuse she sustained in hiding (Semel 165-66). In caring for Girl, Father surmises the commonality of her experience, wondering, "How many children of pits and of basements, children of cupboards, children of boxes and niches are coming out of their holes now?" (226). The body, while soundless, keeps the score, just as van der Kolk argues_—what feminist political theorist Christine (Cricket) Keating identifies as an engaged silence of witnessing — but it also transmits. The horrors the Nazi Holocaust wreaked on Girl's body and the bodies of other Jewish children are communicated not through the testimony of any single survivor, as And the Rat Laughed expresses Girl's story as a narrative suspended across five genres; across past, present, and future; and, across multiple narrators.

Elaine Scarry's groundbreaking, The Body in Pain: The Making and Unmaking of the World, explains the phenomenon of how pain, when shared, becomes expressible (i.e., verbalized) as others "speak on behalf of" those in pain (6) and as "verbal documents" such as poems or personal injury trial transcripts "record the passage of pain into speech" (9). The disjointed legend Girl relays to her granddaughter, which transform into the anonymous poems discovered and passed along via the internet, and the "Girl \& Rat Myth" that emerges from these compiled acts of bearing witness "record the passage of [Girl's] pain into speech.” This passage into speech is made especially palpable when Nave@infomail describes how the writer of the poems "decomposes the world into the most basic concepts, but presents them the other way around. You'll sense it - the innards pouring out" (Semel 95). Trauma alters how pain is passed along, but it does not stop the transmission from happening. For instance, Girl does not speak when she emerges from the pit; "her mouth is still sealed" (183). But her body reveals the neglect 
and abuse she sustains, prompting Father Stanislaw to bring her story into language by way of his diary, creating a verbal document about sexualized Nazi Holocaust genocide.

Girl herself eventually puts her trauma into words. "The story is between her legs," Girl-As-Grandmother states when she is telling "the legend" to her granddaughter (Semel 37). This statement illustrates van der Kolk's theory that the body carries memories when the mind cannot recall them. But this statement's very linguistic form, as a sentence, also exposes gaps in the logic of "the body keeps the score." Rather than breaking down language, this utterance suggests, trauma relocates language such that its story is a physiological place. Moreover, this utterance reasserts the fundamental interdependency of mind and body that so much of modern Western medicine splits.

Semel's use of the nouns darkness, a pit, potatoes, and the War frame Girl's traumatic experience as dialectical — at once mundane and extraordinary, bounded and infinite - as is evidenced by Girl-As-Grandmother's statement that "Darkness, a pit, potatoes, and then the War was over" is the totality of her story (Semel 61). Darkness, the leading descriptor, signifies the all-encompassing "subcutaneous substance" that even has a taste. Contrary to DSM-5's construction of PTSD as a failure to fight off an event, Girl's utterance of “darkness” characterizes her trauma response as an agential internalization of her surrounds, darkness - what we are conditioned to associate with absence, with other. Pit refers to an earth-body, a physical space beneath the earth's surface, a site of excavation. In one sense, Girl's utterance of “pit” indicates her literal location during the Nazi Holocaust. Pit also refers to the center or core of something, such as a fruit, which suggests that Girl's utterance defines the physical pit as more than a place, but a center. Yet also, pit refers to a physiological place inside the human body: the 
pit of the stomach where nervous energy knots and churns; the pit of the female body, the vagina - a bodily hole that the penis penetrates. Even as Girl does not utter the word "rape," as a medical doctor making a PTSD diagnosis would be inclined to identify as the triggering event, the language she does use insinuates the sexualized violence she sustains. Further, it signifies what a catastrophic event like the Nazi Holocaust means to one survivor may mean something radically different to another-that we should resist the compulsion to generalize Nazi Holocaust historiography. The "center" of trauma for Girl is the potato cellar where the farming family stows her away; The Stefan raping her. These "secret" traumas must be accounted for.

In following darkness and a pit in Girl's list of details, potatoes also function as a linguistic representation of the dialectical nature of traumatic experience. Potatoes represent the mundane and the extraordinary. Potatoes are basic material objects of sustenance, plant tubers, that Girl gnaws on while she is hidden in the pit. A readily available source of vital nutrition that supplements daily scraps the farming family throws down to her, potatoes help Girl to physically survive. Potatoes are a common, affordable staple food around the world, and have been transformed into numerous hybrid forms. In the United States alone, there are over 100 varieties of potatoes available for sale (Potato Association of America). As journalist Michael Pollan explains in The Botany of Desire: A Plant's-Eye View of the World, potatoes have tremendous nutritional and symbolic value in human history, as they have come to our rescue in times of great need. For example, in the late sixteenth century Europeans discovered that the potato "could produce more food on less land than any other crop" (199). Easy to plant as "spuds were simply laid out in a rectangle on the ground" and then covered over "with whatever soil, 
sod, or peat came out of the trench," potatoes provided the Irish with independence and autonomy (200). Potatoes enabled them to "feed themselves off the economic grid ruled by the English... [f]or the Irish had discovered that a diet of potatoes supplemented with cow's milk was nutritionally complete" (ibid). Much as potatoes symbolize the human capacity for adaptation, potatoes in Rat facilitate Girl's survival of the pit.

When read symbolically, Girl's utterance of potatoes also alludes to her psychic adaptation to human violence. Potatoes are a comfort food. Moreover, Christian England regarded potatoes as "mere food" or "animal fuel" in contrast to wheat, which "must be harvested, threshed, milled, mixed, kneaded, shaped, baked" before the "final miracle of transubstantiation [when] the doughy lump of formless matter rises to become bread" (Pollan 203-04). While wheat has come to symbolize “civilization's mastery of raw nature," potatoes have come to symbolize the opposite - a surrender to raw nature"gradually muddying the distinctions between man and beast, even man and root... putting nature back in control of man" (203). In essence, Pollan's study suggests, potatoes animalize us, returning us to our earthly origins. In uttering "potatoes," in selecting them as one of four details that define her Nazi Holocaust experience, Girl rejects the bigotry of "civilization" by way of resituating the human and the animal in relation to one another.

After potatoes, "the War was over" (Semel 61). Translating this last part of the sentence that Girl utters as the totality of her story, we might interpret it as saying that World War II ends after Girl transfers the bottomless, intangible darkness into substance; survives the enclosure of the physical pit and the invasion of her own bodily pit; and, finally, nourishes her body with nutritious potatoes - the very organism with which she is 
forced to keep company. To end her story with the ending of World War II exposes the pervasive assumption that once the War ended, Nazi Holocaust trauma ended. To end her sentence with this detail is to subtly tell her granddaughter she is done trying to remember, trying to speak; that she should ask no more questions. Yet it also implies something more - that the story must continue; that we reader-witnesses must hold out for the full story by reading the many pages to come.

Traumatic experience is often theorized as atemporal and preverbal, beyond the scope of a coherent, chronological narrative of what happened. And the Rat Laughed overrides this premise - to represent trauma as not so much atemporal but intratemporal, between the layers of past, present, and future; to represent trauma as not so much preverbal but intraverbal, literally between the lines. Among writer, language, paper, and reader, experience is remapped — from bodies that endure trauma to a world of infinite bystanders who must witness "the innards pouring out" (Semel 95). The poems that provoke Nave@infomail's utterance here transcribe experience into language decades after the Nazi Holocaust. To say that you, reader, "will sense it," is to say that trauma may exceed, but is certainly transmitted through, language. Sensing, feeling is vital to remembering trauma " $[\mathrm{b}]$ ecause only by reacting will we remember... even if we don't quite know what it is that we're supposed to remember" (98). We might therefore read each poem as if it begins with the word, "remember," even as it does not ever appear in the poem. Remember "I have a big pit outside me/I have a little pit within/The big pit is mine/The little pit is the Stefan's" (100). To remember is to be ever in the middle of what happened, and what might yet happen. In being in the novel's literal middle as Part Three, for example, the poems - lacking any known author or date of original 
publication - transmit "the innards pouring out." We do not know exactly who wrote them, although we can infer that they are written in the voice of Girl, as the granddaughter's classmates understood it when they heard the legend. The point is to read them, and respond.

All of the poems are written in the present verb tense, ever salient in the present moment. While discovered decades later, their present tense is preserved - their "nowness" made palpable, again and again and again, to whoever encounters them. Traumatic experience exceeds the bodies to which events occur, even as the poems are written in the first person. Anyone who hears them becomes a victim. Trauma is not individual, interpersonal, or event-bound, but rather, collective - a thing with substance that sticks to witnesses, that reveals wounding across multiple "who" 's and "how" 's. As the novel's futuristic Y-mee Prana attests, Girl's story is a kind of anonymous multiplicity: "Different versions [of Girl \& Rat] still exist, in various languages, but it's very hard to tell which ones are "authentic" [...] assuming there really was an authentic source to begin with, but it was no use. Even the attempt to determine the language of the original amounted to sheer speculation (Semel 121). The traumatic story resists conventional telling. Yet the impulse to tell, to know, to master, to move on, persists - as if the process of trying to tell is trauma, not a definitive event and the conventionality of "this, then this," and so forth. Girl's personal story is a cultural one that reminds listeners/readers that the alleged privacy of rape entangles all. Even if the survivor forgets, the witnesses do not as "the event itself can't be excised from the memory of the other carriers" (150). In other words, even when survivors try to forget - through treatments, recreational and prescription drugs, and myriad other strategies survivors call upon to help them forget 
and "move on" - and even when they are successful in forgetting, the story has a life larger than any individual, transmitting itself to others (e.g., therapists and family members of survivors). With traumatic transference, "I" becomes "we." To invoke Father Stanislaw's diary again, "I was wrought in secret" becomes "We were wrought in secret" (224).

Perhaps theories that trauma exists entirely outside of time and language may be more appropriate if revised to suggest that time and language can express trauma only when approached out of order, when we are stripped of the impulse to make chronological, coherent history. From an anthropocentric perspective, language is alphanumeric and human-made. Language distinguishes us from other living beings. However from an animal perspective, as rendered by David Abrams in Becoming Animal, language is a vehicle through which we enact our interbeing with the earth. Language is an instrument of connection, relationships - "not to re-present the world around us, but to call ourselves into the vital presence of that world - and into deep and attentive presence with one another" (11). Language then is not human property, but rather "a property of the animate earth itself, an expressive, telluric power in which we, along with the coyotes and the crickets, all participate" (171). To claim that all trauma is pre-verbal, as van der Kolk does, or that the Nazi Holocaust produced no witnesses as Laub claims, is to imply that trauma precludes interbeing-ness and connection, which decontextualizes traumatic experience from human manipulations of power and control from which trauma emerges — whether PTSD is prompted by domestic violence, rape, war, sudden death, or some other human-to-human violence. If "language" is a property of the earth and not exclusively of humans - if language is greater than the sum of what we so often assume 
are its only or its most important parts (words) - then trauma can be spoken, can be witnessed. Language is highly social. If trauma stops outside the confidential doors of the therapist's office, then the world will keep on breaking itself, which supports my claim in Chapter Two that mental healthcare costs more than it cares.

\section{A Conclusion}

What is particularly unique about Nava Semel's And the Rat Laughed is its sustained meditation on the relationship between trauma and language, between what happened and telling what happened. Language breaks down, only to rearticulate itself again, ever attempting to surround, enclose, and narrate the catastrophic — at the same time as the articulation of coherent story is impossible, bringing the speaker into contact, as it does, with events that split psychic and physiological experience, and that remind survivors that others did not survive. Aroused and stirred at pivotal moments in the story, language transfers traumatic experience onto other subjects. Language avoids naming. Yet language is also a mechanism through which we come to know the pit and what happened to the little girl there - a mechanism through which we come to remember. Trauma can be read off of the body; and, the body is a resource for filling hermeneutical gaps in articulating and narrating trauma. Trauma is at once benign and malignantmoldy potatoes and girl's loss of her parents; a pit and vaginal rape; lice and the allknowing sense that you are hated. Language interchanges, substituting words with benign connotations for ones that signify violence.

And the Rat Laughed is an enactment of trauma circumscribed by language, an enactment of interbeing. Trauma and language reveal each other as excesses, thrums, in- 
betweens. Language transfers the excess of traumatic experience onto other subjects, and it is also the thing that interrupts any kind of knowing with certainty. Language is both fantastical and mundane, malignant and benign. Trauma is at once potatoes and malnourishment, pit and vaginal rape, lice and neglect. Language itself does not heal, yet it prompts each character's own journey of working through Nazi Holocaust trauma. Trauma is circumscribed by language, bounded within the material space of the novel, yet also exceeds the text — a liminal space between mind and body, brought about through the unimaginable rapings of a five year-old girl.

And the Rat Laughed's representation of sexualized violence as a mechanism of Nazi genocide, in addition to the novel's sustained meditation on how the Nazi Holocaust continues to entrap Israeli society in acts of survival that circumvent mourning, point up an ongoing need to bear witness to survivor testimonies. Each of the novel's five partsThe Story, The Legend, The Poems, The Dream and The Diary—shape Girl's traumatic story through a composite of witnesses: first-hand testimony that Girl shares with her granddaughter, her granddaughter's (mis)interpretation of that testimony, and configurations of "truth" that past and future generations construct when encountering the (mis)interpreted testimony. As Father Stanislaw speculates in his final diary entry, dated 28 February 1945, "Maybe history is a kind of story, a kind of poem, a collection of legends or dreams that people tell themselves at night. And these stories and legends and poems and dreams embody the truth, in a code that few will want to decipher" (Semel 230). As Stanislaw's entry attests, the generic assemblage that is And the Rat Laughed distinguishes the text as a work of Nazi Holocaust historiography. Thus, readers of the novel have an opportunity as well as an ethical obligation to engage in reading as an act 
of bearing witness - not only to Girl's story and to her granddaughter's (mis)interpretation of that story, but to the process of witnessing itself.

Writing this chapter has been by far the most transformative in my understanding of traumatic testimony. This text does not want to be written about. I am coercing it into critical shape. The very process of writing about And the Rat Laughed directed me, again and again, towards reading between (genres, narrative points of view, space and time) to even come close to knowing what Girl experienced in the pit. At each moment of arrival at a tentative conclusion, another bulged forth, rendering the original incomplete, simplistic, suspect, or even altogether false. Girl's story existed long before the materialization of And the Rat Laughed by spotlighting the obscured experiences of raped girls and women during the Nazi Holocaust. To draw from Ronit Lentin's gendered analysis of The Shoah, translated from Hebrew as catastrophe, Israel and the Daughters of the Shoah: Reoccupying the Territories of Silence, I argue that Girl's story "reoccupies a territory of silence," convoking Jewish girls' and women's experiences of sexualized Nazi Holocaust trauma. As Lentin explains, territories of silence refer to gaps between embodied experience and representation in relation to the Shoah, which result from the banishment of particular events from consciousness in an effort to survive. Reoccupying those silences, Lentin notes, is an act "of mourning the loss of life that was the Shoah, and the inability, or unwillingness, to mourn that very loss by an Israeli society absorbed in acts of survival" (1). Girl's story voices silenced histories of sexualized violence, continuing beyond the novel's materialization as well, into its carriers - reader-witnesses, sexualized violence survivors, and other storytellers who write about what it means to survive the Nazi Holocaust. 
Just as trauma pushes the boundaries of "truth" to encompass a diverse range of witnesses, my writing comes dangerously close to an edge, threatening to spill over with no return and to carry me beyond the limits of what I believe I can make sense of and what I can say. And the Rat Laughed brings me into the subterranean earth, beckoning me to know what I know in the darkness of incomplete thoughts, of conclusions decategorizing themselves at the very moment of trying to articulate them. To write about And the Rat Laughed is to be acted upon by trauma and to become a reader in-between. In the next and final chapter, I describe how the literature classroom can become a site for reading between - a site of collectively bearing witness to trauma. Through a pedagogical case study of teaching Alan Duff's novel and Lee Tamahori's film adaptation of Once Were Warriors, a fictionalized account of how European colonization continues to traumatize Mäori communities in New Zealand, I argue that literary scholars engage in decolonial activism when teaching students to read as an act of bearing witness to survivor testimonies and to the process of witnessing itself. 


\section{CHAPTER V}

\section{CO-WITNESSING TRAUMA: BEARING TRAUMATIC WITNESS IN LITERATURE CLASSROOMS ${ }^{23}$}

As the previous chapters have concluded, conventional trauma discourses, such as The Diagnostic and Statistical Manual of Mental Disorders V(DSM-5) psychiatric taxonomy of mental disorders, the (bio)medicalization of trauma individualizes and pathologizes trauma; while literary and cinematic trauma discourses, such as female encounters with rape represented in Girl with the Dragon Tattoo and And the Rat Laughed, functionalize responses to catastrophe as agential subversions of the racialized, gendered systemic pathologies that produce sexualized violence. Where psychiatry approaches the study of traumatic disorders such as posttraumatic stress disorder (PTSD) through the individual patient, Dragon Tattoo and Rat approach the study of trauma through the interrelated social conditions that enable and shape sexualized trauma. Examining these medical and literary/cinematic methodologies alongside one another elucidates how different methodologies for studying trauma produce different understandings of trauma itself-understandings that are sometimes at odds with one another.

Bridging this conceptual, perceptual gap between the singular patient and corrupt institutions, between healing interpersonal wounds and transforming global injustices,

\footnotetext{
${ }^{23}$ This chapter is an extended version of my previously published article: Johnston, Emily. "Trauma Theory As Activist Pedagogy: Engaging Students As Reader-Witnesses of Colonial Trauma in Once Were Warriors." Special Section on Pedagogy. Spec. issue of Antipodes: A Global Journal of Australian and New Zealand Literature 28.1 (June 2014): 5-17.
} 
this final chapter proposes co-witnessing trauma: a pedagogical approach to traumatic testimony in literature and film through which students bear witness to stories of sexualized violence, and they bear witness to that witnessing, bringing together different experiences of witnessing to collaboratively produce a mutually-valued understanding of trauma. A pedagogy of co-witnessing trauma interrogates rape culture, by way of making explicit students' diverse experiences of witnessing traumatic content in literature. Such pedagogy teaches both textual analysis and critical introspection, opening up space for witnessing how rape culture discourses themselves can often push back against traumatic testimony, in their strategic refusal to understand or believe trauma survivors. Cowitnessing trauma expands students' interactions with texts, and facilitates social justice in the classroom. Contending as they do with what historian Michael S. Roth describes as "the vexed intersection of facts and meaning, events and narratives," traumatic literature and film provide effective portals through which to engage in co-witnessing, by way of listening to stories of what happened, but also to what what happened provokes for students as readers (93). That is, trauma testimonies represent a liminal space between catastrophic events and how those events are remembered, documented, communicated, and otherwise passed down. In listening to traumatized subjects, the reader-witness validates suffering and enacts solidarity. Through the relationship between reader-listener and text-speaker, a form of reciprocity called into being through witnessing, trauma reemerges as a co-creation of fact and meaning.

To investigate how teaching traumatic literature and film can catalyze cowitnessing trauma, this chapter asks: What can bearing witness lead students to understand, or even refuse to understand, about trauma? What can trauma lead students 
to understand about witnessing? What pedagogical approaches do both trauma and witnessing call for that may differ from approaches in other literature classrooms? While this chapter seeks not to arrive at set answers but to articulate the importance of posing such questions in the first place, three preliminary answers have emerged from my internship experience teaching an undergraduate course on sexualized violence in contemporary global literature and film, "Gender in the Humanities: Gender Violence in Global Contexts.”

Firstly, bearing witness can lead students to understand trauma not as narrative chronologies of facts and events, but as testimonies to the excesses produced by such facts and events - such as the feminist vigilantism Lisbeth Salander enacts in Girl with the Dragon Tattoo, and the sensorial hypervigilance the little-girl-who-once-was dramatizes in And the Rat Laughed. However, bearing witness can also lead students to refuse to understand trauma testimonies, particularly when rape culture discourses strategically reject the "truth" of survivor testimonies by way of "they were asking for it" rhetoric. Yet bearing witness to what witnessing provokes for students - even and, perhaps, especially when bearing witness provokes resistance - provides an opportunity for elucidating such entrenched ideologies, and weaving them into discussions of how we shape and how we are shaped by cultural narratives around trauma. Secondly, trauma can lead students to understand that bearing witness is a restorative process that facilitates decolonial social justice. Thirdly, trauma and bearing witness call for pedagogical approaches that augment conventionalized modes of reading and responding to literary texts - approaches that subvert the colonial impulse to discover and conquer, to lay 
claims to particular readings, and to construct and defend arguments about what texts mean.

\section{Reading As Witnessing}

What does it mean to bear witness to textual trauma testimonies, as distinct from oral or "live" testimonies, particularly when those testimonies are fictionalized? Bearing witness to textual trauma testimonies is a process of reading traumatic stories - with an understanding of traumatic story as an attempt to organize and assimilate catastrophic experience. Restructuring memory and cognition, trauma fundamentally restructures narrative away from its conventionalized associations with linearity, chronology, and plot. Trauma produces excess - that is more memories or intrusive reexperiencings in the aftermath of traumatic events (e.g., flashbacks, nightmares) than are desired, and an inability to moderate these memories or reexperiences. Traumatic excess, in DSM-5 terms, signals traumatic arousal, which I defined in chapter two as a metaphorical alarm clock going off in the brain; an anxious awakening. Overwhelmed by horrific external stimuli, traumatized subjects may not process what is happening while it is happening. Following such logic, trauma is aftermath, a perpetual conflict between doubting and denying, uncovering and testifying to, what happened. It is to this conflict that studentreaders can bear traumatic witness.

In her landmark psychiatric study linking interpersonal traumas of relationship abuse and structural traumas of war, Trauma and Recovery: The Aftermath of ViolenceFrom Domestic Abuse to Political Terror, traumatologist Judith Herman, M.D. posits that trauma suspends and even makes narrative obsolete, "surfac[ing] not as a verbal narrative 
but as a symptom" that at once signals "the existence of an unspeakable secret and deflect[s] attention from it" (1). In Dr. Herman's assessment, the symptoms of posttraumatic stress disorder are manifestations of internalized struggle, between survivors' inability to recall what happened, and their profound need to assimilate the experience into personal and collective consciousness. Catastrophic events can thereby set in motion a chronic conflict between denial and telling, rendering cohesive narrative accounts impossible, at the same time as they require someone to listen to the storysomeone to bear witness to the event(s).

Examining the effects of traumatic memory and narrative subversion on witnessing, psychoanalyst Dori Laub, M.D. characterizes traumatic witnessing as a "ceaseless struggle" (61) between survivor and witness to uncover "buried truth" (63). In interviewing Holocaust survivors, Laub found that living through the genocidal horror of the Holocaust obliterated the possibility of bearing witness - a process he defines as witnessing "the truth of what happens during an event" (65). Describing his own Holocaust memories as "discrete islands [...] the remembrances of another child, removed, yet connected to me," Laub communicates the impossibility of assimilating what transpired - that is, of witnessing himself as the bearer of what transpired (66). Being inside of the "coercively totalitarian and dehumanizing framework" of the Holocaust, he writes, "made unthinkable the very notion that a witness could exist" (ibid). No one, neither Nazis nor prisoners could observe the Holocaust from the outside; there was no outside. Indeed, Laub concludes, "one might say that there was, thus, historically no witness to the Holocaust" (ibid). Without witnesses, there is no history. Without history, there is no validation of Holocaust experience. As we saw in the 
previous chapter, witnessing must come from the outside, rendering traumatic history a kind of co-created truth between survivor and witness. In Nava Semel's And the Rat Laughed, Girl-As-Grandmother cannot provide her granddaughter with an eyewitness account of the Holocaust, and her story only coheres through multiple acts of transference onto Father Stanislaw, who intuits Girl's trauma when she arrives at his church; the granddaughter, a second generation survivor fixated on recording her grandmother's Holocaust experience; and, future generations that encounter fragments of Girl's story decades after the Holocaust ended.

While genocidal traumas like the Nazi Holocaust render insider witnessing impossible, Laub identifies two additional "levels" of traumatic witnessing that enact a collaboration between survivor (the traumatized subject) and listener (the figure of the witness), an implicit togetherness invoked by acts of telling and listening. The second level of witnessing Laub identifies, "being a witness to the testimonies of others," entails listening to survivors' accounts, "reliving and reexperiencing" the event without being "submerged" by it (61-62). Laub's third level of witnessing, "being a witness to the process of witnessing itself," involves examining the relationship unfolding between survivor and listener as they try to account for the event (61). By bearing witness to witnessing, a witness recognizes that it is not a survivor's recollection of what happened that validates history, but rather, "the sense that there is a truth that we are both trying to reach;" "[t]he horror is, indeed, compelling not only in its reality but even more so, in its flagrant distortion and subversion of reality" (62). The very subversion of narrative truth, Laub suggests, is evidence of trauma. If students deny the Nazi Holocaust and refuse to position themselves as co-witnesses, that very refusal is itself evidence of bearing witness 
to the process of witnessing, of responding to reading traumatic testimonies. When denial and refusal are treated as artifacts of bearing traumatic witness, as two of many possible responses along a spectrum of possible experiences when reading traumatic testimonies, productive space opens up for discussing that refusal as an inherited cultural denial of many survivor testimonies - as a powerful ideological system that shapes our understandings of catastrophe.

Following Laub and Herman, if traumatic events effectively erase themselves while they are happening, then listening to survivors' testimonies and reflecting on what that listening does may be the only means of bearing traumatic witness. While reading about traumatic experience is certainly a more removed kind of listening than hearing a testimony first-hand, students can enact Laub's second- and third-level witnessing by listening to the text. Collectively, students can synthesize what they are hearing, and then examine what listening provokes for them. Reading traumatic literature facilitates cowitnessing trauma as students encounter characters' traumatic experiences as well as their own traumatic experiences, disseminating their experiences of witnessing in a classroom setting.

\section{Listening to the Text}

In literature classrooms, listening to the text is a mode of critical reading that can teach students how to read and to hear as acts of bearing traumatic witness, in service of global social justice. In listening to the text, students first establish consensus on what they "hear" a text saying before critiquing or evaluating it. Developed from Krista Ratcliffe's concept of rhetorical listening explicated in Rhetorical Listening: 
Identification, Gender, Whiteness - "a stance of openness that a person may choose to assume in relation to any person, text, or culture"-listening to the text encourages students to develop awareness of their points of resistance and engagement, but to do so with a disposition of curiosity rather than of judgment (17). Cultivating dispositions of curiosity provides students with opportunities to actively listen to their prior knowledges and experiences, and to adapt those knowledges and experiences to make sense of new, unfamiliar, or otherwise challenging material.

During my teaching internship, I tested the efficacy of listening to the text by starting each class period with journaling or small group discussions in which students responded to questions that conjoined close-reading and personal reflection. While I modified these questions from one reading to the next in relation to specific themes and concepts explored in those texts, the general sequence of questions was as follows: What is the text telling you about trauma? How does the text align with what you already know, feel, believe, think, and sense about trauma? How does the text extend, challenge, or complicate what you already know, feel, believe, think, and sense about trauma? Asking students to first synthesize what they read in relation to traumas represented in assigned readings - to listen to rather than impose interpretations of the material, as a precursor to integrating that material into their own personal histories and subjectivities — cultivated a collective experience of listening to the text. In beginning with these three reflective questions instead of, for example, evaluative ones, the class structure emphasized the value of listening before critiquing, ingraining this listening practice as a vital first step in confronting injustices represented in the texts we read. 
Journaling about the three reflective questions in relation to "Taking Risks: Implementing Grassroots Community Accountability Strategies" by Communities Against Rape and Abuse (CARA) led one student, who I shall refer to as "Adam," to experience fresh insights into his own privilege as a white male. I assigned this particular reading as a framework for understanding sexualized violence against women as institutionalized misogyny and institutionalized racism in the literary texts we read, as CARA's article explicates how sexualized violence is a systemic issue, inflected by racism and sexism, and which must be responded to not just by individuals directly involved in a particular incident, or by legal interventions alone, but with "communitybased strategies for safety, support, and accountability" that "vary widely on the basis of values, politics, cultures, and attitudes," which differ from one community to the next (250). Adam writes,

To be honest I really do not have many encounters with law enforcement, so when this journal was assigned I was stumped. After thinking about it for a few minutes one encounter came to mind that was relevant. I was in high school when five of my friends packed my car and decided to take a drive thru [sic] the city. Living in the outskirts of Chicago, my friends and I would often times go cruizing [sic]. During this occasion we were driving thru [sic] a bad neighborhood and we got pulled over by the Chicago police. I have tinted windows so it is really hard to see who sits inside, my full car threatened the police officer. He called to me and told me to get outside the vehicle. After I got out he realized that it was "only" five white kids [and] he told me that we shouldn't be in this area of the city and that we should leave. Not [until] later did I realize that my luck was caused by 
racism. The police officer saw that we were all white therefore no longer suspicious. My gut tells me that if we were black or hispanic we'd all get searched and harassed by the cops. The police officers in these neighborhoods go out of their way to target minorities. I'm sure life would be much more difficult if you were presumed guilty until proven innocent. Americans need to change the way they profile minorities and abolish this ideology.

As this journal entry suggests, Adam's racialized, gendered privilege creates an obstacle to bearing witness. While Adam looks at and listens to his personal experience of driving through "a bad neighborhood" in Chicago with his friends, and he attempts to empathize with what it might be like to be "presumed guilty until proven innocent," Adam does not check and question his own privilege. Rather, he defaults into a generic directive for Americans "to change the way they profile minorities"-a statement that ultimately reveals Adam's racist assumption that "American" equals "majority.” As Mariana Ortega explains, such loving, knowing arrogance is arrogance, because "checking and questioning [which Adam does not do] are necessary if the perceiver is to avoid making up a reality that reinforces his or her worldview" (61). Adam wants to perceive with love, but ultimately, this intention effectively reinforces his own desire to set himself apart from the phenomenon of racial profiling.

That said, through the act of reading CARA's article and writing about a related personal experience, Adam lays the foundation for bearing witness to his own ignorance. His "I was stumped" statement at the beginning of his journal entry underscores a particular theme to which I asked students to bear witness in this reading: how institutionalized violence is racialized. As Adam writes, he implicitly attributes his lack, 
his "stumped"-ness, to his social privilege as a white male. He recognizes that while his privilege is not something he asked for - that is, he and his friends did not ask to be let go after the officer pulled them over and discovered they were white- - his privilege perpetuates injustice by the very institutions that profess to protect the public. Through connecting the reading's theme of systemic violence to his direct encounter with law enforcement, Adam has an opportunity to recognize his own privilege. Recognition plants a seed for envisioning a different future.

Another student, who I shall refer to as "Amy," experienced the chronic conflict between denial and telling that trauma induces, as a result of journaling about the three reflective questions in relation to a series of chapters on sexual harassment in workplaces and schools in Gender Violence: Interdisciplinary Perspectives. I assigned these articles to facilitate understanding of how, in the literary texts we read, institutions shore up their privileged social status and power by way of rendering invisible women's experiences of sexualized discrimination. As Senior Research Scientist at Wellesley Centers for Women Nan Stein, Ed.D. indicates in the chapter, "Locating a Secret Problem: Sexual Violence in Elementary and Secondary Schools," sexual harassment in K-12 school settings is on the rise, "occurring at younger and younger ages" and becoming increasingly violent (323). Stein goes onto explain that sexual violence in schools "often gets named as something else in an effort to cast it as benign (e.g., the use of the term 'bullying'); [...] is not reported to law-enforcement or school officials; [and] when it is surveyed, it is not disaggregated from incidents of physical violence" (323). In response to Stein's analysis of the invisibility of sexual violence in schools, Amy narrates her own experience of assault: 
My story of sexual harassment is hard to fully explain. Last February I was sexually assaulted while at a party. I was drinking, which is how I lost the motor function to get away. It wasn't violent, I don't think. I remember everything before and after. But that's not what this is about. I didn't pursue charges because in all honesty, he doesn't deserve that, and it will ultimately make things harder for me. After it happened (I was living in a dorm) a friend who knew what happened talked to her R.A. [Resident Advisor], not including specifics. This R.A. figured it out, and told her boss, the R.H.C. [Residence Hall Coordinator]. At the time I was applying to be an R.A., so did not want this situation held against me - so I ignored the emails from [titles of organizations omitted for confidentiality and anonymity purposes]. I was fine, I still am, and haven't pursued counseling. I ended up getting an R.A. position. The real sexual harassment didn't start for me until after the fact. I'm supposed to be the strong role model in so many ways, but I'm actually a hypocrite. I seek counseling for so many people but myself. I honestly don't want it. Is it so wrong to think that that would be blowing it out of proportion? But don't think I'm unhealthy. I got a rape kit done with the nurse and I'm cleared. I guess the sucky thing is that him [sic] and I are both seen as leaders of the [title of organization omitted for confidentiality and anonymity purposes]. It's really my family, and it sucks that such a thing had to happen in that environment. Not only that, but I'm not going to leave/quit over this. That's not fair to me. But I do have to not only see him but work with him everyday. He's not a bad person in the least bit. Which leads to this weird doubt in my head that I can't explain. He knows what he did was 
wrong, but we go about it like we're friends. Other people in the [organization] heard about it [...] and look down on me for it. I guess that's the thing I look at as sexual harassment - not what happened, but how people treat me afterwards. Things won't ever be the same, but a strange part of me is happy that it happened — because now I can help others with the same issues. (emphasis added) In the act of writing her story of sexual assault, moved to do so by reading survivor testimonies in Stein's and other Gender Violence chapters on sexual harassment, Amy experiences the struggle to "fully explain" what happens during a traumatic event. She experiences what Laub describes and Semel's And the Rat Laughed dramatizes, in relation to the Holocaust, as the impossibility of bearing witness to oneself within the event, although she recalls "everything before and after." Evidenced by qualifiers like "I don't think" when she says the assault "wasn't violent," and by her rush to excuse the perpetrator as "not a bad person in the least bit"-someone who "doesn't deserve" charges brought against him and who, in fact, she considers (or at least treats like) a friend - Amy bears witness to the "weird doubt in [her] head that [she] can't explain." Through writing, she actually answers her own implied question of why she experiences doubt: the social context in which the assault occurred normalizes male violence to such a degree, Amy fears "this situation [being] held against" her in her application for a Resident Advisor position on campus. She almost sounds surprised that University Housing Services hired her. Amy notes that some of her work colleagues even turned against her, suggesting that she believes, on some level, she is responsible for the assault.

Traumatic events can induce disorientation, as Amy's testimony here evidences. Yet moreover, Amy's disorientation evidences the ways in which bearing witness to 
textual trauma testimonies such as those found in Stein's article on sexual harassment in K-12 schools, by way of the three reflective questions, creates space for student-readers to bear witness to their own traumatic experiences, the meanings those experiences have for survivors, and the intersections of students' experiences with those represented in course readings, thereby facilitating the creation of a written artifact (a journal entry) that bears witness to the process of witnessing itself. In writing about acquaintance assault, Amy voices the internalized doubt and self-blame that plague many victims of sexualized violence, and that parallels the internalized shame characters experience in the novels our class read-for example, Alan Duff's Once Were Warriors, which I will discuss in relation to a pedagogy of co-witnessing later in this chapter.

Ultimately, bearing witness to the institutionalized erasure of K-12 girls' assault experiences - specifically, the rendering of those experiences as "benign" by categorizing them as "bullying"-leads Amy to revisit her own assault story. Moreover, bearing witness enables her to reach an understanding of the trauma as not the event itself, but “everything before and after,” especially its aftermath (i.e., her peers’ scorn).

Recognition, while tentative and unsure, plants a seed for understanding sexual assault as a socially reinforced trauma. While Amy's language verges on rationalizing her choice to not seek support, and on vindicating the perpetrator, in the act of exposing her story to paper, of being a witness to herself before and after the event itself, Amy breaks silence, connecting with that "strange part" of herself that feels glad she experienced assault so that she can help other students who experience it as well.

Other student journal entries illustrate similar outcomes to those of Adam's and Amy's writings: that listening to the text, by way of responding to the three reflective 
questions in relation to course readings, facilitates witnessing. Witnessing, in turn, facilitates change in the form of reenvisioning personal experience and utilizing that experience in the service of social justice. After reading Melissa S. Fry's article on the long-time legal sanctioning of rape in the United States, "Becoming Victims, Becoming Citizens: A Brief History of Gender-Motivated Violence in U.S. Law," a student identifies the collective nature of so-called interpersonal trauma, observing that "each individual rape is the violence of society being used upon a single, individual woman." Comparing the experience of reading and later watching rape scenes in Girl with the Dragon Tattoo, another student identifies the sensory experience of bearing traumatic witness, remarking that "[a]lthough reading about [Lisbeth's rape] had made me uncomfortable, actually seeing it [when we watched the film adaptation in class] brought me many emotions that had given me a scratchy neck." In relation to the same topic of comparing and contrasting responses to the Dragon Tattoo novel and film adaptation, yet another student articulates the ways in which bearing traumatic witness summons us to action, writing, "I can't ignore these scenes [...] and I need to just... try and process what they were for Lisbeth and what it means for women and what it means to me." As this statement suggests, the student's processing of rape trauma begins with bearing witness to an individual rape survivor (i.e., protagonist Lisbeth Salander), which in this student's mind, necessitates witnessing what gender itself means for her and other women. Trying to balance this introspective work with her rage at the misogynistic comments Advokat Bjurman makes while raping Lisbeth, this student stumbles in the very act of writing: "These words are just so... infuriating and foolish and gross... I wish I had the right word." Ultimately, the student concludes that Lisbeth's retaliation, recording Bjurman in 
the act of raping her a second time rather than seeking police intervention, "says so much to me about the world - that women are to be used, and even at times we must use ourselves to get ahead." Lisbeth's story prompts recognition.

As the above student journal excerpts illustrate, through listening to the text, students can come to recognize how their perceptions of trauma may differ depending upon their own racialized, gendered, classed, sexualized subject positions. Through such recognition, students can also come to recognize how transforming trauma requires a wide range of interventions, depending on the specific conditions and needs of any given traumatized community. Informed by Laub's claim that traumatic "truth" or validation emerges through interaction — through witnessing —listening to the text can help students inquire into their own reactions and responses to texts not just as testaments of personal opinion or worldview, but of ingrained ideologies that surpass any singular subject. Asking students to identify how a text simultaneously aligns with and challenges their prior knowledges and experiences can help them investigate their reactions as mediated by privilege. Such investigation can ultimately prompt students to envision a more just world, as well as their own responsibilities and rights with respect to creating such a world.

Listening to the text encourages investigation, and subverts the colonial complex of mastery: a sense of entitlement to control and exert superiority over the strange, foreign, non-normative "other." As readers dwell on interstices between what is narrated and their own stories, they bear witness to how one reveals the other-especially when students feel estranged from texts and/or their own traumatic stories. Grounding our reading practices in investigation works to resist the closure that colonialism might have 
us believe in order to perpetuate its own imperialist control: that the days of colonial omnipotence are over, given way to shared governance, and that the poverty pervading many indigenous communities indicates individual dysfunctions and not systemic racism (e.g., the colonial belief that violence and addiction are intrinsic to indigenous groups).

The novel Once Were Warriors facilitates investigative reading as it suspends our very notions of how to categorize and classify literature. In assuming its own superiority, a colonial complex of mastery shapes the practice of categorizing and classifying textsmuch like $D S M-5$ shores up its own rightness in categorizing and classifying so-called mental disorders. Categorizing texts can create distance between reader-listener and textspeaker because it implies mastery — that ultimately, readers can know what a given text $i s$, and classify that text according to particular genre conventions or stylistic features. Once Were Warriors resists categorization, fitting into multiple genres such as fiction, faction, nonfiction; action, drama, crime, even thriller; and, sub-genres of philosophical, political, and urban texts. Teaching Once Were Warriors and critical studies on trauma alongside one another is far more complex than simply "teaching context"-historical, political, cultural. It involves deconstructing conventional modes of engaging with texts (e.g., categorizing texts according to genre), inviting students to practice not knowing with certainty, to resist closure. Dwelling in uncertainty and resistance are prerequisites for restoring global social justice, as they encourage active listening, not just to what is being said, but also to how our own prior knowledges and experiences shape what we are willing and able to hear. Active listening invites us to start in actual lived experience, the words on the page, rather than in theories or external frameworks for understanding literature, and to build understanding of lived experience by suspending our own 
assumptions about what it means or how it should be narrated. In short, reading Once Were Warriors as witnesses can dismantle colonial logics.

Traumatic literature courses embody the paradox of trauma - what Judith Herman, M.D. calls "the central dialectic of psychological trauma," or "the conflict between the will to deny horrible events and the will to proclaim them aloud" (1). Designing a course around trauma's "central dialectic" can facilitate students' understanding of how traumatic experience fractures language (e.g., traumatic arousal produces excess that overwhelms the psyche and our linguistic capabilities). It can also invite modes of inquiry and critical approaches to texts that produce a continuum of meaning along a spectrum of dialectical themes. For example, one might frame a course on bearing traumatic witness in literature and film around contrastive literary themes and symptoms of post-traumatic stress disorder: denial and rumination; terror and safety; isolation and community; captivity and sovereignty. How does terror, for instance, violate our basic human right to safety? How does terror motivate change that can free indigenous peoples from colonial rule? How does procuring safety, in turn, become not only an act of survival, but also of resistance to genocidal injustice? How can safety become a debilitating comfort that obscures truth? These are just a few of the questions that a course designed around trauma dialectics can prompt, inviting students to read in the direction of bearing witness to the "ceaseless struggle" of trauma.

\section{Once Were Warriors: A Context for Understanding}

I invoke Alan Duff's bestselling novel, and Lee Tamahori's film adaptation of, Once Were Warriors as traumatic testimonies to poverty and violence afflicting Mäori 
communities following European colonization of New Zealand. A critique of coloniality, as informed by reading Once Were Warriors alongside theoretical work in trauma, serves several important pedagogical functions: (a) it challenges victim-blaming, the colonial glorification of individualism and personal responsibility in domestic violence situations (e.g., trauma intervenes in the common question of, Why she doesn't just leave?); (b) trauma intervenes in the possible secondary wounding that can occur when students encounter the brutal dysfunction in a novel like Once Were Warriors; and (c) it encourages students to recognize dysfunction not as a self-generated problem, but as a symptom of colonial trauma.

As I will show, the chronic traumas of domestic abuse, rape, and material depravity that characters experience in the novel and film call upon students to bear witness to the ways in which colonization oppresses from both the outside (e.g., by way of the British legal system instituted in New Zealand) and inside (e.g., by way of internalized oppression, teaching indigenous peoples to oppress themselves). A fictionalized account of dispossession plaguing Mäori communities in Aotearoa New Zealand, ${ }^{24}$ Once Were Warriors frames this condition as trauma produced by colonization. Like all colonized indigenous groups, New Zealand's Mäori population experiences ongoing cultural genocide resulting from "a history of subjection to totalitarian control over a prolonged period" (Herman 121). In other words, settler colonialism "inflict[s] conditions of life calculated to bring about physical destruction of

\footnotetext{
${ }^{24}$ Aotearoa, commonly translated into English as "land of the long white cloud," is the original Mäori word for the land now known as New Zealand, named by Dutch explorer Abel Tasman, and English explorer Captain James Cook. Many New Zealanders now refer to New Zealand as Aotearoa New Zealand in recognition of the country's so-called bicultural identity, Mäori and European. In April 2013, the New Zealand Geographic Board sought public opinion on officially changing the country's name from New Zealand to Aotearoa New Zealand ("Aotearoa"). This chapter uses Aotearoa, New Zealand and Aotearoa New Zealand interchangeably to reflect the country's diversity of nomenclature.
} 
the group in whole or in part," such as inaccessible and/or inadequate water, food, clothing, shelter, healthcare, and other basic survival needs ("Genocide"). In addition to being traumas in and of themselves, these conditions signify traces of colonialism in contemporary Mäori communities. Moreover, they contextualize the insidious psychic, spiritual rage among Mäori communities who continue to struggle for survival.

The stream-of-consciousness narration in the novel, and the disorienting imagery in the film, makes Once Were Warriors particularly conducive to bearing traumatic witness, in and beyond classroom settings. Once Were Warriors narrates trauma at the intersections of history and contemporality, representing alcoholism, gang violence, rape, and suicide as products of oppressive colonial institutions which cannot be examined outside of New Zealand's history of subjecting Mäori tribes to European rule. As the back cover states, Duff's novel provides “a harrowing vision of his country's indigenous people two hundred years after the English conquest." Set in the fictional Pine Block, a Mäori state-housing project on the outskirts of Auckland, New Zealand's metropolis, Once Were Warriors depicts the "harrowing vision" of one Mäori community on the verge of extinction. In portraying colonization's simultaneous past and present presence, Once Were Warriors offers readers an opportunity to bear traumatic witness to Pine Block as a traumatized postcolonial community, and to the ideologies that shape our reactions to the devastation of Pine Block.

The three routine questions that conjoined close-reading and personal reflection, analyzed in the previous section of this chapter as a pedagogical method for listening to the text, revealed that the rampant poverty represented in Once Were Warriors can (and did) provoke strong reactions of pity and disgust among students in "Gender in the 
Humanities: Gender Violence in Global Contexts.” The Heke family at the center of Once Were Warriors experiences the loss of not only ancestral lands and spaces, but also of tribal connection. Pine Block offers scraps of culture. Car wrecks become playgrounds for children - "No gardens here. Not trees, nor plant arrangement, not nothing" (Duff 7). The playground of abandoned, wrecked cars - "rusty jagged metal edges that add to their infected, half the time pus-oozing wounds"-wound and prevent healing (Duff 6). In the film adaptation, the Heke children play in the dirt that is their state-housing project playground while their eldest sister (Grace Heke) tells them of taniwh $a^{25}$ mythology. Physical space materializes neglect, abandonment, and a failure of careparticularly in relation to Mäori traditions. Homes are palpably temporary, provisional, disconnected. Homes are holding cells. As protagonist and mother Beth Heke notes, homes are bookless_- "not just her own house but every house she'd ever been in [...] like a sense of loss, almost grief [... because Mäoris] “didn’t have a written language before the white man arrived [...] because a bookless society didn't stand a show in this modern world, not a damn show" (4). Without books, Beth concludes, the Mäori of Pine Block cannot participate in the dominant pakeha ${ }^{26}$ world of industry, capitalism, and global communications. A phenomenon resulting from European colonizers instituting their own forms of schooling, stripping away the oral tradition of Mäori culture, booklessness signifies how colonization has rendered Mäoris dependent on European institutions, at the same time as it continues to authorize Mäori cultural genocide. Beth's despair suggests that without books, Pine Block will remain impoverished, dependent on government welfare. Listening to the text in these moments of describing the

\footnotetext{
${ }^{25}$ In Mäori mythology, taniwha are supernatural water- and cave-dwelling creatures that can both protect and terrorize tribes (Keane).

${ }^{26}$ Pakeha is the commonly-used Mäori word for non-native New Zealanders.
} 
environment of Pine Block challenges students to understand poverty in the larger context of colonial oppression, to approach their reactions of disgust or pity to Pine Block as stemming, in part, from the privileged notion that colonization was amicable, that indigenous groups are now free of European governance, and that struggles they face today are of their own making. Duff's use of booklessness as a symbol of colonial oppression allows readers to glimpse the insides of Pine Block homes, while also providing a conduit to understanding literacy as a master's tool that segregates, isolates, and denies access.

Beyond booklessness, Beth Heke also takes notice of the decrepit state of her neighbors' yards, the narration calling upon readers to bear witness with Beth to the slow death of Mäori peoples and cultures: "[O]ne house had an old wringer washing machine stuck right outside the front door [...] And the grass — sposed [sic] to be the bitch's front lawn - grown halfway up its fat, chipped girth, weeds sticking out its belly, and a slimy moss formed all over the rubber wringer rollers like a disease" (Duff 6). Beth perceives the weedy, slimy, abandoned machine as a kind of colonial artifact—an object of what Beth calls "pridelessness" brought on by the genocide of indigeneity (ibid). Like the washing machine, Mäori peoples exist today as shadows of their former selves. Neglected, what is supposed to be a site of growth and activity, a space of welcome, a first impression — a front lawn, a human being, a culture — is decaying. The battered Pine Block neighborhood is a traumatized subject through which readers can imaginatively sense the trauma of colonial dispossession.

The Heke family is also in a state of loss and decay. Forties of beer crowd the kitchen table. Parents (Beth and Jake Heke) party with their friends while their children 
lie awake upstairs, waiting vigilantly for their father's rage to shatter the spell of drunken merriment. As if on cue, the party goes from love song to bloodbath; from Beth and Jake serenading each other to Jake punching, pummeling, and breaking glass against Beth's head, then raping her, after she refuses to cook eggs for his mate (Uncle Bully). In the film, rape is accompanied by the haunting sound of whirring, which becomes increasingly loud and drowns out voices and screams. The scene concludes with an image of a dog scavenging for food scraps in the trash.

As this staging of the scene suggests, domestic abuse is a terror that at once suppresses and surpasses the individual, the human. The next morning, their daughter Grace finds "a pool of [Beth's] blood on the floor at the table where she must've sat after he'd finished with her" (Duff 22). Home itself is traumatized, a space of both revelation and brokenness, "The whole scene bathed in sunlight pouring in through the black cloth blinds on permanent B for broken [. . .] the floorboards sticky with partly dried beer, and cigarette butts everywhere" (23). Like Beth's battered body, the Heke home is in a state of traumatic excess - desperately needing care, yet caving in on itself. Encountering these images in the novel and film can prompt students to experience the devastation of alcoholism and domestic violence not as indicators of a slothful, barbaric people as colonial logics would have us believe, but of the very intimate, interpersonalized violence of those logics. The government subsidizes ramshackle housing, and while housing provides temporary material stability for the people of Pine Block, this stability offers no sustenance. If anything, so-called "stability" functions as yet another smokescreen over deeper issues of internalized racism that erode away at Mäori culture, turning Mäori people against one another. Moreover, the falsity of "stability" underscores how domestic 
violence intersects with internalized racism, rendering intimate partner abuse as fundamentally social, cultural, collective, political.

Discovering Beth's battered face the next morning, social worker Mr. Bennett remands the Heke's teenage son (Boogie) into social welfare custody. When Beth catches a glimpse of her battered face in the mirror - "if you could call it a face [...] beaten to a barely recognisable pulp [.. . the right eye puffed shut, nose broken—again—lower lip swollen with a deep cut [ . . ] bruises all over"-she begins to identify how domestic violence perpetuates isolation among her people and family, much as my student Amy begins to identify how her assault experience instilled and then exacerbated a sense of disconnection from her peers (Duff 32). As Beth notes earlier, in relation to colonial violence internalized and then perpetrated against Mäori people, isolation is externally imposed: "the good old government, paying em [Mäori people] to do crimes against each other and society" (9). On this very morning, Beth was supposed to appear in court with her son, Mark, charged with shoplifting. Yet she knew her beaten face would betray her - that the judge would see her as an abused woman and render both her home and her parenting unfit.

Beth's act of facing herself in the mirror, ironically, generates self-effacement in a way that she believes will protect her family — an experience akin to the one that my student "Amy" narrated earlier. Just as Beth tries to cover up Jake's abuse in the name of protecting Boogie, asking his sister, Grace, to accompany Boogie to court instead of her, Amy's testimony also validates why she (Beth, Amy) did not seek to punish him (Jake, anonymous perpetrator), and why she feels the abuse happened "after" the assault in how she was treated by others. As both Beth's and Amy's self-effacements suggest, we cannot 
easily or swiftly criticize someone for not seeking justice. Beth's confrontation with her battered face catches her, and us readers alongside her, in the dialectic of trauma between denial, not going to court and masking the truth of her situation, and telling, going to court, and exposing the abuse. Either way, Beth risks losing Mark, just as Amy risks losing friends. And Beth is right: when she fails to show in court, the magistrate says, "I have no choice but to declare [Mark, a.k.a. "Boogie"] a ward of the state [. . .] Asking for the next case. Just like that" (29). The fact that Beth parties the night before Mark's court hearing, and that she continues to stay with Jake after multiple beatings, could lead readers to conclude that Beth is "damaged goods" and that she personally created and chose her life circumstances and, therefore, must change them herself.

However, turning to trauma theory complicates the extent of Beth's agency here and can circumvent the brand of loving, knowing arrogance that student Adam expresses when reflecting upon his encounter, as a white male, with Chicago Police. Institutionalizing Mark (Boogie) is part and parcel of the genocidal mechanism of colonization that has severed many Mäori kinship ties—especially those of children to their families - to the land, and to resources that might allow Mäori youth to unlearn the vicious cycle of poverty, addiction, and violence. Produced by postcolonial oppression and the feelings of hopelessness it instills, Mark's criminal behavior is treated in isolation, through a legal system designed by the original oppressor: the British Crown. Prompted by Mark's legal issues, in the aftermath of this most-recent beating from Jake, Beth begins to reflect on the enduring rifts between Mäori and Pakeha that continue to subordinate Mäori to colonial power. As Beth faces her beaten face, she also confronts racialized stereotypes of Mäori as inherently inferior and in need of taming 
through the colonizer's religion, education, and political control—stereotypes that associate alcoholism and violence with the "passion" of Mäori rather than the coercive genocidal regime of colonization (Duff 37). Beth figures it "must be something in the Mäori make-up makes us wilder, more inclined to breaking the law [...] We got passion, us Mäoris" (37). While Beth appears to succumb to the logic of stereotypes here, she then goes on to link Mäori stereotypes to the fallacy of biculturalism in New Zealand. Beth observes that Europeans are "strangers to most Mäori [Beth] know[s]. May as well be from another country, the contact the two races have" (37). The structure of the narrative - moving from Beth's battered face, to Mark's displacement, to Beth's reflection on the deep rift between Mäori and Pakeha—dramatizes Beth's powerlessness as a product of institutionalized racism. The very system that keeps Beth alive, yet impoverished imprisons the Hekes in Jake's violence, and places their son just out of reach in social welfare custody, rendering him a "stranger."

As Beth comes to recognize this imbalance of power and its effects of her family, she also comes to understand that the fracturing of Mäori culture brought on by European colonization is the root cause of this power imbalance. Beth's process of recognition, linking the traumatic intimacy of her broken marriage and family with the globality of genocide brought on by settler colonialism, parallels the kinds of local/global connections student-readers might make in a co-witnessing trauma classroom. Just a few pages after Beth's reflection on the vast distance between Mäori and Pakeha, she reconceptualizes Mäori "passion" as a colonial distortion of Mäori warrior identity used to render the battle-ready Mäori defenseless against colonization. Mäori scholar Brendan Hokowhitu explicates this manipulation of warrior as a colonial move used to justify taming Mäori 
men, as colonizers tamed and conquered land, for European benefits like manual labor and sports' spectacle. As Beth explains, the identity marker "warrior" went from signifying tribal pride to signifying "savage" under colonial rule. "Warrior," that is, became a tool for teaching the Mäori to colonize themselves - to internalize and enact ruthless violence against self and family.

And we used to war all the time, us Mäoris. Against each other. True. It's true, honest to God, audience. Hated each other. Tribe against tribe. Savages. We were savages. But warriors, eh. It's very important to remember that. Warriors. Because, you see, it was what we lost when you, the white audience out there, defeated us. Conquered us. Took our land, our mana, left us with nothing. But the warriors thing got handed down, see. Well, sort of handed down; in a mixed-up sense it did [...] We- or our men, anyway — are clinging onto this toughness thing, like it's all we got, while the rest of the world's leaving us behind. (Duff $41-2)$

In linking Jake's domestic battery with colonial manipulations of "warrior," Beth connects her personal trauma of enduring domestic violence with the collective trauma of colonization - trauma perpetuated through the very welfare system that removes Mark from Beth and that has removed, and continues to remove, many Mäori from their homeplaces. The stream of consciousness narration of Beth's reflections here allows readers to link collective and interpersonal traumas alongside Beth — to reach this insight through bearing witness to her embodied experience.

Despite Beth's increasing clarity about her lot in Pine Block, the space between what is and what might be remains vast. The life Beth believes she wants closely 
resembles the one she resentfully describes at the beginning of the novel: a white lifeone she believes can rescue her from poverty, addiction, and violence. Beth begins to express hope as she feels her life becoming increasingly "normal" after she and Jake rent a car to go visit Mark in his foster home. Yet just as my student Adam assumes that the prototypical, ideal American is a white male, Beth has been conditioned to define familial normalcy through the very (white) privilege she criticizes and defines herself against at the beginning of the novel: "a nice car, my husband at my side for once acting like one, my kids in the back, or four out of six, and the fifth coming up, with a boot fulla [sic] picnic food and even a purse with a few bob for extras" (Duff 89). While violence and alcoholism continue to boil beneath the surface, Beth's desire for "the good life"-a life built upon colonial ideals of material security and the naturalized, normalized nuclear, bonded family—could free them from dysfunction. Beth's illusion of "the better life" invites readers to bear witness to the self-deception that colonialism can generate, and how this self-deception can become violent. It further invites readers to speculate about naturalized Western ideals such as individualism, personal success, financial prosperity, and heteronormativity, and to question the extent to which these ideals are sociallyimposed.

Along their way to see Mark, Beth uncovers another layer of her entrapment: her estrangement from Mäori ancestors after marrying Jake and moving to urban Auckland. This estrangement, however, has a long legacy that was passed down to Beth through her own parents. As the Hekes pass through Wainui pa, Beth's hometown, Beth connects her own self-destructive behaviors with those of her parents. Drinking, smoking and neglect killed her mother and could, if she continues on her current trajectory, kill Beth and her 
family. Beth recalls that her "Mum died of [. . .] Lung cancer. From smoking these things, Beth looking frowningly at her cigarette before her body had her sucking at it for what it offered. And love" (Duff 94-95). The closeness of the words "what it offered" (the temporary comforts of smoking) and "love" suggest that she associates this fleeting pleasure with love. Beth's love for "the fist-happy" Jake mirrors her addiction to cigarettes. Both represent love and toxicity to Beth, yet this kind of love is the only one she has learned to recognize, given her mother's example and Mäoris' history as a colonized people. Beth also ponders why her father never expressed love for her mother "because he was of that school of being gruff, tough, manly - manly - and happier when he was around his mates, drinking"- $-\mathrm{a}$ theme that reverberates throughout Beth's upbringing, marriage, and her people's history (95). This story of injury and neglect is not just Beth's or her parents', but the story of many Mäori communities. Moreover, the abusive masculinity embodied in Jake and, by implication, in Beth's father as well, is not simply evidence of affliction in the novel's male characters, but in Mäori men collectively. Beth's self-reflections resonate with college student readers, who are often at a stage in life of questioning their upbringings and family traditions. As Beth tries to make sense of her family history and its relationship to her present condition as an abused woman living in poverty, students can witness the ways in which violence is ingrained and reinforced over decades - an opportunity to consider how their own histories of violence may impact their worldviews.

Toward the end of the novel, traumatized Mäori masculinity turns fatal. Jake rapes his own daughter, Grace, which results in her committing suicide. Told through Grace's perspective, the rape scene induces the disorientation and displacement of rape 
trauma, which invites student-readers to sensorily experience rather than intellectualize the trauma of rape, thereby suspending the inclination to refuse or deny rape testimonies: [T]his voice going, Shhhhhh. And the hand it was stroking her leg. (This is no dream.) [...] The beer stink rose up in wafts to Grace's sense of smell [...] (Is it Dad?) [...] Finger fiddled with her most private part. And a hoarse voice whispered, ya like that, doncha? [...] Rubbing her hair down there. Grunting. Saying shhhh $[\ldots]$ the pain of something inside her. Working its way in [...] Mum? She could hear the faint muffled sound of her mother's distinctive snoring. Mum! in her mind, thinking of mental telepathy, communication between mothers and daughters in trouble without screams or words. Just come, Mum. Oh please come. But daring not to scream $[\ldots]$ And the man breathing his rotten fumes all over her, his whiskers harsh on her face, going: $\mathrm{mmmmm}$, as he kissed her. And she lying there with lips shut tight but daring not to twist away, or even indicate she was awake. And that smell stronger. And kind of knowing what it was ... that it was somehow self-familiar, something sexual, an off-giving, except this sensed as somehow corrupted. By him. This man. Raping her.” (Duff 83-85)

Grace's stream of fractured sensory details discloses what is actually happening. As readers, we come to recognize that what is happening is rape at the same time as Grace does: as she encounters the hoarse voice, a finger and a "something inside her"; as she detects the sounds of her mother's snoring from the next room. Moreover, Grace perceives what rape means in this context: irrevocable corruption. Rape catalyzes recognition; recognition signifies familial abandonment; the abandonment of family, the 
very kinship systems and relationships that provide passage into the world, catalyzes death.

Grace's funeral brings to the fore the colonial undertones of her suicide and of Jake's violence. When the Te Tupaea ${ }^{27}$ recites the whakapapa ${ }^{28}$ Beth's aunt translates: "His ancestry—your ancestry, therefore, Beth, and mine—-he recalls all those tupuna ${ }^{29}$ in the heart of every true Mäori. He is saying, Beth, that we are what we are only because of our past $[\ldots]$ and that we should never forget our past or our future is lost" (Duff 118). In hearing these words, Beth begins to synthesize the many moments of recognition she has throughout the novel. She identifies lack as the core of "what ail[s] her people: lack of knowledge of the past. A history" (ibid). Beth can sense the presence of an absent history within herself, which ultimately compels her to leave Jake, to stop drinking, and to transition from victim/survivor to protector/mother. She exclaims, "Who needs anything else when you got the strength of history supporting ya? I mean to say [. . .] A resurgence of fierce pride, a come-again of a people who Once Were Warriors" (121). This statement underscores cultural belonging as the ultimate antidote to colonial oppression, and quite literally asks student-readers to question the durability of their own support systems.

After Grace's funeral, Beth reclaims the Heke home as a safe haven, kicking Jake out. Instead of attending the funeral, Jake had invited his boozing buddies over. In the film adaptation, Jake consumes so much beer, he exclaims to his buddies, "Have you ever seen so much piss?!," dramatizing alcoholism as a coping mechanism for dealing with trauma. However, Beth refuses the self-destructive cycle she has repeated so many times

\footnotetext{
${ }^{27}$ Te Tupaea is the Mäori word for the chief of a Mäori tribe.

${ }^{28}$ Whakapapa is the Mäori word for a traditional reading of Mäori genealogy at communal ceremonies, such as a funeral.

${ }^{29}$ Tupuna is the Mäori word referring to male ancestry.
} 
with Jake, exclaiming, “The lot of you—out. Jake jumping up and tellin em, Don't listen to her, she's, you know—Beth, c'mon, dear. These're guests. Only reason he was being reasonable because of Grace. Not my guests. Now go. The lot of you" (Duff 140). She then orders Jake to leave, shaming him: “So where was my daughter's father when she was buried? [...] Get out, Jake [ . . ] Get out! [ . . ] And don't you come back, mista [ . . ] Ever" (141). As he leaves, "Beth spat full in his face [. . . standing there, feeling [. . .] feeling almost exultant. Go to hell, mista" (140). Witnessing this scene through Beth's perspective, through her rejection of violence and alcoholism, her ability to see through Jake's "reasonableness" in not beating her for talking back this time, students can experience what reclamation might look like when a victim finally leaves an abusive relationship. They can understand that this reclamation is often hard-won-in Beth's case, as an outcome of her daughter's death—which disrupts the pervasive assumption in a "blame the victim" culture that being abused and getting away from abuse is all about personal choice.

By the end of the novel, Beth returns to the beginning - to the "unkempt, illdirected, neglected" children described on the first page that now inspire her to create a new home that surpasses the superficial, materialistic fantasy she describes on the Heke family outing to visit Mark (Duff 1). In the home Beth returns to at the end of the novel, the Mäori people of Pine Block are still very much on the brink of genocide. Poverty still plagues the community, most everyone living from government check to government check. Yet Beth no longer feels bound to Pine Block's misery. In fact, by staying-more fully inhabiting her home space-Beth finds peace, which is not to be confused with recovery or closure. 
Reading Beth's decision to stay in Pine Block through trauma can challenge assumptions that escaping poverty through upward mobility, a narrative of subscribing to colonial "progress," is ideal. Beth does not flee the site of trauma; rather, she stands within it with greater presence of mind: "Every day; evenings, afternoons when the kids were coming home from school, at nights when they were out on the streets, Beth Heke out there with them. With a message: I'm here to help you. Any of you [. . .] I'll go out onto the streets where all this misery's at and do what I can" (159). In making herself available to the children, Beth claims she will "do [her] best to give you kids your rightful warrior inheritance. Pride in yourself, your poor selves. Not attacking, violent pride but heart pride. Gonna go to my people, my leaders, ask them the way" (161). Beth makes good on her promise, bringing tribal elders to Pine Block to speak to the children about Mäori history_-“The Treaty of Waitangi [ . . ] IT WAS A CONTRACT [ . . ] Which - they - broke" (173). Beth's actions are not a cure or erasure of colonial trauma. Rather, Beth provides an opportunity for the Pine Block community to embrace ragerage directed not at one another but at the trauma of colonization that steals Mäori land, breaks treaties, and forced assimilation. Beth cannot provide this opportunity for her own children, several of them already gone to social welfare (son Mark), gangs (son Nig), and death (daughter Grace). She can, however, advocate for the children of others—of parents lost to alcohol and violence themselves. Beth's transformation can prompt students to envision what community interventions into postcolonial violence might look like. It can prompt students to reconceptualize choice as a return - to family, to place, to a vision of community that is not based on survival, but on history, pride, and amending wrongs of the past. 
Enacting the very project of co-witnessing trauma, of mutually listening for the "truth" of what happened and for the points of resistance and engagement such listening provokes for student-readers, Once Were Warriors exposes both congruencies and contradictions between inside and outside - what is beneath and what is beyond the surface of cultural consciousness about the role colonization has played in Aotearoa New Zealand. The novel is rife with trauma and, while fictional, has had widespread material effects among Mäori New Zealanders. As Kirsten Moana Thompson explains in her essay, “Once Were Warriors: New Zealand's First Indigenous Blockbuster” about Lee Tamahori's film adaptation of Once Were Warriors, through its "harsh unmitigated portrait" of violence in the Mäori community of Pine Block, the film has prompted open, public discussion about the impacts of colonization in New Zealand (234). After the film's 1994 release, a surge of abuse victims fled to shelters, and police noted a marked increase in reports of domestic violence (233). As this phenomenon illustrates, the fictionalized representations of abuse in Once Were Warriors resonate with Mäori people's daily lives, prompting them to acknowledge their traumas as needing and deserving of change.

The violence of Once Were Warriors has also drawn international attention. At the annual Conference for the International Society for the Prevention of Child Abuse and Neglect (ISPCAN) - held in Auckland in 1998, just a few years after the film's release - conference participants and political leaders linked violence in Once Were Warriors with the realities of disproportionate unemployment, imprisonment and violence among Mäori in relation to the Pakeha majority inhabiting New Zealand. In a New Zealand Herald article covering the conference, Member of Parliament Nanaia 
Mahuta attributed such disproportions to " "the reality of the enduring Once Were Warriors lifestyle' and the 'code of silence' in Mäori homes that allow[s] kids to grow up abused and thinking this [is] just part of life" (qtd. in Chateau). The 1998 IPSCAN in Auckland exemplifies how Once Were Warriors effectively creates a language for openly discussing the effects of colonial silencing on Mäori children, as it can facilitate creating a pedagogical language for discussing the effects of co-witnessing trauma. As Once Were Warriors affirms, the "code of silence" erodes Mäori culture, from one generation to the next, and is reinforced by a colonial system that upholds white privilege and denies indigenous opportunity by portraying domestic violence as intrinsic to Mäori identity, rather than as symptomatic of colonial genocide.

The violence embedded in Once Were Warriors and " "the reality of the enduring Once Were Warriors lifestyle" " spotlight deep-seated afflictions between Mäori and Pakeha, colonized and colonizer. Co-witnessing trauma can highlight such imperial fissures, laying a foundation for bringing them into cultural consciousness. In Decolonizing Cultures in the Pacific: Reading, History and Drama in Contemporary Fiction, Susan Y. Najita articulates the importance, in engaging with Mäori literature, of interrogating New Zealand's emerging bicultural nationhood ideology, which promotes shared governance and a decolonial New Zealand. As Najita explains, biculturalism assumes a respectful coexistence. Biculturalism further assumes that Mäori culture is monolithic, subsuming multiple Mäori tribal cultures under the single category of "Mäori." Additionally, biculturalism obscures what Najita calls "the divided-ness [sic] of being both Pakeha and Mäori” (emphasis added, 119). On the one hand, biculturalism represents an effort to link inside and outside - to equalize New Zealand's indigenous 
and settler populations in all facets of social, political life. On the other hand, biculturalism bifurcates inside and outside, presenting an image of equality between Mäori and Pakeha, while concealing an ongoing colonial trend of erasing difference in the name of global capitalism.

Najita argues that trauma plays an active role in shaping the politics and identities of colonized nations, "interven[ing] in the problems of the nation" (19). Trauma is central to the histories of colonized nations, at the same time as those ruling these nations structurally deny that colonial trauma continues to oppress indigenous groups. Literature that deals with issues of postcolonial nation-formation, such as Once Were Warriors, plays a key role in narrating history from the perspective of marginalized groups. The traumas of Once Were Warriors "intervene in the problems of" Aotearoa New Zealand by juxtaposing ideology and reality, biculturalism, and structural inequalities that disproportionately impact Mäori communities—such as the higher rates of poverty among Mäori peoples, which frequently double those among Pakeha peoples, a lower secondary school retention rate for Mäori students, and higher unemployment rates among Mäori youth (Marriott \& Sim). Duff’s novel configures colonization as a key (and ongoing) traumatic event eroding Mäori culture and peoples.

Trauma theory can lead us to understand the misidentification of New Zealand as a bicultural nation. Biculturalism functions as an ideological model of equality for New Zealanders to strive for, but also as a smokescreen over the still-beating heart of colonization, white supremacy: "an institutionally perpetuated system of exploitation and oppression of continents, nations, and people of color by white peoples and nations of the European continent, for the purpose of maintaining and defending a system of wealth, 
power, and privilege" (Martínez 1). By assigning readings about the specific histories of colonized groups; traumatic legacies of colonial trauma, such as internalized oppression; and, neurological and physiological effects of traumatic experience (e.g., as explicated in Bessel A. van der Kolk, M.D.'s The Body Keeps the Score: Brain, Mind, and Body in the Healing of Trauma), a co-witnessing trauma classroom models engaging with literature as a process of personal, cultural, historical reckoning. In addition, trauma theory can lead students to sit with the absence of closure in literature that represents traumatic experience, "producing characters who are unable to work through their impossible histories" (Najita 19). The absence of closure that the characters of Once Were Warriors experience daily materializes in dispossession.

Even as Mäori and Pakeha work together to negotiate land ownership, governance, and issues of cultural autonomy in New Zealand, which might suggest equality, Mäori continue to struggle against the loss of their culture and kinship. As multiple contributors attest in Rethinking Settler Colonialism: History and Memory in Australia, Canada, New Zealand and South Africa, Mäori and Pakeha "coexist uneasily" (Bell 114), their relationship steeped in "unsettled consciousness" (104)—at once "open and chronically controversial" (Thomas 152). Contributor Paul Tapsell explains: "When we walk across our tribal lands we are walking beside our ancestors and using the precedents they have set over the past twenty generations to guide us into the future" (90). When this land is urbanized, as it increasingly is, Mäori tribes lose their ability to connect with their ancestors. Urbanization in Aotearoa New Zealand is a product of the colonial fantasy to bring so-called "civility" to tribal cultures. Quite literally, urbanization replicates and maintains the very conditions of colonization. Industries migrate workers 
to cities, dispersing rural communities; highways increase access for those already mobile, poisoning others with air pollution, such as Pine Block and other governmentowned state housing projects built along highways. Capitalism commodifies daily life, propagating limitless consumption and squeezing out land-based economies.

The Mäori people of Pine Block do not, as bicultural ideology might suggest, experience the benefits of urban growth. They are not nourished and sustained by the land, now built up. Once Were Warriors testifies to this fact, resisting the coercion of closure. The external and interior realms of Pine Block are in a constant state of deprivation and mutually constitute one another's hopelessness. Pine Block is a traumatized community. Its geography dramatizes the instability of "co-existence" implied by biculturalism. An invisible boundary line separates its slum from wealthy Pakeha homes nearby. Tamahori's film adaptation opens with a shot of an idyllic alpine scene on the South Island of New Zealand. Zooming out, the cameras reveal that "the scene" is actually an image on a billboard advertising an electric company, hanging over a part of the highway where Pine Block is located. From the very start of Duff's novel, protagonist Beth Heke voices the pervasive despondence in Pine Block—-her home, a prison. Beth feels inevitably bound to, "A mile-long picture of the same thing [...] just two-storey [sic], side-by-side misery boxes" and "the going-nowhere nobodies who populate this state-owned, half of us state-fed slum" (Duff 1 ). The stark contrast between Beth's poverty and Pakeha wealth consume her, shaping Beth's relationship with her surrounds as one of antagonism and resentment: "Bastard, she'd think, looking out her back window. Lucky white bastard, at that glimpse of two-storey [sic] house through its surround of big old trees and its oh so secure greater surround of rolling green 
pastureland" (ibid). Pine Block's location, skirting the spacious Pakeha homesimpossibly visible, yet just out of reach — is a material testament to the fallacy of biculturalism.

Colonial privilege affords Pine Block's white neighbors physical space as well as mobility in and beyond that space. In contrast, Beth's Mäori body, like her people's Aotearoa, is enslaved by violence while freedom, the fantasy of "a better life," taunts along the periphery. Moreover, Beth's acquiescence to violence — her belief that she is doomed to love her abusive husband Jake forever-illustrates the masterful art of colonial perpetration: teaching the colonized to oppress themselves by strategically replacing their sense of autonomy with complacency or despair. Judith Herman, M.D. defines captivity as prolonged trauma brought about by perpetrators (a) enslaving victims emotionally, mentally, and often physically as well, and (b) persuading victims that they deserve and desire abuse. Fear constructs, in a victim's mind, the perpetrator as "omnipotent" as "his ultimate goal appears to be the creation of a willing victim" (77, 75). Internalized oppression renders Beth and other Mäori vulnerable to continued abuse, compromising their ability to escape. In other words, Beth's "lucky white bastard" statement about the spacious Pakeha homes next door, and her "just can't help myself" rationalization for loving the abusive "Jake the Muss" ${ }^{30}$ indicate that the chronic traumas of poverty and domestic violence have taught her to imprison herself.

The realities of alcoholism, incest, and broken homes in Once Were Warriors can lead students to speculate about how what is within and what is beyond the surface are always-already intersected. Exploring inside and outside in relation to one another can

30 Jake's nickname, "The Muss," is short for muscles and underscores his intimidating physical stature, volatility, and tendency toward violent rages against Beth and others in his immediate and extended Pine Block family. 
disrupt the colonial impulse to divide and conquer; to map territories; to draw and to police geographical borders. Inside signifies both literal and metaphorical "within" spaces: the history of European-Mäori relations contained in textbooks, archives and other historical documents, such as the Treaty of Waitangi; the Heke's and other Mäori families living within the state housing project of Pine Block; the colonial legacy of racialized violence internalized by and enacted amongst the people of Pine Block, such as the incestual rape of Grace by her father; that same legacy internalized by individual characters, like Beth who believes she deserves to be abused. Outside signifies both literal and symbolic "without" spaces: the global circulation of European rule; the wealthy Pakeha living in spacious homes just outside of Pine Block; the colonial desire to maintain global power and control in the form of ongoing violations against indigenous groups, like the Crown's breaches of the Treaty of Waitangi; the loss of Mäori history itself — of connection to ancestors, land, culture, and beliefs greater than "self." In posing the question, "What is the relationship between inside and outside in Once Were Warriors," teachers can ask students to collectively consider how trauma is ultimately an experience that produces an illusion of separation.

Victims of violence like Beth, like the Mäori, incite bystanders "to share the burden of pain [. . . ] demand[ing] action, engagement, and remembering" (Herman 7-8). As teachers, we can teach students to allow trauma to move us, to insist that we witness at the very moment we desire escape, silence, and ignorance; at the very moment we desperately try to just be left alone to go about our own lives. Our proclivity for escapism in the face of catastrophe becomes yet another phenomenon to which we can bear witness, and a co-witnessing trauma classroom can encounter and explore escapism not 
as something to resist or change, but as information. Feeling compelled to escape tells us more about our own comfort zones, especially how traumatic literature can cause us to, at once, reinvest in and break open those comfort zones. Communal witnessing is vital in the process of recognition. Just as survivors require community if they are "to rebuild [a] sense of order and justice" in the world, student-readers require community if they are to recognize and articulate their resistances as such (70). Any refusal to perceive trauma in others - and in ourselves, for that matter - revictimizes survivors, and also impedes healing.

\section{Decolonial Activism in the Classroom}

Bearing traumatic witness can be an act of decolonization, of publically acknowledging and assimilating into cultural consciousness the global mass destruction of indigenous peoples by Europeans and the institutionalization of colonial logics. Students with their own colonized histories and contemporalities may push back against traumatic witnessing in a classroom setting, particularly in classrooms and institutions dominated by white bodies. Likewise, white students who subscribe to colonial logics that minimize or obscure the traumatic legacies of settler colonization may push back against traumatic witnessing, convinced that such work is not (or no longer) necessary. A pedagogy of co-witnessing trauma makes such resistance a productive site of inquiry for making meaning of traumatic events narrated in different texts, and moreover, of the meanings those events generate for student-readers.

The horror of traumatic conditions such as residual colonialism and poverty are made palpable (communicable through the senses) through relationships. As Herman 
explicates, traumatic events "breach the attachments of family, friendship, love, and community. They shatter the construction of the self that is formed and sustained in relation to others. They undermine the belief systems that give meaning to human experience $[\ldots]$ and cast the victim into a state of existential crisis" (51). While Herman's analysis of how trauma impacts relationships is based in a psychiatric/individual conception of health, her analysis is critical in illustrating the trajectory of trauma, or how trauma has more to do with effects and aftermaths than to isolated events or happenings. Restoring these shattered relationships requires not only personal support systems, but also "public acknowledgement of the traumatic event $[\ldots]$ and some form of community action [...] to rebuild the survivor's sense of order and justice" (70). In a classroom setting, collectively bearing witness to traumatic events through literature can function as “public acknowledgement," which can lead to "community action" against colonial injustice. As students collectively sit with the realities of settler colonization's still-open wounds, and as they are asked to routinely articulate their own resistances to and identifications with those wounds, the co-witnessing trauma classroom initiates a restorative process of reconciliation: of confronting the brutality of colonization, while also determining their rights and responsibilities, as university members and global citizens, in transforming traces of colonial tyranny today. As teachers, we can teach reading as an act of decolonial resistance, of defining and redefining the very concept of civility that fueled and fuels colonial rule.

A traumatic literature course modeled on principles of decolonial activism can teach students how reading can become a political act of bearing traumatic witness. Bearing witness to trauma works to counteract colonialism in its myriad forms. This 
chapter's conception of decolonial activism emerges from decolonial theory and principles of activist pedagogy. Broadly understood, decoloniality is an ongoing "thinking and doing" project of dismantling colonial power and restoring sovereignty to colonized territories globally, on the simultaneous levels of institutionalized practices and cultural ideologies (Mignolo 282). Decoloniality works against what semiotician Walter D. Mignolo refers to as "the colonial matrix of power," a paradigm upheld by capitalist imperialism and its exploitation of indigenous labor, resources, and overseas markets for the gain of Euro-American nations (281). While the settlements that historically mark the colonial period have passed, decoloniality suggests, the discrimination and genocide inherent in the colonial machine continue to oppress indigenous peoples globally.

In addition to being an exploitative system, coloniality is also a dominant perception. Feminist philosopher Elizabeth Spelman describes this phenomenon as "boomerang perception," a way of perceiving others through the colonial gaze in which "I look at you and come right back to myself" (12). Boomerang perception, feminist philosopher María Lugones explains, relies upon an understanding of Euro-American white people as original and people of color as imitations of that idealized original (157). Both "racist" and "narcissistic," boomerang perception "denies independence to the seen [and] constructs its object imaginatively as a reflection of the seer. It robs the seen of a separate identity" (156-57). Like Foucault's medical gaze explicated in Chapter Two, the colonial gaze travels in one direction, from colonizer to colonized, reinforcing the seer's sense of omnipotence to survey the unexplored, to control and domesticate the barbarian. No reciprocity exists in or can come from such perception. 
Colonial perception infiltrates into the ways we habitually read literature in academic settings, rendering critical reading an act of mastery (of a text): of asserting positions, of laying claim to particular interpretations to particular readings of a text, of constraining multiplicity, challenging ambiguity, explicating by analyzing and laying claim to particular interpretations to reinforce the reader's own worldviews or images. Traumatic literature classrooms can reverse this kind of boomerang reading in which a reader perceives a text through her $\leftrightarrow$ his own theoretical orientations without recognizing it as such and assuming its "rightness." Listening to the text invites readers to confront their perceptions, experiences, and knowledges, and to examine how they interact with the text, rather than how they interpret the text (a more one-sided relationship). As Stef Craps contends in Postcolonial Witnessing: Trauma Out of Bounds, the field of trauma theory capitulates to colonial perception as the field's founding texts, authored largely by Euro-American theorists, "marginalize or ignore traumatic experiences of non-Western or minority cultures" (2), thereby failing to "take account of the specific social and historical contexts in which trauma narratives are produced and received" (5). Craps suggests that the field can subvert this trend by engaging with "postcolonial literature that bears witness to the suffering engendered by racial or colonial oppression" (5). Taken together, Mignolo's, Spelman's, Lugones', and Craps' contributions to decolonial theory shape this chapter's conception of decolonial as any project of transforming colonial perceptions, relationships, and/or systems globally.

The concept of activism in decolonial activism points to the particular kind of decolonial project that this chapter proposes: collectively bearing witness to literary representations of postcolonial trauma by focusing class activities on interaction, 
collaboration, and community. Bell hooks' foundational feminist pedagogy text, Teaching to Transgress: Education as the Practice of Freedom, links pedagogy and political activism through her concept of "engaged pedagogy." Engaged pedagogy facilitates the building of learning communities, hooks' argues, transgressing the longinstituted banking model of education, Paulo Freire's term from Pedagogy of the Oppressed to describe the predominant model of education in which teachers deposit knowledge into students' brains, which students passively receive and regurgitate (8). For hooks, transgression begins with reconceptualizing learning as an active process. As teachers move beyond "banking education" and toward "mutual engagement," hooks claims, the interactions among teacher(s) and students generate knowledge (205). In a mutually-engaged classroom, knowledge grows exponentially, generating among participants "interest in one another, in hearing one another's voices, in recognizing one another's presence"- not just "the presence of the professor" (8). When teachers and students learn collaboratively, hooks concludes, education transforms students' whole beings. An activist classroom is a collaborative classroom, and collaborative learning is liberation.

In the particular pedagogical context of a traumatic literature course, collaboration is uniquely shaped by trauma. Trauma is a wound - psychic, emotional, physical, spiritual — and the state or condition brought about by that wound. Bounded by language, traumatic events represented in literature conscript student-readers into unfamiliar territory, which can produce silence, catalyze memory, and reveal new ways of communicating about traumatic experience that may have been impossible or unrecognizable before. As Julie Rak posits in “Do Witness: Don't: A Woman's Word and 
Trauma as Pedagogy," traumatic literature beckons students to re-envision themselves as not only readers, but witnesses. By enacting trauma, traumatic literature calls readers "into being as witnesses, a position [students] are not usually asked to occupy" (56). When students have nothing to say in the wake of reading a traumatic text, Rak explains, they may actually be experiencing second-hand trauma or remembering their own traumatic experiences, experiencing a "lack of differentiation between the narrator's experience and the reader's" (60). Their silence may signify a suspended "right to speak that the [student-]witness may feel s/he has at other times" (59). Silence allows readers to "wholly pay attention to the narrative and its affect, so that nothing else matters" (ibid). Sitting with and passing through silence in the classroom, as participants might in hooks' engaged learning community, enables participants to bear witness to trauma through the act of reading with the class and showing up to class with their reading experiences. Teachers and students "become part of a public memory" around traumas represented in the texts, developing "new ways to speak about" those traumas, their own traumas, and the process of witnessing themselves, their peers, and their collective experience as witnesses (62-63). Regardless of whether the territory of traumatic literature invokes silence in a classroom community, however, the collective process of experiencing and interacting with our responses to traumatic literature becomes a critical project of bearing witness. Approached collectively, participants' responses can facilitate the activist work of changing and re-imagining global social life.

This chapter thereby conceptualizes decolonial activism in the classroom as a pedagogical approach to postcolonial literature that emphasizes listening to the text and writing with the text as transformative acts of bearing witness to trauma. Defined by the 
Oxford English Dictionary, activism refers to "the policy of active participation or engagement in a particular sphere of activity; specifically the use of vigorous activity to bring about political or social change" (“Activism”). In its emphasis on "active participation" and "vigorous activity," this definition suggests that activist work can include a wide range of activities and do not limit participants to exclusively highvisibility forms of activism such as rallies, protests and sit-ins - activities that may not be accessible, feasible, desirable, or appropriate in classroom contexts. Bearing traumatic witness is a "vigorous activity [that can] bring about political or social change" in the face of postcolonial traumas (ibid). As students assume positions as reader-witnesses, they must confront their own (mis)perceptions of oppression. As they do, they are faced with the question of how those (mis)perceptions came about; they assess their (mis)perceptions in the context of each other; and from there, determine individualized strategies for intervening in injustices produced by colonial logics. We can see the potential for students to confront their own (mis)perceptions in the film's opening shot, described earlier, in which the knowledge of "what's really being shown here?" is literally a result of expanding perspective.

If trauma were truly an individual pathology, and if traumatic symptoms were comprehensively dysfunctional, where would the purchase for witnessing even be? Traumatic literature ask readers to engage with narrative as a social construction that is not universally therapeutic, necessary, accurate, more factual, or an ideal form of storytelling. Teaching trauma theory in conjunction with Once Were Warriors invites students to engage reading as an act of bearing witness. To read as conscious witnesses, however, we must first learn to identify symptoms of trauma, as we are reading the text, 
and how such symptoms are indicative of colonial trauma. As writing is an act of telling, of bearing witness, reading is an act of listening. Listening through reading necessitates becoming a stranger to what one hears: to subvert the colonial impulse to master, analyze, and interpret; to become estranged from one's prior knowledges, opinions, beliefs, and values; and to dwell in uncertainty. 


\section{CHAPTER VI}

\section{CODA: TRAUMA-A RECONCILIATION}

In closing a dissertation that, at its core, aims to collectivize our understandings of trauma, I present a different discursive formation of trauma from those already showcased: a found poem about reconciling with trauma as a phenomenon that resists classification, and also as one that has catastrophic material dimensions in our daily lives. What follows is a linguistic collage that synthesizes and compiles conclusions this dissertation draws, questions it raises, representations it analyzes, and knowledges it seeks to disrupt — in the service of highlighting, putting into closer conversation, and offering an alternative means of bearing witness to this study as well as to trauma writ large. 


\section{Trauma: A Reconciliation}

Wounding travels

dissolving back into relation

body mind

survivor witness

outside inside-

A tacit form of violence

writing disease into the body

An inevitable impossibility

An occupation of the center

What is the worst thing that has ever happened to you?

A metaphorical alarm clock going off in the brain

Every object touching the hated body, becoming hated

Going from love song to blood bath

A failure to be disturbed

Returning to the disassembled parts:

This one rocks the body to sleep,

This one prepares the body for rape

Loving, knowing arrogance isn't love

Reoccupy silence - listen

Justice demands vulnerability

transforming the pit from prison to encompassing ecosystem

The body, soundless, keeps score

What is the truth of catastrophe? 


\section{REFERENCES}

Abrams, David. Becoming Animal: An Earthly Cosmology. New York: Vintage, 2011.

“Activism.” n.” OED Online. Oxford University Press, June 2015. Web. 10 August 2015.

Alexander, Jeffrey C, Ron Eyerman, Bernard Geisen, Neil J. Smelser, and Piotr Sztompka. Cultural Trauma and Collective Identity. Oakland: University of California Press, 2004.

Anzaldúa, Gloria. Borderlands/La Frontera: The New Mestiza. 4th ed. San Francisco: Aunt Lute Books, 2012.

“American Psychiatric Association DSM-5 Development." Frequently Asked Questions. American Psychiatric Association, 2014. Web. 26 May 2015.

American Psychological Association. Diagnostic and Statistical Manual of Mental Disorders. 5th Ed. Washington, D.C.: American Psychological Association, 2013.

---. Diagnostic and Statistical Manual of Mental Disorders. 4th Ed. Washington, D.C.: American Psychological Association, 1994.

---. Diagnostic and Statistical Manual of Mental Disorders. 3rd Ed. Washington, D.C.: American Psychological Association, 1987.

---. Diagnostic and Statistical Manual of Mental Disorders. 2nd Ed. Washington, D.C.: American Psychological Association, 1968.

---. Diagnostic and Statistical Manual of Mental Disorders. 1st Ed. Washington, D.C.: American Psychological Association, 1952.

Anderegg, David. "What to Say When the Patient Doesn't Talk: Lisbeth Salander and the Problem of Silence." The Psychology of the Girl with the Dragon Tattoo. Eds. Robin S. Rosenberg and Shannon O'Neill. Dallas: Ben Bella Books, 2011. 67-79.

“Aotearoa, New Zealand or Aotearoa New Zealand?" SAYit. UMR Research, 30 May 2013. Web. 27 June 2013. 
“Arousal, n.” OED Online. Oxford University Press, June 2015. Web. 10 August 2015.

“Avoidance, n.” OED Online. Oxford University Press, June 2015. Web. 10 August 2015.

Ballou, Mary and Laura S. Brown. Eds. Rethinking Mental Health \& Disorders: Feminist Perspectives. New York: Guilford Press, 2002.

Barr, Donald A., M.D. Health Disparities in the United States: Social Class, Race, Ethnicity, and Health. Baltimore, MD: Johns Hopkins UP, 2008.

Bell, Leonard. "Auckland's Centerpiece: Unsettled Identities, Unstable Monuments." Rethinking Settler Colonialism: History and Memory in Australia, Canada, Aotearoa New Zealand and South Africa. Ed. Annie E. Coombes. Manchester UP: New York, 2006. 100-20.

Bennett, Jill, and Rosanne Kennedy. Eds. World Memory: Personal Trajectories in Global Time. Houndmills: Palgrave Macmillan, 2003.

Bergen, Doris L. War and Genocide: A Concise History of the Nazi Holocaust. $2^{\text {nd }}$ ed. New York: Rowman \& Littlefield, 2009.

Bergoffen, Debra B. Contesting the Politics of Genocidal Rape: Affirming the Dignity of the Vulnerable Body. Abingdon: Routledge, 2013.

Black, Edwin. War Against the Weak: Eugenics and America's Campaign to Create a Master Race. Westport: Dialog Press, 2012.

Bordwell, David. Figures Traced in Light: On Cinematic Staging. 1st Ed. Oakland: U of California P, 2005.

Buelens, Gert, Sam Durrant, and Robert Eaglestone. Eds. The Future of Trauma Theory: Contemporary Literary and Cultural Criticism. London: Routledge, 2014.

Bureau of Labor Statistics. "Occupational Outlook Handbook: Mental Health Counselors and Marriage and Family Therapists." U.S. Department of Labor. 17 Dec. 2015. Web. 4 May 2016.

Butler, Judith. Precarious Life: The Powers of Mourning and Violence. London: Verso, 2004.

CARA (Communities Against Rape and Abuse). "Taking Risks: Grassroots Community Accountability Strategies." Color of Violence: The INCITE! Anthology. Ed. Incite! Women of Color Against Violence. Cambridge: 
South End Press, 2006. 250-66.

Card, Claudia. "Rape As A Terrorist Institution." Violence, Terrorism, and Justice. Ed. Raymond Gillespie Frey. New York: Cambridge UP, 1991. 296-319.

Carey, Benedict. "Effectiveness of Talk Therapy is Overstated, a Study Says." New York Times. New York Times Company, 30 Sept. 2015. Web. 22 May 2016.

Caruth, Cathy. Listening to Trauma: Conversations with Leaders in the Theory \& Treatment of Catastrophic Experience. Baltimore: Johns Hopkins UP, 2014.

---. Literature in the Ashes of History. Baltimore: Johns Hopkins UP, 2013.

---. Ed. Trauma: Explorations in Memory. Baltimore: Johns Hopkins UP, 1995.

---. Unclaimed Experience: Trauma, Narrative, and History. Baltimore: Johns Hopkins UP, 1996.

Chateau, Carroll du. "The Voice of Youth Wins Forum Hearts and Minds." The New Zealand Herald [Auckland] 08 Sept. 1998, Crime sec.: n. p.

Clarke, Adele E., Laura Mamo, Jennifer Ruth Fosket, Jennifer R. Fishman, and Janet K. Shim. Eds. Biomedicalization: Technoscience, Health, and Illness in the U.S. Durham: Duke U P, 2010.

Collins, Patricia Hill. Black Feminist Thought: Knowledge, Consciousness, and the Politics of Empowerment. $2^{\text {nd }}$ Ed. New York: Routledge, 2000.

Cook, Albert M., and Janice M. Polgar. "Making the Connection." Assistive Technologies (2015): 117-38. The National Child Traumatic Stress Network. Center for Mental Health Services (CMHS), June 2008. Web. 3 July 2015.

Coombes, Annie E. Ed. Rethinking Settler Colonialism: History and Memory in Australia, Canada, Aotearoa New Zealand and South Africa. Manchester UP: New York, 2006.

Craps, Stef. Postcolonial Witnessing: Trauma Out of Bounds. Hampshire, UK: Palgrave Macmillan, 2013.

Cruse, Zoë. "The Forsaken Speciality: Why Do Doctors Look Down on Psychiatry?" Weblog post. Medscape. WebMD LLC, 2015. Web. 19 June 2015. 
Diamond, Stephen A., PhD. "Essential Secrets of Psychotherapy: Repetitive Relationship Patterns." Web log post. Psychology Today. Sussex Publishers, LLC, 14 June 2008. Web. 7 Aug. 2015.

Duff, Alan. Once Were Warriors. New York: Vintage International, 1995.

"Exposure, n." OED Online. Oxford University Press, June 2015. Web. 10 August 2015.

Felman, Shoshana, and Dori Laub, M.D.. Testimony: Crises of Witnessing in Literature, Psychoanalysis, and History. New York: Routledge, 1992.

Florida Center for Instructional Technology. A Teacher's Guide to the Nazi Holocaust. College of Education, University of South Florida, 1997-2013. Web. 17 May 2016.

Foucault, Michel. The Archaeology of Knowledge. New York: Vintage, 1982.

---. The Birth of the Clinic: An Archaeology of Medical Perception. New York: Vintage, 1994.

Frances, Allen, MD. "DSM-5 Is Guide Not Bible-Ignore Its Ten Worst Changes." Web log post. Psychology Today. Sussex Publishers, LLC, 2 Dec. 2012. Web. 18 July 2015.

Frank, Anne. The Diary of A Young Girl. Trans. B. M. Mooyaart. New York: Bantam, 1993.

Freire, Paulo. Pedagogy of the Oppressed. Trans. Myra Bergman Ramos. New York: Continuum, 2000.

Friedman, Matthew J., M.D., Ph.D. PTSD: National Center for PTSD. "PTSD History and Overview: A Brief History of the PTSD Diagnosis." U.S. Department of Veterans Affairs, 25 Mar. 2014. Web. 19 June 2015.

Fry, Melissa S. "Becoming Victims, Becoming Citizens: A Brief History of Gender-Motivated Violence in U.S. Law." Female Victims of Crime: Reality Reconsidered. Eds. Venessa Garcia, and Janice Clifford. Upper Saddle River: Prentice Hall, 2010. 20-44.

Garfield, Rachel L. "Mental Health Financing in the United States: A Primer." The Henry J. Family Foundation. Kaiser Family Foundation, 1 April 2011. Web. 4 May 2016.

“Genocide.” UN News Center. UN, 1996-2013. Web. 23 Nov. 2013.

Girl with the Dragon Tattoo. Dir. David Fincher. Perf. Daniel Craig and Rooney 
Mara. Sony Pictures Entertainment, 2012. DVD.

Girl with the Dragon Tattoo. Dir. Niels Arden Oplev. Perf. Michael Nygvist, Noomi Rapace, and Ewa Froling. Music Box Films, 2010. DVD.

Giroux, Henry. "Paulo Freire and the Politics of Postcolonization." Journal of Advanced Composition 12.1 (1992): 15-26.

Goetz, Christopher G. "Chapter 15: Jean-Martin Charcot And The AnatomoClinical Method Of Neurology." Handbook Of Clinical Neurology 95. History of Neurology (2009): 203-212. ScienceDirect. Web. 19 June 2015.

Gray, Eliza. "The Sexual Assault Crisis On American Campuses." Time.Com (2014): 1. Business Source Complete. Web. 18 June 2014.

Greene, Jeremy A. Prescribing by Numbers: Drugs and the Definition of Disease. Baltimore, MD: Johns Hopkins UP, 2007.

Halifax, Joan, and Marty Peale. "Interbeing: Precepts and Practices of an Applied Ecology." Web log post. Upaya Zen Center. Upaya Zen Center, 18 Nov. 2014. Web. 31 Jan. 2016.

Haraway, Donna. "Manifesto For Cyborgs: Science, Technology, and Socialist Feminism in the 1980s." Socialist Review 80 (1985): 65-108.

Heifferon, Barbara, and Stuart C. Brown. Rhetoric of Healthcare: Essays Toward A New Disciplinary Inquiry. Cresskill, NJ: Hampton P, 2007.

Herman, Judith L. Trauma and Recovery: The Aftermath-From Domestic Abuse to Political Terror. New York: Basic Books, 1997.

Hokowhitu, Brendan. “Tackling Māori Masculinity: A Colonial Genealogy of Savagery and Sport." The Contemporary Pacific 16:2 (Fall 2004). 259-84.

hooks, bell. Teaching to Transgress: Education as the Practice of Freedom. New York: Routledge, 1994.

Ibrahim, Mohamed, and Marina Morrow. "Weaning Off Colonial Psychiatry in Kenya." Colonizing Forces in Psychiatry. Spec. issue of Journal of Ethics in Mental Health 9 (2014): 1-6. Web. 4 May 2016.

INCITE! Women of Color Against Violence. Ed. Color of Violence: The INCITE! Anthology. Cambridge: South End Press, 2006.

Interlandi, Jeneen. "When My Crazy Father Actually Lost His Mind." New York Times Magazine (22 June 2012). Web. 4 May 2016. 
"Intrusion, n." OED Online. Oxford University Press, June 2015. Web. 10 August 2015.

Johnston, Emily R. "Trauma Theory As Activist Pedagogy: Engaging Students As Reader-Witnesses of Colonial Trauma in Once Were Warriors." Special Section on Pedagogy. Spec. issue of Antipodes: A Global Journal of Australian and New Zealand Literature 28.1 (June 2014): 5-17.

Keane, Basil. "Story: Taniwha." Te Ara: The Encyclopedia of New Zealand. Manatū Taonga: New Zealand Ministry for Culture \& Heritage, 4 Dec. 2012. Web. 11 Apr. 2016.

Keating, Christine (Cricket). "Resistant Silences." Silence, Feminism, Power: Reflections at the Edges of Sound. Ed. Sheena Malhotra and Aimee Carrillo Rowe. London: Palgrave Macmillan, 2013.

Kilby, Jane, Richard Crownshaw, and Antony Rowland. Eds. The Future of Memory. New York: Berghahn Books, 2010.

Klaver, Elizabeth, ed. The Body in Medical Culture. New York: SUNY Press, 2009.

Kliff, Sarah. "Seven Facts about America's Mental Health-care System." Web log post. The Washington Post. The Washington Post, 17 Dec. 2012. Web. 10 Aug. 2015.

Koth, Tania L. "How Traumatic Experiences Leave Their Signature on the Genome: An Overview of Epigenetic Pathways in PTSD." Frontiers in Psychiatry 5 (2014): Article 93.

Larsson, Stieg. The Girl With the Dragon Tattoo. New York: Vintage Crime, 2008 .

---. The Girl Who Played With Fire. New York: Vintage Crime, 2008.

---. The Girl Who Kicked the Hornet's Nest. New York: Vintage Crime, 2008.

Laub, Dori. "Truth and Testimony: The Process and the Struggle." Trauma: Explorations in Memory. Ed. Cathy Caruth. Baltimore: Johns Hopkins UP, 1995. 61-75.

Lentin, Ronit. Israel and the Daughters of the Shoah: Reoccupying the Territories of Silence. New York: Berghahn Books, 2000.

Luckhurst, Roger. The Trauma Question. London: Routledge, 2008. 
Lugones, María. Pilgrimages/Peregrinajes: Theorizing Coalition Against Multiple Oppressions. Lanham: Rowman \& Littlefield Publishers, 2003.

"Major Depression Among Adults." NIMH RSS. National Institute of Mental Health, n.d. Web. 20 June 2015.

McCarthy, Lucille Parkinson and Joan Page Gerring. "Revising Psychiatry's Charter Document DSM-IV." Written Communication 11.2 (April 1994): 147-92.

McLeod, Saul. "Maslow's Hierarchy of Needs." Simply Psychology. 2014. Web. 23 May 2016.

Mark, Tami L., Katharine R. Levit, Rita Vandivort-Warren, Jeffrey A. Buck, and Rosanna M. Coffey. "Changes on U.S. Spending on Mental Health and Substance Abuse Treatment, 1986-2005, And Implications for Policy." Health Affairs 30.2 (2011): 284-92.

Marriott, Lisa, and Dalice Sim. "Indicators of Inequality for Māori and Pacific People." Working Papers in Public Finance. Victoria University of Wellington Business School, 2014.

Martínez, Elizabeth. "What is White Supremacy?" SOA Watch: Close the School of the Americas. 2016. Web. 23 May 2016.

Martinsson, Lena, Gabriele Griffin, and Katarina Giritli Nygren, eds. Challenging the Myth of Gender Equality in Sweden. Bristol: Policy Press, 2016.

Mignolo, Walter D. "Geopolitics of Sensing and Knowing: On Decoloniality, Border Thinking, and Epistemic Disobedience." Postcolonial Studies 14.3 (2011): 273-83.

Najita, Susan Y. Decolonizing Cultures in the Pacific: Reading History and Trauma in Contemporary Fiction. New York: Routledge Research in Postcolonial Literatures, 2006.

"Negativism, n." OED Online. Oxford University Press, June 2015. Web. 10 August 2015.

Nussbaum, Abraham M. The Pocket Guide to the DSM-5 Diagnostic Exam. Washington, DC: American Psychiatric Pub., 2013.

Once Were Warriors. Dir. Lee Tamahori. Perf. Rena Owen, Temuera Morrison, Mamaengaroa Kerr-Bell. Fine Line Features, 1995. DVD.

Ortega, Mariana. "Being Lovingly, Knowingly Ignorant: White Feminism and 
Women of Color.” Hypatia 21.3 (2006): 56-74.

O’Toole, Laura L., Jessica R. Schiffman, and Margie L. Kiter Edwards. Eds. Gender Violence: Interdisciplinary Perspectives. 2nd Ed. New York: New York U P, 2007.

Outreach Programme on the Rwanda Genocide and the United Nations. "Background Information on Sexual Violence Used as a Tool of War." Human Rights Documents Online (2016): n. Pg. 2016. Web. 16 May 2016.

Ozick, Cynthia. The Shawl. New York: Vintage International, 1980.

Pollan, Michael. The Botany of Desire: A Plant's-Eye View of the World. New York: Random House Trade Paperbacks, 2002.

Porter, Douglas, M.D. "Colonization by/in Psychiatry: From Over-Medicalization to Democratization." Colonizing Forces in Psychiatry. Spec. issue of Journal of Ethics in Mental Health 9 (2014): 1-7. Web. 4 May 2016.

"Posttraumatic Stress Disorder (PTSD)." American Psychiatric Association DSM5 Development. American Psychiatric Publishing, 2013. Web. 18 June 2014.

"PTSD: National Center for PTSD." The Relationship Between PTSD and Suicide. U.S. Department of Veterans Affairs, 3 Jan. 2014. Web. 10 Aug. 2015.

The Potato Association of America. "Varieties" Potato Association of America: A Professional Society for Advancement of the Potato Industry. Potato Association of America, 2016. Web. 23 May 2016.

Potter, Nancy Nyquist. "Introduction." Colonizing Forces in Psychiatry. Spec. issue of Journal of Ethics in Mental Health 9 (2014): 1. Web. 4 May 2016.

---. 'Reflections on Things We Don't Want to Think About: Intersections of Colonialism, Transgenerational Trauma, and Oppression Within Psychiatry." Colonizing Forces in Psychiatry. Spec. issue of Journal of Ethics in Mental Health 9 (2014): 1-7. Web. 4 May 2016.

Projansky, Sarah. Watching Rape: Film and Television in Postfeminist Culture. New York: New York U P, 2001.

Puar, Jasbir K. "I would rather be a cyborg than a Goddess: BecomingIntersectional in Assemblage Theory." Philosophia 2.1 (2012): 49-66.

Rak, Julie. "Do Witness: Don't: A Woman's Word And Trauma As Pedagogy." 
Topia (York University) 10 (2003): 53-71.

RAINN's Dragon Tattoo Program.” RAINN: Rape, Abuse, Incest National Network. RAINN, 2009. Web. 18 March 2016.

Ratcliffe, Krista. Rhetorical Listening: Identification, Gender, Whiteness. Carbondale: Southern Illinois U P, 2006.

Ringel, Shoshana, and Jerrold R. Brandell. Eds. Trauma: Contemporary Directions in Theory, Practice, and Research. Los Angeles: SAGE Publications, 2012.

Roth, Michael S. Memory, Trauma, and History: Essays on Living with the Past. New York: Columbia UP, 2012.

Rothberg, Michael. "Decolonizing Trauma Studies: A Response." Studies in the Novel 40.1\&2 (2008): 224-34.

Sareen, Jitender et al. "Perceived Barriers to Mental Health Service Utilization in the United States, Ontario, and the Netherlands." Psychiatric Services 58.3 (2007): 357-64.

Saunders, Rebecca. Lamentation and Modernity in Literature, Philosophy, and Culture. New York: Palgrave Macmillan, 2007.

Scarry, Elaine. The Body in Pain: The Making and Unmaking of the World. New York: Oxford UP, 1985.

Segal, Judy Z. Health and the Rhetoric of Medicine. Carbondale: Southern IL UP, 2008.

Semel, Nava. And the Rat Laughed. Victoria, AU: Hybrid Publishers, 2008.

Sherwood, Juanita, Ph.D. "Intergenerational Trauma Isn't Just Another Determinant of Indigenous Peoples' Health." Colonizing Forces in Psychiatry. Spec. issue of Journal of Ethics in Mental Health 9 (2014): 1-7. Web. 4 May 2016.

Shulevitz, Judith. "This is the Science of Survival." The New Republic 245.19 (2014): 86-95.

Smart, Andrew. "A Multidimensional Model of Clinical Utility." International Journal for Quality in Health Care (2006): 1-6.

Spelman, Elizabeth V. Inessential Woman: Problems of Exclusion in Feminist Thought. Boston: Beacon Press, 1988. 
Stein, Nan. "Locating A Secret Problem: Sexual Violence in Elementary and Secondary Schools." Gender Violence: Interdisciplinary Perspectives. 2nd Ed. Eds. Laura L. O’Toole, Jessica R. Schiffman, and Margie L. Kiter Edwards. New York: NYU Press, 2007. 323-32.

Teich, Judith. "Better Data for Better Mental Health Services." Issues in Science and Technology 32.2 (2016): 61-67. Web. 4 May 2016.

Thomas, Nicholas. "The Uses of Captain Cook: Early Exploration in the Public History of Aotearoa New Zealand and Australia." Rethinking Settler Colonialism: History and Memory in Australia, Canada, Aotearoa New Zealand and South Africa. Ed. Annie E. Coombes. Manchester UP: New York, 2006. 140-55.

Thompson, Kirsten Moana. “Once Were Warriors: New Zealand's First Indigenous Blockbuster." Movie Blockbusters. Ed. Julian Stringer. London: Routledge, 2003. 230-41.

van Der Kolk, Bessel A. The Body Keeps the Score: Brain, Mind, and Body in the Healing of Trauma. New York City: Viking, 2014.

van der Kolk, Bessel A., Alexander C. McFarlane, and Lars Weisaeth. Eds. Traumatic Stress: The Effects of Overwhelming Experience on Mind, Body, and Society. New York: Guilford Press, 1996.

Watkins, Mary, and Helene Shulman. Toward Psychologies of Liberation. New York: Palgrave, 2008.

Watters, Ethan. Crazy Like Us: The Globalization Of The American Psyche. United States of America: Free Press, 2010.

“Who Is INCITE?” Incite-national.org. Incite-national.org, 2014. Web. 10 Nov. 2015.

World Health Organization. "Mental Health Financing." Mental Health Policy and Service Guidance Package. 2003. 


\title{
APPENDIX A
}

\section{INFORMED CONSENT LETTER FOR TRAUMA PEDAGOGY STUDY}

\author{
INFORMED CONSENT FOR \\ "Teaching Trauma, Teaching Context: \\ Toward An Activist Pedagogy for Undergraduate Courses with Content on Gender-Based \\ Violence" \\ Dear Potential Participant, \\ Emily Johnston, your instructor for English 128, is conducting a research project to aid in \\ writing her dissertation. The purpose of this research is to investigate teachers' \\ representations of gender-based violence in designing a general education course with \\ this theme, to identify positive and negative reactions to teaching material that deals with \\ gender-based violence, and to develop an activist pedagogy for investigating gender- \\ based violence in a general education course.
}

You are being invited to participate in this research during the time you are enrolled in English 128 (Fall 2013), and participation is completely voluntary. The data gathered during this research will come from the work that is required for this course, which is primarily written assignments (reading quizzes, reading responses and three formal written projects). Emily will also observe and take notes on class discussions.

Participation in this study will not require any additional work on your part; all students in this course will do the same assignments and work regardless of participation in the study.

Risks and Confidentiality: We anticipate few if any risks to you as a participant in this research. You should not feel any pressure from your instructor or peers to participate in this study. Neither will any of your work be identified by name in any public space or publication. Emily will take precautions to protect participants from this risk, including keeping all work confidential; neither your name nor any identifying characteristics will be used in any resulting research or publication.

Your choice whether or not to participate in this study will be kept entirely confidential and will not influence your standing or grade in the course. Emily will not learn the names of the participants until after grades are turned in at the end of the semester. Consent forms will be kept in a file cabinet by the Principle Investigator, Dr. Rebecca Saunders, and unavailable to Emily until that time to ensure that no students receive any inadvertent preferential treatment based on their decision to participate or not participate. 
Benefits: You may benefit from participating in this research project by adding to the construction of knowledge, specifically to the body of knowledge on pedagogy and gender-based violence. You may also benefit from the pedagogics that emerge and are used in this course. Emily may benefit from this research by gathering data to be used in her dissertation in order to earn her doctorate degree. Any resulting publications that utilize this research are likely to further her professional career.

Contacts: If you have any questions or concerns about this research at any time, please contact Emily Johnston, the instructor of this course and Co-Principal Investigator of this study, or Dr. Rebecca Saunders, the Principal Investigator of this study

If you have any questions about your rights as a subject/participant in this research, or if you feel you have been placed at risk, you can contact the Research Ethics \& Compliance Office at:

\author{
Illinois State University \\ Phone: (309) 438-2529 \\ Mailing address: Research Ethics and Compliance Office \\ C/o Office of Research and Sponsored Programs \\ Hovey Hall 310 \\ Campus Box 3040 \\ Normal, IL 61790-3040
}

Please check one of the following boxes:

I choose to participate in this research project

I choose not to participate in this research proj $\square \mathrm{t}$

By signing below, I affirm that I am at least 18 years of age, and I understand that refusal to participate involves no penalty or loss of benefits of any kind and that no there is no penalty to me for withdrawing from this study at any time.

Name (please print)

Date

Signature

Please keep a second copy of this form for your records and thank you for considering this request to participate in this research project. 